Supporting Information

\title{
Total Synthesis of the Putative Structure of Stemonidine: The Definitive Proof of Misassignment
}

Francisco Sánchez-Izquierdo, Pilar Blanco, Félix Busqué, Ramón Alibés, Pedro de March, Marta

Figueredo, ${ }^{*}$ Josep Font, and Teodor Parella ${ }^{\dagger}$

Departament de Química, Universitat Autònoma de Barcelona 08193 Bellaterra, Spain

${ }^{\dagger}$ Servei de Ressonància Magnètica Nuclear, Universitat Autònoma de Barcelona.

marta.figueredo@uab.es

\section{Index}

General Procedures $\quad$ S3

(S)-2-tert-Butyldiphenylsilyloxymethyl-3,4-dihydro-2H-pyrrole 1-oxide (6) S3

Methyl (2S,3R,3aS,6S)-6-(tert-butyldiphenylsilyloxy)methyl-2-(2-methoxycarbonyl)ethylperhydropyrrolo[1,2-b]isoxazole-3-carboxylate (4) and its $(2 R, 3 S, 3 a S, 6 S)$ isomer (11)

Methyl (3S,8S,9R,9aS)-3-(tert-butyldiphenylsilyloxy)methyl-8-hydroxy-5-

oxoperhydro-1 $H$-pyrrolo[1,2-a]azepine-9-carboxylate (3)

Methyl (3S,9aS)-3-(tert-butyldiphenylsilyloxy)methyl-5-oxo-2,3,5,6,7,9a-

hexahydro-1 $H$-pyrrolo[1,2-a]azepine-9-carboxylate (12)

Methyl (3S,8R,9S,9aS)-3-(tert-butyldiphenylsilyloxy)methyl-8,9-dihydroxy-5-

oxooctahydro-1H-pyrrolo[1,2-a]azepine-9-carboxylate (13)

Methyl (3S,8R,9S,9aS)-3-(tert-butyldiphenylsilyloxy)methyl-9-hydroxy-8-

methoxy-5-oxooctahydro- $1 H$-pyrrolo[1,2-a]azepine-9-carboxylate (15)

(3S,8R,9S,9aS)-3-(tert-Butyldiphenylsilyloxy)methyl-9-hydroxy-9-hydroxymethyl-

8-methoxyoctahydro-5H-pyrrolo[1,2-a]azepin-5-one (16)

$(3 S, 8 R, 9 a S)$-3-(tert-Butyldiphenylsilyloxy)methyl-8-methoxytetrahydro- $1 H$ -

pyrrolo[1,2-a]azepine-5,9(6H,9a $H)$-dione (14)

( $\left.2 R, 3^{\prime} S, 8^{\prime} R, 9 \mathrm{a}^{\prime} S\right)$-3'-(tert-Butyldiphenylsilyloxy)methyl-8'-methoxy-4-methylenehexahydro-3 $H$-spiro \{furan-2,9'-pyrrolo[1,2-a]azepin\}-5,5' $\left(4 H, 6^{\prime} H\right)$-dione (18)

( $2 R, 3$ ' $S, 8$ ' $R, 9 \mathrm{a}$ ' $S$ )-3'-Hydroxymethyl-8'-methoxy-4-methylenehexahydro-3Hspiro \{furan-2,9'-pyrrolo[1,2-a]azepine\}-5,5'(4H,6' $H)$-dione (19)

( $2 R, 3$ ' $S, 8$ ' $R, 9 \mathrm{a}$ ' $S$ )-3'-Hydroxymethyl-5,5'-dioxo-8'-methoxy-4-methylenehexahydro-

$3 H$-spiro \{furan-2,9'-pyrrolo[1,2-a]azepine $\}$ - $\left(4 H, 6^{\prime} H\right)$-carbaldehyde (20) 
$\left(2 R, 3^{\prime} S, 8^{\prime} R, 9 \mathrm{a}^{\prime} S, 2^{\prime}{ }^{\prime} R\right)$ - and (2R,3'S,8' $\left.R, 9 \mathrm{a}^{\prime} S\right)-8^{\prime}$ '-Methoxy-4-methylene-3'-[(2S)-4methylene-5-oxotetrahydrofuran-2-yl]hexahydro-3 $H$-spiro \{furan-2,9'-pyrrolo[1,2- $a$ ] azepine $\}-5,5^{\prime}\left(4 H, 6^{\prime} H\right)$-dione (21 and 22)

$\left(2 R, 4 S, 3\right.$ ' $S, 8$ ' $R, 9 \mathrm{a}^{\prime} S, 2^{\prime}$ ' $R, 4$ ' ' $\left.R\right)$ - (23) and ( $2 R, 4 R, 3$ ' $S, 8$ ' $R, 9 \mathrm{a}$ ' $\left.S\right)-8$ '-Methoxy-4-methyl-3'[(2R,4R)-4-methyl-5-oxotetrahydrofuran-2-yl]hexahydro-3 $H$-spiro \{furan-2,9'-pyrrolo[1,2-a]azepine $\}-5\left(4 H, 6^{\prime} H\right)$-one (24)

$\left(2 R, 4 S, 3\right.$ ' $S, 8$ ' $R, 9 \mathrm{a}^{\prime} S, 2$ ' ' $S, 4$ '' $\left.R\right)$ - (25) and (2R,4R,3' $S, 8$ ' $R, 9 \mathrm{a}$ ' $\left.S\right)-8$ '-Methoxy-4-methyl-3'[(2S,4R)-4-methyl-5-oxotetrahydrofuran-2-yl]hexahydro-3H-spiro \{furan-2,9'-

pyrrolo-[1,2-a]azepine $\}-5,5^{\prime}\left(4 H, 6^{\prime} H\right)$-dione (26)

$\left(2 R, 4 R, 3 ' S, 8^{\prime} R, 9 a^{\prime} S\right)-8$ '-Methoxy-4-methyl-3'-[(2S,4S)-4-methyl-5-oxotetrahydrofuran2-yl] hexahydro-3H-spiro \{furan-2,9'-pyrrolo[1,2-a]azepine\}-5(4H,6' $H)$-one (2) and its $(4 S)$ epimer (epi-2)

${ }^{1} \mathrm{H}$ - and ${ }^{13} \mathrm{C}-\mathrm{NMR}$ spectra of compound 6

${ }^{1} \mathrm{H}$ - and ${ }^{13} \mathrm{C}-\mathrm{NMR}$ spectra of compound 4

${ }^{1} \mathrm{H}$ - and ${ }^{13} \mathrm{C}-\mathrm{NMR}$ spectra of compound $\mathbf{3}$

${ }^{1} \mathrm{H}$ - and ${ }^{13} \mathrm{C}-\mathrm{NMR}$ spectra of compound 12 S20

${ }^{1} \mathrm{H}$ - and ${ }^{13} \mathrm{C}-\mathrm{NMR}$ spectra of compound $\mathbf{1 3}$ S21

${ }^{1} \mathrm{H}$ - and ${ }^{13} \mathrm{C}-\mathrm{NMR}$ spectra of compound $\mathbf{1 5}$ S22

${ }^{1} \mathrm{H}$ - and ${ }^{13} \mathrm{C}-\mathrm{NMR}$ spectra of compound $\mathbf{1 6}$ S23

${ }^{1} \mathrm{H}$ - and ${ }^{13} \mathrm{C}-\mathrm{NMR}$ spectra of compound $\mathbf{1 4}$ S24

${ }^{1} \mathrm{H}$ - and ${ }^{13} \mathrm{C}-\mathrm{NMR}$ spectra of compound $\mathbf{1 8}$ S25

${ }^{1} \mathrm{H}$ - and ${ }^{13} \mathrm{C}-\mathrm{NMR}$ spectra of compound 19 S26

${ }^{1} \mathrm{H}$ - and ${ }^{13} \mathrm{C}-\mathrm{NMR}$ spectra of compound 20 S27

${ }^{1} \mathrm{H}$ - and ${ }^{13} \mathrm{C}$-NMR spectra of compound 21 S28

${ }^{1} \mathrm{H}$ - and ${ }^{13} \mathrm{C}$-NMR spectra of compound 22 S29

${ }^{1} \mathrm{H}-\mathrm{NMR}$ spectra of compounds $\mathbf{2 3}$ and $\mathbf{2 4}$ S30

${ }^{1} \mathrm{H}-\mathrm{NMR}$ spectra of compounds $\mathbf{2 5}$ and $\mathbf{2 6}$ S31

${ }^{1} \mathrm{H}$ - and ${ }^{13} \mathrm{C}-\mathrm{NMR}$ spectra of compound 2 S32

${ }^{1} \mathrm{H}$ - and ${ }^{13} \mathrm{C}-\mathrm{NMR}$ spectra of compound epi-2 
General Procedures. Commercially available reagents were used as received. The solvents were dried by distillation over the appropriate drying agents. All reactions were performed avoiding moisture by standard procedures and under nitrogen atmosphere and monitored by analytical thinlayer chromatography (TLC) using silica gel $60 \mathrm{~F}_{254}$ pre-coated aluminum plates $(0.25 \mathrm{~mm}$ thickness). Flash column chromatography was performed using silica gel $60 \AA$, particle size 35-70 $\mu \mathrm{m} .{ }^{1} \mathrm{HNMR}$ spectra were recorded on Bruker DPX250 (250 MHz), Bruker DPX360 (360 MHz), Bruker ARX400 (400 MHz), and Bruker AVANCE500 (500 MHz) spectrometers. Proton chemical shifts are reported in ppm $(\delta)\left(\mathrm{CDCl}_{3} \delta 7.26 \mathrm{ppm}\right) .{ }^{13} \mathrm{CNMR}$ spectra were recorded on Bruker DPX250 (62.5 MHz), Bruker DPX360 (90 MHz), Bruker ARX400 (100 MHz) and Bruker AVANCE500 (125 MHz) spectrometers with complete proton decoupling. Carbon chemical shifts are reported in ppm $(\delta)\left(\mathrm{CDCl}_{3}, \delta 77.0\right)$. NMR signals were assigned with the help of PENDANT, COSY, HSQC, HMBC, and NOESY experiments. Infrared spectra were recorded on a SapphireATR Spectrophotometer; peaks are reported in $\mathrm{cm}^{-1}$. Optical rotation values were measured on an UV-vis Jasco J-175. High resolution mass spectra (HRMS) were recorded at Micromass-AutoSpec using (CI+). Enantiomeric purity of compound 4 was determined by CHPLC on a Waters 2690 chromatograph using a Daicel Chiralpak AD-H 4.6 × 250 mm column ( ${ }^{\mathrm{i}} \mathrm{PrOH} / \mathrm{hexanes,} \mathrm{1/99;} 0.8$ $\left.\mathrm{mL} \cdot \min ^{-1}\right)$.

\section{(S)-2-tert-Butyldiphenylsilyloxymethyl-3,4-dihydro-2H-pyrrole 1-oxide (6)}

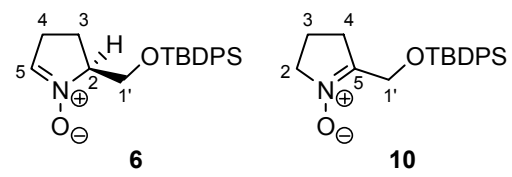

$\mathrm{NaHCO}_{3}(5.70 \mathrm{~g}, 67.9 \mathrm{mmol})$ was added to a stirred solution of amine $9(4.60 \mathrm{~g}, 13.6 \mathrm{mmol})$ in a mixture of acetonitrile-THF $(4: 1,25 \mathrm{~mL})$ and $\mathrm{Na}_{2} \operatorname{EDTA}(0.01 \mathrm{M}, 19 \mathrm{~mL})$. The mixture was then cooled in an ice bath and Oxone ${ }^{\circledR}(8.76 \mathrm{~g}, 14.3 \mathrm{mmol})$ was added portionwise over $2 \mathrm{~h}$. The mixture was stirred at $0{ }^{\circ} \mathrm{C}$ for $20 \mathrm{~min}$ and then diluted with EtOAc $(25 \mathrm{~mL})$. The two phases were separated and the aqueous one was extracted with $\mathrm{CH}_{2} \mathrm{Cl}_{2}(3 \times 40 \mathrm{~mL})$. The combined organic extracts were dried over anhydrous $\mathrm{MgSO}_{4}$ and concentrated under reduced pressure to afford a yellow oil identified as a 1.3:1 mixture of nitrones $\mathbf{6}$ and $\mathbf{1 0}$ (4.30 g, $12.2 \mathrm{mmol}, 90 \%$ yield). The 
ratio 6:10 was determined by ${ }^{1} \mathrm{H}-\mathrm{NMR}$. Pure samples of both nitrones $\mathbf{1 0}$ (first eluted) and $\mathbf{6}$ (second eluted) can be obtained by flash chromatography $\left(\mathrm{CHCl}_{3} / \mathrm{MeOH} 40: 1\right)$.

10: ${ }^{1} \mathbf{H}$ NMR $\left(250 \mathrm{MHz}, \mathrm{CDCl}_{3}\right): \delta 7.62(\mathrm{~m}, 4 \mathrm{H}), 7.38(\mathrm{~m}, 6 \mathrm{H}), 4.67\left(\mathrm{~m}, 2 \mathrm{H}: 2 \mathrm{H}_{1}{ }^{\prime}\right), 3.94(\mathrm{~m}$,

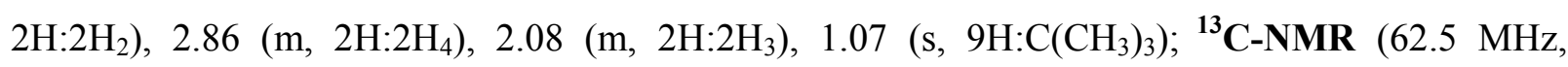
$\left.\mathrm{CDCl}_{3}\right): \delta 148.6\left(\mathrm{C}_{5}\right), 135.4\left(\mathrm{C}_{\mathrm{Ph}}\right), 132.4\left(\mathrm{C}_{\mathrm{Ph}}\right), 129.9\left(\mathrm{C}_{\mathrm{Ph}}\right), 127.7\left(\mathrm{C}_{\mathrm{Ph}}\right), 62.6\left(\mathrm{C}_{1}\right), 59.7\left(\mathrm{C}_{2}\right), 29.5$ $\left(\mathrm{C}_{4}\right), 26.7\left(\mathrm{C}\left(\mathrm{CH}_{3}\right)_{3}\right), 19.1\left(\mathrm{C}\left(\mathrm{CH}_{3}\right)_{3}\right), 17.0\left(\mathrm{C}_{3}\right)$.

6: $[\alpha]_{\mathrm{D}}{ }^{20}=-13\left(c 1.56, \mathrm{CH}_{2} \mathrm{Cl}_{2}\right)$, IR (ATR): 3070, 3048, 2930, 2856, 1587, 1471, 1462, 1427, 1245, 1205, 1111, 1082, 1034, 1009; ${ }^{1} \mathbf{H}$ NMR $\left(250 \mathrm{MHz}, \mathrm{CDCl}_{3}\right): \delta 7.66(\mathrm{~m}, 4 \mathrm{H}), 7.40(\mathrm{~m}, 6 \mathrm{H}), 6.98(\mathrm{bs}$,

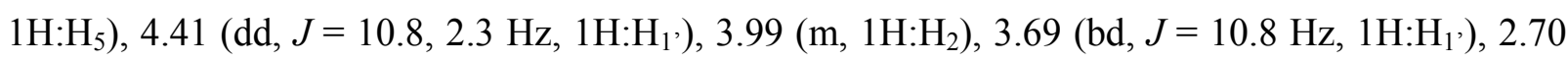
(m, 2H:2H $), 2.33\left(\mathrm{~m}, 2 \mathrm{H}: 2 \mathrm{H}_{3}\right), 1.04\left(\mathrm{~s}, 9 \mathrm{H}: \mathrm{C}_{\left.\left(\mathrm{CH}_{3}\right)_{3}\right)}\right){ }^{13} \mathbf{C}-\mathbf{N M R}\left(62.5 \mathrm{MHz}, \mathrm{CDCl}_{3}\right): \delta 135.5\left(\mathrm{C}_{5}\right)$, $135.3\left(\mathrm{C}_{\mathrm{Ph}}\right), 133.1\left(\mathrm{C}_{\mathrm{Ph}}\right), 132.4\left(\mathrm{C}_{\mathrm{Ph}}\right), 129.6\left(\mathrm{C}_{\mathrm{Ph}}\right), 127.6\left(\mathrm{C}_{\mathrm{Ph}}\right), 73.6\left(\mathrm{C}_{2}\right), 62.0\left(\mathrm{C}_{1}\right), 27.3\left(\mathrm{C}_{4}\right), 26.6$ $\left(\mathrm{C}\left(\mathrm{CH}_{3}\right)_{3}\right), 21.2\left(\mathrm{C}_{3}\right), 19.1\left(C\left(\mathrm{CH}_{3}\right)_{3}\right)$; HRMS $(\mathrm{CI}+)$ : calcd for $\mathrm{C}_{21} \mathrm{H}_{27} \mathrm{NNaO}_{2} \mathrm{Si}: 376.1703[\mathrm{M}+\mathrm{Na}]^{+}$; found 376.1691 .

\section{Methyl (2S,3R,3aS,6S)-6-(tert-butyldiphenylsilyloxy)methyl-2-(2-methoxycarbonyl)ethyl-} perhydropyrrolo[1,2-b]isoxazole-3-carboxylate (4) and its $(2 R, 3 S, 3 \mathrm{a} S, 6 S)$ isomer (11)
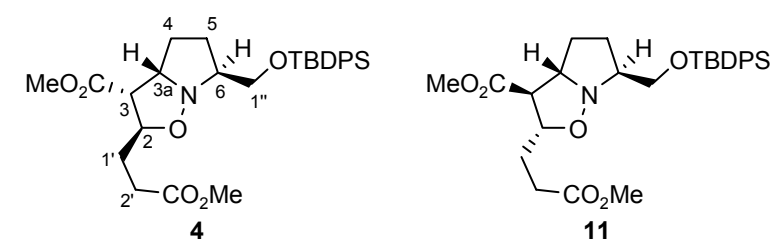

A solution of nitrone $6(1.00 \mathrm{~g}, 2.83 \mathrm{mmol})$ and olefin $5(536 \mathrm{mg}, 3.12 \mathrm{mmol})$ in toluene $(15 \mathrm{~mL})$ was heated at reflux for $10 \mathrm{~h}$. Removal of the solvent and flash chromatography of the crude material (hexanes/EtOAc 4:1) afforded the following fractions: i) recovered 5 (31 $\mathrm{mg}, 0.18 \mathrm{mmol}$ ) as an oil; ii) exo adduct 11 (71 mg, $0.14 \mathrm{mmol}, 5 \%$ yield) as an oil; and iii) endo adduct 4 (1.160 g, $2.21 \mathrm{mmol}, 78 \%$ yield) as an oil.

11: $[\alpha]_{\mathrm{D}}^{20}=-48\left(c 1.60, \mathrm{CH}_{2} \mathrm{Cl}_{2}\right.$ ), IR (ATR): 3071, 3049, 2951, 2931, 2857, 1736, 1461, 1428, 1360, 1262, 1198, 1169, 1110, 1007; ${ }^{1} \mathbf{H}$ NMR $\left(360 \mathrm{MHz}, \mathrm{CDCl}_{3}\right): \delta 7.66(\mathrm{~m}, 4 \mathrm{H}), 7.39(\mathrm{~m}, 6 \mathrm{H})$, 4.13 (dt, $\left.J=8.4,4.0 \mathrm{~Hz}, 1 \mathrm{H}: \mathrm{H}_{2}\right), 3.97$ (q, $J \approx 6.4 \mathrm{~Hz}, 1 \mathrm{H}: \mathrm{H}_{3 \mathrm{a}}$ ), 3.77 (dd, $\left.J=9.8,4.5 \mathrm{~Hz}, 1 \mathrm{H}: \mathrm{H}_{1}{ }^{\prime}\right)$, 3.71 (s, 3H:OCH 3$), 3.66$ (s, 3H:OCH $), 3.54$ (dd, $\left.J=9.8,7.2 \mathrm{~Hz}, 1 \mathrm{H}: \mathrm{H}_{1}{ }^{\prime}\right), 3.47$ (m, 1H:H $), 2.76$ (dd, $\left.J=8.7,5.9 \mathrm{~Hz}, 1 \mathrm{H}: \mathrm{H}_{3}\right), 2.44\left(\mathrm{~m}, 2 \mathrm{H}: 2 \mathrm{H}_{2}\right), 2.13$ (m, 3H: $\left.\mathrm{H}_{4}, \mathrm{H}_{5}, \mathrm{H}_{1^{\prime}}\right), 1.82\left(\mathrm{~m}, 3 \mathrm{H}: \mathrm{H}_{4}, \mathrm{H}_{5}, \mathrm{H}_{1}\right.$ ) , 
1.05 (s, 9H:C(CH$\left.)_{3}\right) ;{ }^{13} \mathbf{C}$ NMR $\left(90 \mathrm{MHz}, \mathrm{CDCl}_{3}\right): \delta 173.4\left(\mathrm{CO}_{2} \mathrm{CH}_{3}\right), 171.6\left(\mathrm{CO}_{2} \mathrm{CH}_{3}\right), 135.6$ $\left(\mathrm{C}_{\mathrm{Ph}}\right), 133.5\left(\mathrm{C}_{\mathrm{Ph}}\right), 133.4\left(\mathrm{C}_{\mathrm{Ph}}\right), 129.6\left(\mathrm{C}_{\mathrm{Ph}}\right), 127.6\left(\mathrm{C}_{\mathrm{Ph}}\right), 80.4\left(\mathrm{C}_{2}\right), 69.4\left(\mathrm{C}_{3 \mathrm{a}}\right), 69.1\left(\mathrm{C}_{6}\right), 65.5\left(\mathrm{C}_{1}{ }^{\prime}\right)$, $58.7\left(\mathrm{C}_{3}\right), 52.2\left(\mathrm{CO}_{2} \mathrm{CH}_{3}\right), 51.6\left(\mathrm{CO}_{2} \mathrm{CH}_{3}\right), 30.6\left(\mathrm{C}_{2}\right), 30.0\left(\mathrm{C}_{4}\right), 28.6\left(\mathrm{C}_{1}\right), 26.9\left(\mathrm{C}_{5}\right), 26.8$

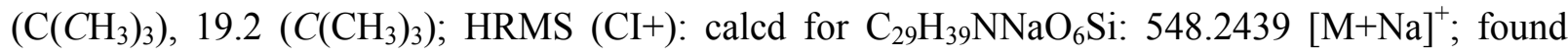
548.2428 .

4: $[\alpha]_{\mathrm{D}}{ }^{20}=-90\left(c\right.$ 2.06, $\left.\mathrm{CH}_{2} \mathrm{Cl}_{2}\right)$;IR (ATR): 3070, 3048, 2951, 2878, 1737, 1429, 1362, 1173, 1112; ${ }^{1}$ H NMR (360 MHz, $\left.\mathrm{CDCl}_{3}\right): \delta 7.66(\mathrm{~m}, 4 \mathrm{H}), 7.42(\mathrm{~m}, 6 \mathrm{H}), 4.18\left(\mathrm{ddd}, J=9.3,8.2,3.8 \mathrm{~Hz}, 1 \mathrm{H}: \mathrm{H}_{2}\right)$, 3.98 (bq, $J \approx 8.1 \mathrm{~Hz}, 1 \mathrm{H}: \mathrm{H}_{3 \mathrm{a}}$ ), 3.86 (dd, $J=10.1,4.8 \mathrm{~Hz}, 1 \mathrm{H}: \mathrm{H}_{1}$ "), 3.70 (s, $3 \mathrm{H}: \mathrm{CO}_{2} \mathrm{CH}_{3}$ ), 3.65 (s, $\left.3 \mathrm{H}: \mathrm{CO}_{2} \mathrm{CH}_{3}\right), 3.60$ (dd, $J=10.1, J=7.3 \mathrm{~Hz}, 1 \mathrm{H}: \mathrm{H}_{1}$ "), $3.25\left(\mathrm{~m}, 1 \mathrm{H}: \mathrm{H}_{6}\right), 3.13$ (t, $\left.J=9.3 \mathrm{~Hz}, 1 \mathrm{H}: \mathrm{H}_{3}\right)$,

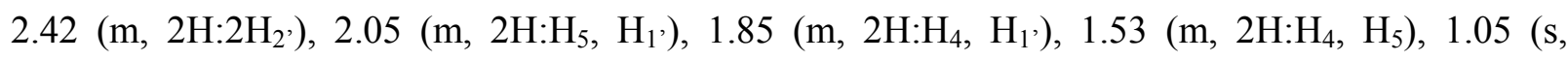
9H:C(CH$\left.)_{3}\right) ;{ }^{13} \mathbf{C}$ NMR (90 MHz, $\left.\mathrm{CDCl}_{3}\right): \delta 173.5\left(\mathrm{CO}_{2} \mathrm{CH}_{3}\right), 170.7\left(\mathrm{CO}_{2} \mathrm{CH}_{3}\right), 135.60\left(\mathrm{C}_{\mathrm{Ph}}\right)$, $135.58\left(\mathrm{C}_{\mathrm{Ph}}\right), 133.7\left(\mathrm{C}_{\mathrm{Ph}}\right), 133.6\left(\mathrm{C}_{\mathrm{Ph}}\right), 129.58\left(\mathrm{C}_{\mathrm{Ph}}\right), 129.56\left(\mathrm{C}_{\mathrm{Ph}}\right), 127.59\left(\mathrm{C}_{\mathrm{Ph}}\right), 127.58\left(\mathrm{C}_{\mathrm{Ph}}\right), 75.4$ $\left(\mathrm{C}_{2}\right), 68.8\left(\mathrm{C}_{6}\right), 66.6\left(\mathrm{C}_{1}{ }^{\prime}\right), 66.2\left(\mathrm{C}_{3 \mathrm{a}}\right), 56.1\left(\mathrm{C}_{3}\right), 51.9\left(\mathrm{CO}_{2} \mathrm{CH}_{3}\right), 51.6\left(\mathrm{CO}_{2} \mathrm{CH}_{3}\right), 30.5\left(\mathrm{C}_{2}\right), 27.34$

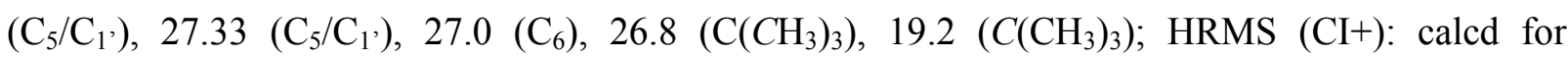
$\mathrm{C}_{29} \mathrm{H}_{39} \mathrm{NNaO}_{6} \mathrm{Si}: 548.2439[\mathrm{M}+\mathrm{Na}]^{+}$; found 548.2424.

Methyl (3S,8S,9R,9aS)-3-(tert-butyldiphenylsilyloxy)methyl-8-hydroxy-5-oxoperhydro-1Hpyrrolo[1,2-a]azepine-9-carboxylate (3)

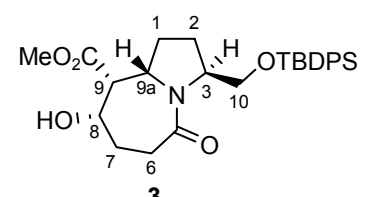

To a solution of 4 (7.95 g, $15.12 \mathrm{mmol})$ in $\mathrm{AcOH}(40 \mathrm{~mL}), \mathrm{Zn}(21.39 \mathrm{~g}, 0.33 \mathrm{~mol})$ was added. After $1.5 \mathrm{~h}$, the reaction mixture was filtered through Celite ${ }^{\circ}$. The organic solvent was evaporated under vacuum. The resulting oil was dissolved in $\mathrm{CHCl}_{3}(30 \mathrm{~mL})$ and washed with aqueous $\mathrm{NH}_{3}$. The aqueous layer was extracted with $\mathrm{CHCl}_{3}(2 \times 15 \mathrm{~mL})$. The combined organic extracts were dried over anhydrous $\mathrm{MgSO}_{4}$ and concentrated. The residue was dissolved in toluene $(40 \mathrm{~mL})$ and the solution was heated at reflux for $16 \mathrm{~h}$. Then, the solution was concentrated in vacuo and the crude product was purified by flash column chromatography on silica gel (EtOAc:hexanes 4:1) to furnish alcohol 3 (6.29 g, 12.69 mmol, 84\% yield): $R_{\mathrm{f}}=0.25$ (EtOAc); $[\alpha]_{\mathrm{D}}{ }^{20}=-57\left(c 0.95, \mathrm{CH}_{2} \mathrm{Cl}_{2}\right)$; IR 
(ATR): 3370, 3070, 3049, 2951, 2931, 2857, 1731, 1620, 1450, 1427, 1391, 1289, 1265, 1107; ${ }^{1} \mathbf{H}$ NMR (360 MHz, $\left.\mathrm{CDCl}_{3}\right): \delta 7.61(\mathrm{~m}, 4 \mathrm{H}), 7.38(\mathrm{~m}, 6 \mathrm{H}), 4.21\left(\mathrm{dt}, J=8.4,4.1 \mathrm{~Hz}, 1 \mathrm{H}: \mathrm{H}_{3}\right), 3.92(\mathrm{~d}, J$ $\left.=9.3 \mathrm{~Hz}, 1 \mathrm{H}: \mathrm{H}_{9 \mathrm{a}}\right), 3.81\left(\mathrm{~m}, 1 \mathrm{H}: \mathrm{H}_{8}\right), 3.77\left(\mathrm{~m}, 2 \mathrm{H}: 2 \mathrm{H}_{10}\right), 3.70\left(\mathrm{~s}, 3 \mathrm{H}: \mathrm{OCH}_{3}\right), 2.96(\mathrm{~d}, J=4.9 \mathrm{~Hz}$,

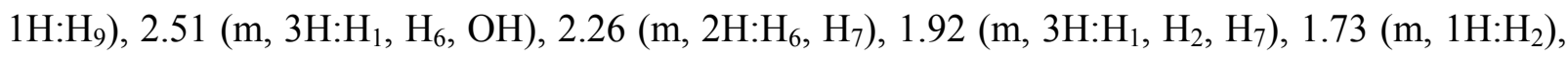
1.04 (s, 9H:C(CH$\left.)_{3}\right) ;{ }^{13} \mathrm{C}$ NMR $\left(90 \mathrm{MHz}, \mathrm{CDCl}_{3}\right): \delta 171.8\left(\mathrm{C}_{5}\right), 171.3\left(\mathrm{CO}_{2} \mathrm{CH}_{3}\right), 135.6\left(\mathrm{C}_{\mathrm{Ph}}\right)$, $135.5\left(\mathrm{C}_{\mathrm{Ph}}\right), 133.7\left(\mathrm{C}_{\mathrm{Ph}}\right), 133.5\left(\mathrm{C}_{\mathrm{Ph}}\right), 129.69\left(\mathrm{C}_{\mathrm{Ph}}\right), 129.65\left(\mathrm{C}_{\mathrm{Ph}}\right), 127.69\left(\mathrm{C}_{\mathrm{Ph}}\right), 127.66\left(\mathrm{C}_{\mathrm{Ph}}\right), 73.8$ $\left(\mathrm{C}_{8}\right), 63.6\left(\mathrm{C}_{10}\right), 60.1\left(\mathrm{C}_{3}\right), 57.0\left(\mathrm{C}_{9 \mathrm{a}}\right), 54.9\left(\mathrm{C}_{9}\right), 52.0\left(\mathrm{OCH}_{3}\right), 33.9\left(\mathrm{C}_{6}\right), 31.7\left(\mathrm{C}_{1}\right), 27.6\left(\mathrm{C}_{7}\right), 26.9$

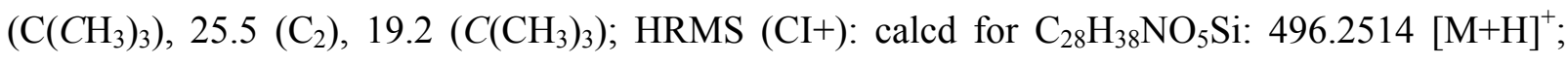
found 496.2512 .

\section{Methyl (3S,9aS)-3-(tert-butyldiphenylsilyloxy)methyl-5-oxo-2,3,5,6,7,9a-hexahydro-1H- pyrrolo[1,2-a]azepine-9-carboxylate (12)}

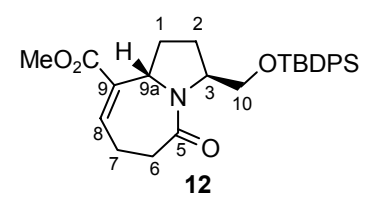

To a solution of alcohol 3 (1.67 g, $3.37 \mathrm{mmol}), \mathrm{PPh}_{3}(1.15 \mathrm{~g}, 4.39 \mathrm{mmol})$, and benzoic acid (0.54 g, $4.39 \mathrm{mmol})$ in anhydrous THF $(30 \mathrm{~mL})$, DIAD $(0.86 \mathrm{~mL}, 4.39 \mathrm{mmol})$ was slowly added. TLC analysis of the reaction mixture (EtOAc) showed that starting 2 was consumed after $1.5 \mathrm{~h}$. The organic solvent was removed under vacuum and the resulting oil was purified by flash column chromatography on silica gel (EtOAc:hexanes 2:1) to afford 12 (1.42 g, $2.97 \mathrm{mmol}, 88 \%$ yield): $R_{\mathrm{f}}$ $=0.58\left(\right.$ EtOAc); $[\alpha]_{\mathrm{D}}{ }^{20}=-159\left(c 0.78, \mathrm{CH}_{2} \mathrm{Cl}_{2}\right)$; IR (ATR): 2931, 2856, 1714, 1644, 1427, 1254, 1216, 1105; ${ }^{1} \mathbf{H}$ NMR $\left(250 \mathrm{MHz}, \mathrm{CDCl}_{3}\right): \delta 7.63(\mathrm{~m}, 4 \mathrm{H}), 7.37(\mathrm{~m}, 6 \mathrm{H}), 6.68\left(\mathrm{~m}, 1 \mathrm{H}: \mathrm{H}_{8}\right), 4.86(\mathrm{~m}$, $\left.1 \mathrm{H}: \mathrm{H}_{9 \mathrm{a}}\right), 4.30\left(\mathrm{~m}, 1 \mathrm{H}: \mathrm{H}_{3}\right), 3.86\left(\mathrm{~m}, 2 \mathrm{H}: 2 \mathrm{H}_{10}\right), 3.73$ (s, 3H: $\left.\mathrm{CO}_{2} \mathrm{CH}_{3}\right), 2.67(\mathrm{qd}, J=13.6,4.4 \mathrm{~Hz}$, $\left.1 \mathrm{H}: \mathrm{H}_{6}\right), 2.48$ (m, 4H: $\left.\mathrm{H}_{1}, \mathrm{H}_{6}, 2 \mathrm{H}_{7}\right), 1.91$ (m, 3H: $\left.\mathrm{H}_{1}, 2 \mathrm{H}_{2}\right), 1.06\left(\mathrm{~s}, 9 \mathrm{H}: \mathrm{C}\left(\mathrm{CH}_{3}\right)_{3}\right) ;{ }^{13} \mathbf{C}$ NMR (62.5 $\left.\mathrm{MHz}, \mathrm{CDCl}_{3}\right): \delta 172.2\left(\mathrm{CO}_{2} \mathrm{CH}_{3}\right), 168.7\left(\mathrm{C}_{5}\right), 139.2\left(\mathrm{C}_{8}\right), 135.5\left(\mathrm{C}_{\mathrm{Ph}}\right), 135.0\left(\mathrm{C}_{9}\right), 133.6\left(\mathrm{C}_{\mathrm{Ph}}\right)$, $133.5\left(\mathrm{C}_{\mathrm{Ph}}\right), 129.6\left(\mathrm{C}_{\mathrm{Ph}}\right), 127.6\left(\mathrm{C}_{\mathrm{Ph}}\right), 63.6\left(\mathrm{C}_{10}\right), 60.2\left(\mathrm{C}_{3}\right), 56.9\left(\mathrm{C}_{9 \mathrm{a}}\right), 51.8\left(\mathrm{CO}_{2} \mathrm{CH}_{3}\right), 34.6\left(\mathrm{C}_{6}\right)$, $31.2\left(\mathrm{C}_{1}\right), 26.9\left(\mathrm{C}_{\left.\left(\mathrm{CH}_{3}\right)_{3}\right),} 25.2\left(\mathrm{C}_{2}\right), 24.7\left(\mathrm{C}_{7}\right), 19.2\left(\mathrm{C}_{\left.\left(\mathrm{CH}_{3}\right)_{3}\right)}\right)\right.$; HRMS $(\mathrm{CI}+)$ : calcd for $\mathrm{C}_{28} \mathrm{H}_{37} \mathrm{NO}_{4} \mathrm{Si}: 478.2408[\mathrm{M}+\mathrm{H}]^{+}$; found, 478.2403. 


\section{Methyl (3S,8R,9S,9aS)-3-(tert-butyldiphenylsilyloxy)methyl-8,9-dihydroxy-5-oxooctahydro-} $1 H$-pyrrolo[1,2-a] azepine-9-carboxylate (13)

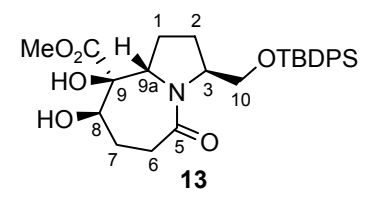

NMO (400 mg, $3.42 \mathrm{mmol})$ and $\mathrm{OsO}_{4}\left(2.5 \%\right.$ in $\left.{ }^{\mathrm{t}} \mathrm{BuOH}, 1.3 \mathrm{~mL}, 0.11 \mathrm{mmol}\right)$ were added to a solution of olefin $12(1.26 \mathrm{~g}, 2.63 \mathrm{mmol})$ in acetone:water $(9: 1,25 \mathrm{~mL})$ at $60{ }^{\circ} \mathrm{C}$. TLC analysis of the reaction mixture showed that starting 12 was consumed after $1.5 \mathrm{~h}$. The reaction mixture was quenched by addition of $\mathrm{NaHSO}_{3}$, the organic solvent was evaporated under vacuum, and the aqueous layer was extracted with $\mathrm{CH}_{2} \mathrm{Cl}_{2}(4 \times 7 \mathrm{~mL})$. The combined organic extracts were dried over anhydrous $\mathrm{MgSO}_{4}$ and concentrated. The yellowish oily residue was purified by flash column chromatography on silica gel to furnish $13(1.24 \mathrm{~g}, 2.42 \mathrm{mmol}, 92 \%$ yield $)$ as a colorless oil: $R_{\mathrm{f}}=$ 0.33 (EtOAc); $[\alpha]_{\mathrm{D}}{ }^{20}=-59$ (c 2.06, $\mathrm{CH}_{2} \mathrm{Cl}_{2}$ ); IR (ATR): 3323, 2930, 2856, 1728, 1616, 1427, 1237 , 1105; ${ }^{1} \mathbf{H}$ NMR $\left(360 \mathrm{MHz}, \mathrm{CDCl}_{3}\right): \delta 7.60(\mathrm{~m}, 4 \mathrm{H}), 7.37(\mathrm{~m}, 6 \mathrm{H}), 4.34\left(\mathrm{~d}, J=8.8 \mathrm{~Hz}, 1 \mathrm{H}: \mathrm{H}_{9 \mathrm{a}}\right)$, 4.09 (m, 1H: $\left.\mathrm{H}_{3}\right), 3.97$ (s, 1H:OH), $3.86\left(\mathrm{~m}, 1 \mathrm{H}: \mathrm{H}_{8}\right), 3.82$ (dd, $\left.J=9.9,3.1 \mathrm{~Hz}, 1 \mathrm{H}: \mathrm{H}_{10}\right), 3.80$ (s, $3 \mathrm{H}:$ $\left.\mathrm{CO}_{2} \mathrm{CH}_{3}\right), 3.73\left(\mathrm{dd}, J=9.9,5.9 \mathrm{~Hz}, 1 \mathrm{H}: \mathrm{H}_{10}\right), 2.95\left(\mathrm{~m}, 2 \mathrm{H}: \mathrm{H}_{6}, \mathrm{OH}\right), 2.21\left(\mathrm{~m}, 3 \mathrm{H}: \mathrm{H}_{1}, \mathrm{H}_{6}, \mathrm{H}_{7}\right), 1.98$ (m, 3H: $\left.\mathrm{H}_{1}, \mathrm{H}_{2}, \mathrm{H}_{7}\right), 1.46$ (m, 1H: $\left.\mathrm{H}_{2}\right), 1.04$ (s, 9H:C(CH$\left.)_{3}\right) ;{ }^{13} \mathbf{C} \mathbf{N M R}\left(90 \mathrm{MHz}, \mathrm{CDCl}_{3}\right): \delta 174.2$ $\left(\mathrm{CO}_{2} \mathrm{CH}_{3}\right), 173.1\left(\mathrm{C}_{5}\right), 135.6\left(\mathrm{C}_{\mathrm{Ph}}\right), 135.56\left(\mathrm{C}_{\mathrm{Ph}}\right), 135.54\left(\mathrm{C}_{\mathrm{Ph}}\right), 133.53\left(\mathrm{C}_{\mathrm{Ph}}\right), 133.51\left(\mathrm{C}_{\mathrm{Ph}}\right), 129.7$ $\left(\mathrm{C}_{\mathrm{Ph}}\right), 127.7\left(\mathrm{C}_{\mathrm{Ph}}\right), 81.7\left(\mathrm{C}_{9}\right), 71.8\left(\mathrm{C}_{8}\right), 63.3\left(\mathrm{C}_{10}\right), 60.0\left(\mathrm{C}_{3}\right), 58.8\left(\mathrm{C}_{9 \mathrm{a}}\right), 53.9\left(\mathrm{CO}_{2} \mathrm{CH}_{3}\right), 30.6\left(\mathrm{C}_{6}\right)$, $26.9\left(\mathrm{C}\left(\mathrm{CH}_{3}\right)_{3}\right), 26.1\left(\mathrm{C}_{7}\right), 25.6\left(\mathrm{C}_{1}\right), 25.0\left(\mathrm{C}_{2}\right), 19.3\left(\mathrm{C}\left(\mathrm{CH}_{3}\right)_{3}\right)$; MS (ESI, MeCN) $m / z 534\left(\mathrm{MNa}^{+}\right)$; Anal. Calcd for $\mathrm{C}_{28} \mathrm{H}_{37} \mathrm{NO}_{6} \mathrm{Si}$ : C, 65.72; H, 7.29; N, 2.74. Found: C, 65.44; H, 7.39; N, 2.66.

Methyl (3S,8R,9S,9aS)-3-(tert-butyldiphenylsilyloxy)methyl-9-hydroxy-8-methoxy-5-oxooctahydro-1H-pyrrolo[1,2-a] azepine-9-carboxylate (15)

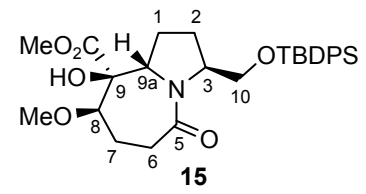

To a solution of diol $13(1.24 \mathrm{~g}, 2.42 \mathrm{mmol})$ in dry THF $(25 \mathrm{~mL})$ at $0{ }^{\circ} \mathrm{C}$, was added $\mathrm{NaH}(291 \mathrm{mg}$, $7.27 \mathrm{mmol})$ in small portions and over $30 \mathrm{~min}$. After stirring $30 \mathrm{~min}$ at $0{ }^{\circ} \mathrm{C}, \mathrm{Me}_{2} \mathrm{SO}_{4}(230 \mu \mathrm{L}, 2.42$ mmol) was added, the reaction mixture was slowly warmed up to room temperature, and then 
stirred overnight. The organic layer was washed with $\mathrm{NH}_{4} \mathrm{Cl}$ (aqueous saturated solution) and the aqueous layer was then extracted with EtOAc $(2 \times 10 \mathrm{~mL})$. The combined organic phases were dried over anhydrous $\mathrm{MgSO}_{4}$ and concentrated under vacuum. Flash column chromatography of the resulting oil on silica gel (EtOAc:hexanes 4:1 to EtOAc) gave starting diol 13 (142 mg, $0.28 \mathrm{mmol}$, $11 \%$ yield) and 15 (940 mg, $1.79 \mathrm{mmol}, 74 \%$ yield, 84\% yield from unrecovered 13 ). 15: $R_{\mathrm{f}}=0.44$ $\left(\right.$ EtOAc); $[\alpha]_{\mathrm{D}}{ }^{20}=-76\left(c 1.95, \mathrm{CH}_{2} \mathrm{Cl}_{2}\right) ;{ }^{1} \mathbf{H}$ NMR $\left(360 \mathrm{MHz}, \mathrm{CDCl}_{3}\right): \delta 7.63(\mathrm{~m}, 4 \mathrm{H}), 7.40(\mathrm{~m}, 6 \mathrm{H})$,

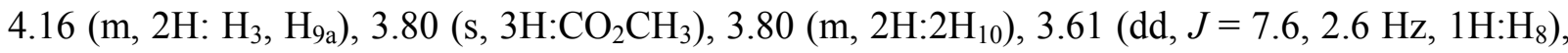
3.54 (s, 1H:OH), 3.37 (s, 3H:OCH $), 2.75$ (ddd, $\left.J=14.4,9.7,6.0 \mathrm{~Hz}, 1 \mathrm{H}: \mathrm{H}_{6}\right), 2.24$ (m, 4H: 2H, $\mathrm{H}_{6}$, $\left.\mathrm{H}_{7}\right), 1.98\left(\mathrm{~m}, 2 \mathrm{H}: \mathrm{H}_{2}, \mathrm{H}_{7}\right), 1.64\left(\mathrm{~m}, 1 \mathrm{H}: \mathrm{H}_{2}\right), 1.06\left(\mathrm{~s}, 9 \mathrm{H}: \mathrm{C}_{\left.\left(\mathrm{CH}_{3}\right)_{3}\right)}\right){ }^{13} \mathbf{C}$ NMR $\left(90 \mathrm{MHz}, \mathrm{CDCl}_{3}\right): \delta$ $172.8\left(\mathrm{CO}_{2} \mathrm{CH}_{3}\right), 171.8\left(\mathrm{C}_{5}\right), 135.5\left(\mathrm{C}_{\mathrm{Ph}}\right), 133.52\left(\mathrm{C}_{\mathrm{Ph}}\right), 133.49\left(\mathrm{C}_{\mathrm{Ph}}\right), 129.6\left(\mathrm{C}_{\mathrm{Ph}}\right), 127.62\left(\mathrm{C}_{\mathrm{Ph}}\right)$, $127.61\left(\mathrm{C}_{\mathrm{Ph}}\right), 81.1\left(\mathrm{C}_{8}\right), 78.0\left(\mathrm{C}_{9}\right), 63.2\left(\mathrm{C}_{10}\right), 61.3\left(\mathrm{C}_{9 \mathrm{a}}\right), 59.9\left(\mathrm{C}_{3}\right), 57.6\left(\mathrm{OCH}_{3}\right), 52.9\left(\mathrm{CO}_{2} \mathrm{CH}_{3}\right)$, $31.3\left(\mathrm{C}_{6}\right), 26.9\left(\mathrm{C}\left(\mathrm{CH}_{3}\right)_{3}\right), 26.1\left(\mathrm{C}_{1}\right), 25.3\left(\mathrm{C}_{2}\right), 20.8\left(\mathrm{C}_{7}\right), 19.2\left(\mathrm{C}_{\left.\left(\mathrm{CH}_{3}\right)_{3}\right)}\right)$; HRMS (CI+): calcd for $\mathrm{C}_{29} \mathrm{H}_{40} \mathrm{NO}_{6} \mathrm{Si}: 526.2619[\mathrm{M}+\mathrm{H}]^{+}$; found 526.2613.

\section{(3S,8R,9S,9aS)-3-(tert-Butyldiphenylsilyloxy)methyl-9-hydroxy-9-hydroxymethyl-8-methoxy- octahydro-5H-pyrrolo[1,2-a] azepin-5-one (16)}

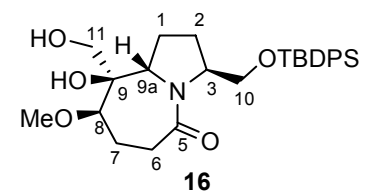

A recently opened solution of $\mathrm{LiBH}_{4}$ in THF $(2 \mathrm{M}, 4.00 \mathrm{~mL}, 8.00 \mathrm{mmol})$ was added to a stirred solution of ester $15(1.05 \mathrm{~g}, 2.00 \mathrm{mmol})$ in dry THF $(20 \mathrm{~mL})$ at room temperature. After $18 \mathrm{~h}$, TLC analysis (EtOAc) of the reaction mixture showed no starting material. The excess of $\mathrm{LiBH}_{4}$ was quenched with $\mathrm{NH}_{4} \mathrm{Cl}$ saturated solution (CAUTION! To avoid a vigorous effervescence, $\mathrm{NH}_{4} \mathrm{Cl}$ must be added very slowly). Then EtOAc was added, and the aqueous phase was extracted with $\mathrm{CH}_{2} \mathrm{Cl}_{2}(2 \times 10 \mathrm{~mL})$. The combined organic extracts were dried over anhydrous $\mathrm{Na}_{2} \mathrm{SO}_{4}$ and concentrated in vacuo. Flash column chromatography purification (EtOAc) afforded 16 (0.965 g, $1.97 \mathrm{mmol}, 97 \%$ yield $): R_{\mathrm{f}}=0.24\left(\right.$ EtOAc); $[\alpha]_{\mathrm{D}}{ }^{20}=-68\left(c 1.32, \mathrm{CH}_{2} \mathrm{Cl}_{2}\right)$; IR (ATR): 3389, 2930, 2856, 1619, 1446, 1426, 1359, 1257, 1173, 1096; ${ }^{1} \mathbf{H}$ NMR $\left(360 \mathrm{MHz}, \mathrm{CDCl}_{3}\right): \delta 7.62$ (m, 4H), 7.39 (m, 6H), 4.19 (m, 2H: $\mathrm{H}_{3}, \mathrm{H}_{9 \mathrm{a}}$ ), 3.79 (dd, $\left.J=9.9,3.2 \mathrm{~Hz}, 1 \mathrm{H}: \mathrm{H}_{10}\right), 3.75$ (dd, $J=9.9,5.6 \mathrm{~Hz}$, $\left.1 \mathrm{H}: \mathrm{H}_{10}\right), 3.71\left(\mathrm{~m}, 1 \mathrm{H}: \mathrm{H}_{8}\right), 3.64$ (d, $\left.J=11.8 \mathrm{~Hz}, 1 \mathrm{H}: \mathrm{H}_{11}\right), 3.45$ (m, $\left.1 \mathrm{H}: \mathrm{H}_{11}\right), 3.39$ (s, 3H:OCH $), 3.21$ 
(d, $J=1.7 \mathrm{~Hz}, 1 \mathrm{H}: \mathrm{OH}), 2.71$ (bt, $\left.J=12.7 \mathrm{~Hz}, 1 \mathrm{H}: \mathrm{H}_{6}\right), 2.32$ (m, 1H: $\left.\mathrm{H}_{2}\right), 2.17$ (m, 3H: $\left.\mathrm{H}_{2}, \mathrm{H}_{6}, \mathrm{H}_{7}\right)$,

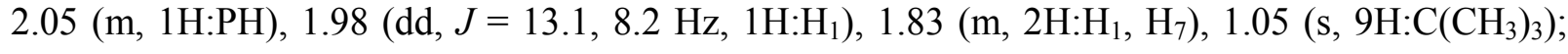

${ }^{13}$ C NMR $\left(90 \mathrm{MHz}, \mathrm{CDCl}_{3}\right): \delta 173.1\left(\mathrm{C}_{5}\right), 135.51\left(\mathrm{C}_{\mathrm{Ph}}\right), 135.49\left(\mathrm{C}_{\mathrm{Ph}}\right), 133.6\left(\mathrm{C}_{\mathrm{Ph}}\right), 133.4\left(\mathrm{C}_{\mathrm{Ph}}\right)$, $129.62\left(\mathrm{C}_{\mathrm{Ph}}\right), 129.58\left(\mathrm{C}_{\mathrm{Ph}}\right), 127.63\left(\mathrm{C}_{\mathrm{Ph}}\right), 127.60\left(\mathrm{C}_{\mathrm{Ph}}\right), 79.3\left(\mathrm{C}_{8}\right), 75.6\left(\mathrm{C}_{9}\right), 63.0\left(\mathrm{C}_{10}\right), 61.3\left(\mathrm{C}_{11}\right)$, $60.1\left(\mathrm{C}_{3}\right), 58.7\left(\mathrm{C}_{9 \mathrm{a}}\right), 56.7\left(\mathrm{OCH}_{3}\right), 29.8\left(\mathrm{C}_{6}\right), 26.9\left(\mathrm{C}\left(\mathrm{CH}_{3}\right)_{3}\right), 26.2\left(\mathrm{C}_{2}\right), 25.3\left(\mathrm{C}_{1}\right), 21.3\left(\mathrm{C}_{7}\right), 19.2$ $\left(C\left(\mathrm{CH}_{3}\right)_{3}\right)$; HRMS (CI+): calcd for $\mathrm{C}_{28} \mathrm{H}_{40} \mathrm{NO}_{5} \mathrm{Si}: 498.2670[\mathrm{M}+\mathrm{H}]^{+}$; found 498.2671.

\section{(3S,8R,9aS)-3-(tert-Butyldiphenylsilyloxy)methyl-8-methoxytetrahydro-1H-pyrrolo[1,2-a]az- epine-5,9(6H,9aH)-dione (14)}

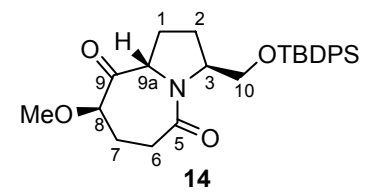

A solution of diol $16(1.28 \mathrm{~g}, 2.56 \mathrm{mmol})$ in dry THF $(25 \mathrm{~mL})$ was added to stirred suspension of $\mathrm{Pb}(\mathrm{OAc})_{4}(1.71 \mathrm{~g}, 3.85 \mathrm{mmol})$ in dry THF $(38 \mathrm{~mL})$ at room temperature. After $1.5 \mathrm{~h}$ TLC analysis (EtOAc) of the reaction mixture revealed the absence of diol 16. The mixture was filtered through Celite ${ }^{\circledR}$, the organic solvent was removed under vacuum and the resulting colorless oil was purified by flash column chromatography on silica gel (EtOAc:hexanes 1:1) to give ketone $14(1.09 \mathrm{~g}, 2.35$ mmol, 92\% yield): $R_{\mathrm{f}}=0.52$ (EtOAc); $[\alpha]_{\mathrm{D}}{ }^{20}=-81\left(c 1.35, \mathrm{CH}_{2} \mathrm{Cl}_{2}\right)$; IR (ATR): 2931, 2857, 1731, 1620, 1427, 1189, 1105; ${ }^{1} \mathbf{H}$ NMR (360 MHz, CDCl $): \delta 7.61(\mathrm{~m}, 4 \mathrm{H}), 7.35$ (m, 6H), 4.43 (dd, $J=$ 8.7, $\left.3.1 \mathrm{~Hz}, 1 \mathrm{H}: \mathrm{H}_{9 \mathrm{a}}\right), 4.27\left(\mathrm{~m}, 1 \mathrm{H}: \mathrm{H}_{3}\right), 4.04\left(\mathrm{dd}, J=8.1,6.5 \mathrm{~Hz}, 1 \mathrm{H}: \mathrm{H}_{8}\right), 3.90$ (dd, $J=10.2,5.2 \mathrm{~Hz}$, $\left.1 \mathrm{H}: \mathrm{H}_{10}\right), 3.78$ (dd, $\left.J=10.2,2.8 \mathrm{~Hz}, 1 \mathrm{H}: \mathrm{H}_{10}\right), 3.31$ (s, 3H:OCH$), 2.60\left(\mathrm{~m}, 1 \mathrm{H}: \mathrm{H}_{6}\right), 2.36\left(\mathrm{~m}, 3 \mathrm{H}: \mathrm{H}_{1}\right.$,

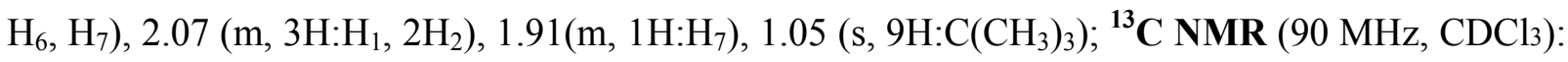
$\delta 208.4\left(\mathrm{C}_{9}\right), 170.9\left(\mathrm{C}_{5}\right), 135.51\left(\mathrm{C}_{\mathrm{Ph}}\right), 135.48\left(\mathrm{C}_{\mathrm{Ph}}\right), 133.3\left(\mathrm{C}_{\mathrm{Ph}}\right), 133.2\left(\mathrm{C}_{\mathrm{Ph}}\right), 129.7\left(\mathrm{C}_{\mathrm{Ph}}\right), 127.7$ $\left(\mathrm{C}_{\mathrm{Ph}}\right), 82.5\left(\mathrm{C}_{8}\right), 64.0\left(\mathrm{C}_{9 \mathrm{a}}\right), 63.4\left(\mathrm{C}_{10}\right), 59.5\left(\mathrm{C}_{3}\right), 57.7\left(\mathrm{OCH}_{3}\right), 31.7\left(\mathrm{C}_{6}\right), 28.6\left(\mathrm{C}_{1}\right), 28.2\left(\mathrm{C}_{7}\right), 26.9$ $\left(\mathrm{C}\left(\mathrm{CH}_{3}\right)_{3}\right)$, $26.6\left(\mathrm{C}_{2}\right), 19.2\left(\mathrm{C}_{\left.\left(\mathrm{CH}_{3}\right)_{3}\right)}\right)$ HRMS $(\mathrm{CI}+)$ : calcd for $\mathrm{C}_{27} \mathrm{H}_{36} \mathrm{NO}_{4} \mathrm{Si}: 466.2408[\mathrm{M}+\mathrm{H}]^{+}$; found 466.2404 . 
(2R,3'S,8'R,9a'S)-3'-(tert-Butyldiphenylsilyloxy)methyl-8'-methoxy-4-methylenehexahydro$3 H$-spiro\{furan-2,9'-pyrrolo[1,2-a] azepin\}-5,5'(4H,6' $H)$-dione (18)

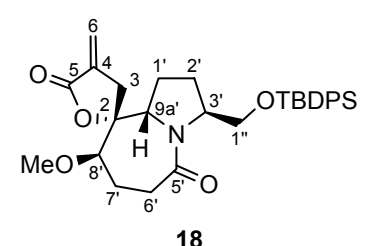

To a stirred solution of ketone $14(1.09 \mathrm{~g}, 2.35 \mathrm{mmol})$ in dry THF $(25 \mathrm{~mL})$ under $\mathrm{N}_{2}$ atmosphere, Zn (460 mg, $7.04 \mathrm{mmol}$ ) was added. The mixture was heated up and, when reflux started, a solution of ethyl 2-(bromomethyl)acrylate, 17, (486 $\mu \mathrm{L}, 3.52 \mathrm{mmol})$ in dry THF $(12 \mathrm{~mL})$ was added. After $1 \mathrm{~h}$ of heating at reflux, TLC analysis $\left(\mathrm{Et}_{2} \mathrm{O}\right)$ revealed the absence of $\mathbf{1 4}$ in the reaction mixture. The mixture was filtered through Celite ${ }^{\circledR}$ and the organic solvent evaporated under vacuum. The remaining colorless oil was dissolved in $\mathrm{CH}_{2} \mathrm{Cl}_{2}(10 \mathrm{~mL})$ and washed with $\mathrm{NH}_{4} \mathrm{Cl}$ saturated solution. The aqueous phase was extracted with $\mathrm{CH}_{2} \mathrm{Cl}_{2}(2 \times 5 \mathrm{~mL})$. The combined organic layers were dried over anhydrous $\mathrm{MgSO}_{4}$ and concentrated in vacuo. Purification of the crude material by flash column chromatography on silica gel $\left(\mathrm{Et}_{2} \mathrm{O}\right)$ furnished lactone $\mathbf{1 8}(1.08 \mathrm{~g}$, $2.01 \mathrm{mmol}, 86 \%$ yield $): R_{\mathrm{f}}=0.18\left(\mathrm{Et}_{2} \mathrm{O}\right) ;[\alpha]_{\mathrm{D}}{ }^{20}=-46\left(c 0.5, \mathrm{CH}_{2} \mathrm{Cl}_{2}\right) ;{ }^{1} \mathbf{H}$ NMR $(360 \mathrm{MHz}$, $\left.\mathrm{CDCl}_{3}\right): \delta 7.60(\mathrm{~m}, 4 \mathrm{H}), 7.38(\mathrm{~m}, 6 \mathrm{H}), 6.26\left(\mathrm{t}, J=2.9 \mathrm{~Hz}, 1 \mathrm{H}: \mathrm{H}_{6}\right), 5.64\left(\mathrm{t}, J=2.5 \mathrm{~Hz}, 1 \mathrm{H}: \mathrm{H}_{6}\right), 4.26$ $\left(\mathrm{m}, 1 \mathrm{H}: \mathrm{H}_{3^{\prime}}\right), 4.02\left(\mathrm{~d}, J=9.0 \mathrm{~Hz}, 1 \mathrm{H}: \mathrm{H}_{9 \mathrm{a}^{\prime}}\right), 3.87$ (dd, $\left.J=10.0,5.3 \mathrm{~Hz}, 1 \mathrm{H}: \mathrm{H}_{1}{ }^{\prime}\right), 3.80(\mathrm{dd}, J=10.0$, $3.0 \mathrm{~Hz}, 1 \mathrm{H}: \mathrm{H}_{1}$ ), 3.34 (s, 3H:OCH${ }_{3}$ ), 3.30 (dd, $\left.J=8.2,2.7 \mathrm{~Hz}, 1 \mathrm{H}: \mathrm{H}_{8^{\prime}}\right), 2.94$ (dt, $J=18.1,2.8 \mathrm{~Hz}$,

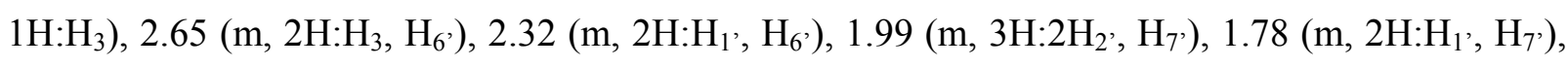
1.04 (s, 9H:C(CH$\left.)_{3}\right) ;{ }^{13} \mathbf{C}$ NMR $\left(90 \mathrm{MHz}, \mathrm{CDCl}_{3}\right): \delta 171.4\left(\mathrm{C}_{5}\right), 169.4\left(\mathrm{C}_{5}\right), 135.53\left(\mathrm{C}_{\mathrm{Ph}}\right), 135.51$ $\left(\mathrm{C}_{\mathrm{Ph}}\right), 134.4\left(\mathrm{C}_{4}\right), 133.6\left(\mathrm{C}_{\mathrm{Ph}}\right), 133.5\left(\mathrm{C}_{\mathrm{Ph}}\right), 129.7\left(\mathrm{C}_{\mathrm{Ph}}\right), 129.6\left(\mathrm{C}_{\mathrm{Ph}}\right), 127.7\left(\mathrm{C}_{\mathrm{Ph}}\right), 127.6\left(\mathrm{C}_{\mathrm{Ph}}\right), 122.2$ $\left(\mathrm{C}_{6}\right), 85.4\left(\mathrm{C}_{2}\right), 82.8\left(\mathrm{C}_{8^{\prime}}\right), 63.3\left(\mathrm{C}_{1}{ }^{\prime}\right), 61.1\left(\mathrm{C}_{9 \mathrm{a}^{\prime}}\right), 59.9\left(\mathrm{C}_{3^{\prime}}\right), 57.6\left(\mathrm{OCH}_{3}\right), 34.5\left(\mathrm{C}_{3}\right), 30.8\left(\mathrm{C}_{6^{\prime}}\right)$, $26.9\left(\mathrm{C}\left(\mathrm{CH}_{3}\right)_{3}\right), 26.4\left(\mathrm{C}_{2}\right), 26.1\left(\mathrm{C}_{1}{ }^{\prime}\right), 20.6\left(\mathrm{C}_{7^{\prime}}\right), 19.3\left(\mathrm{C}_{\left.\left(\mathrm{CH}_{3}\right)_{3}\right)}\right)$; HRMS (CI+): calcd for $\mathrm{C}_{31} \mathrm{H}_{40} \mathrm{NO}_{5} \mathrm{Si}: 534.2670[\mathrm{M}+\mathrm{H}]^{+}$; found 534.2668. 
$\left(2 R, 3 ' S, 8\right.$ ' $\left.R, 9 a^{\prime} S\right)-3$ '-Hydroxymethyl-8'-methoxy-4-methylenehexahydro-3H-spiro\{furan-2,9'pyrrolo[1,2-a]azepine $\}-5,5$ ' $\left(4 H, 6^{\prime} H\right)$-dione (19)

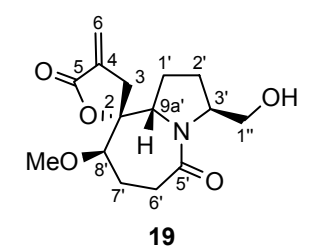

A solution of $\mathrm{Et}_{3} \mathrm{~N} \cdot 3 \mathrm{HF}(6.6 \mathrm{~mL}, 40.3 \mathrm{mmol})$ was added to a stirred solution of $\mathbf{1 8}(1.08 \mathrm{~g}, 2.01$ $\mathrm{mmol})$ in dry THF $(34 \mathrm{~mL})$. The reaction mixture was heated at the reflux temperature for $4 \mathrm{~h}$, when TLC analysis showed no starting material. The solution was concentrated under vacuum and the resulting oil was purified by flash column chromatography on silica gel (EtOAc to EtOAc:EtOH 1:1) to furnish alcohol 19 (515 mg, $1.74 \mathrm{mmol}, 87 \%$ yield): $[\alpha]_{\mathrm{D}}{ }^{20}=-115\left(c 0.22\right.$, EtOH); ${ }^{1} \mathbf{H} \mathbf{~ N M R}$ $\left(360 \mathrm{MHz}, \mathrm{CDCl}_{3}\right): \delta 6.26\left(\mathrm{t}, J=3.0 \mathrm{~Hz}, 1 \mathrm{H}: \mathrm{H}_{6}\right), 5.67\left(\mathrm{t}, J=2.6 \mathrm{~Hz}, 1 \mathrm{H}: \mathrm{H}_{6}\right), 4.55$ (m, 1H:OH),

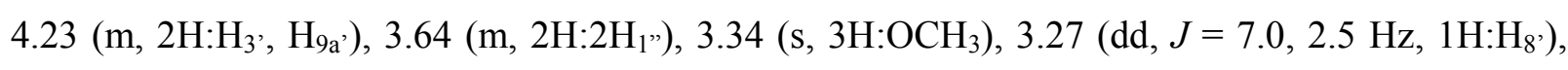
$2.92\left(\mathrm{dt}, J=18.3,2.9 \mathrm{~Hz}, 1 \mathrm{H}: \mathrm{H}_{3}\right), 2.80$ (ddd, $\left.J=15.0,10.5,5.0 \mathrm{~Hz}, 1 \mathrm{H}: \mathrm{H}_{6}\right), 2.73$ (dt, , $J=18.3$, $\left.2.5 \mathrm{~Hz}, 1 \mathrm{H}^{\mathrm{H}} \mathrm{H}_{3}\right), 2.39$ (ddd, $J=14.4,5.8,4.9 \mathrm{~Hz}, 1 \mathrm{H}: \mathrm{H}_{6}$ ), $2.02\left(\mathrm{~m}, 4 \mathrm{H}: \mathrm{H}_{1}, \mathrm{H}_{2}, 2 \mathrm{H}_{7}\right.$ ), 1.77 (m, 1H: $\left.\mathrm{H}_{1}{ }^{\prime}\right), 1.56\left(\mathrm{~m}, 1 \mathrm{H}: \mathrm{H}_{2}{ }^{\prime}\right) ;{ }^{13} \mathbf{C}$ NMR (90 MHz, $\left.\mathrm{CDCl}_{3}\right)$ : $\delta 174.2\left(\mathrm{C}_{5}\right), 169.2\left(\mathrm{C}_{5}\right), 133.9\left(\mathrm{C}_{4}\right), 122.9$ $\left(\mathrm{C}_{6}\right), 84.3\left(\mathrm{C}_{2}\right), 82.5\left(\mathrm{C}_{8^{\prime}}\right), 66.7\left(\mathrm{C}_{1^{\prime}}\right), 62.5\left(\mathrm{C}_{3}{ }^{\prime}\right), 61.0\left(\mathrm{C}_{9 \mathrm{a}}{ }^{\prime}\right), 57.4\left(\mathrm{OCH}_{3}\right), 35.0\left(\mathrm{C}_{3}\right), 30.5\left(\mathrm{C}_{6}{ }^{\prime}\right)$, $27.3\left(\mathrm{C}_{2}\right), 26.0\left(\mathrm{C}_{1}\right), 20.5\left(\mathrm{C}_{7}\right)$; HRMS $(\mathrm{CI}+)$ : calcd for $\mathrm{C}_{15} \mathrm{H}_{22} \mathrm{NO}_{5}: 296.1492[\mathrm{M}+\mathrm{H}]^{+}$; found 296.1497.

(2R,3'S,8' $\left.R, 9 a^{\prime} S\right)-3$ '-Hydroxymethyl-5,5'-dioxo-8'-methoxy-4-methylenehexahydro-3Hspiro\{furan-2,9'-pyrrolo[1,2-a] azepine $\}$-(4H,6' $H)$-carbaldehyde (20)

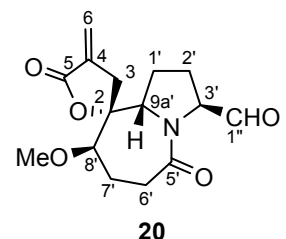

A commercially available solution of the Dess-Martin periodinane in $\mathrm{CH}_{2} \mathrm{Cl}_{2}(15 \% \mathrm{wt}, 1.85 \mathrm{~mL}$, $0.89 \mathrm{mmol}$ ) was added via syringe to a solution of alcohol 19 (219 $\mathrm{mg}, 0.74 \mathrm{mmol})$ in dry $\mathrm{CH}_{2} \mathrm{Cl}_{2}$ $(6.1 \mathrm{~mL})$. After $2 \mathrm{~h}$ of stirring at room temperature, TLC analysis indicates the complete consumption of the starting material. The solution was washed with $\mathrm{NaHCO}_{3}$ saturated solution 
containing a sevenfold excess of $\mathrm{Na}_{2} \mathrm{~S}_{2} \mathrm{O}_{3}$, the aqueous phase was extracted with $\mathrm{CH}_{2} \mathrm{Cl}_{2}(2 \times 5 \mathrm{~mL})$, the combined organic extracts were dried over anhydrous $\mathrm{Na}_{2} \mathrm{SO}_{4}$ and concentrated in vacuo. Flash column chromatography on silica gel (EtOAc) of the resulting oil provided aldehyde 20 (200 mg, 0.68 mmol, 92\% yield): $R_{\mathrm{f}} 0.43$ (EtOAc: $\left.\mathrm{MeOH} 9: 1\right) ;[\alpha]_{\mathrm{D}}{ }^{20}=-149\left(c 0.65, \mathrm{CH}_{2} \mathrm{Cl}_{2}\right) ;{ }^{1} \mathbf{H}$ NMR (500 $\left.\mathrm{MHz}, \mathrm{CDCl}_{3}\right): \delta 9.54\left(\mathrm{~d}, J=1.5 \mathrm{~Hz}, 1 \mathrm{H}: \mathrm{H}_{1}{ }^{\prime}\right), 6.30\left(\mathrm{t}, J=3.0 \mathrm{~Hz}, 1 \mathrm{H}: \mathrm{H}_{6}\right), 5.71(\mathrm{t}, J=2.6 \mathrm{~Hz}$, $\left.1 \mathrm{H}: \mathrm{H}_{6}\right), 4.58\left(\mathrm{~m}, 1 \mathrm{H}: \mathrm{H}_{3}\right)$ ), 4.34 (bd, $\left.J=8.24 \mathrm{~Hz}, 1 \mathrm{H}: \mathrm{H}_{9 \mathrm{a}^{\prime}}\right), 3.37$ (s, 3H:OCH$), 3.33$ (dd, $J=6.3,2.4$ Hz, 1H: $\mathrm{H}_{8}$ ), 2.96 (dt, $\left.J=18.4,2.9 \mathrm{~Hz}, 1 \mathrm{H}: \mathrm{H}_{3}\right), 2.88$ (ddd, $J=14.9,10.5,4.5 \mathrm{~Hz}, 1 \mathrm{H}: \mathrm{H}_{6}$ ), 2.76 (bd, $\left.J=18.4 \mathrm{~Hz}, 1 \mathrm{H}: \mathrm{H}_{3}\right), 2.40\left(\mathrm{~m}, 1 \mathrm{H}: \mathrm{H}_{6}\right), 2.04\left(\mathrm{~m}, 4 \mathrm{H}: \mathrm{H}_{1}, \mathrm{H}_{2}, 2 \mathrm{H}_{7^{\prime}}\right), 1.85$ (m, 2H: $\mathrm{H}_{1}, \mathrm{H}_{2}$ ) $){ }^{13} \mathbf{C} \mathbf{N M R}$ (125 MHz, $\left.\mathrm{CDCl}_{3}\right): \delta 198.1\left(\mathrm{C}_{1}{ }^{\prime}\right), 172.5\left(\mathrm{C}_{5}{ }^{\prime}\right), 169.1\left(\mathrm{C}_{5}\right), 133.7\left(\mathrm{C}_{4}\right), 123.4\left(\mathrm{C}_{6}\right), 83.7\left(\mathrm{C}_{2}\right), 82.5$ $\left(\mathrm{C}_{8^{\prime}}\right), 66.1\left(\mathrm{C}_{3^{\prime}}\right), 59.8\left(\mathrm{C}_{9 \mathrm{a}}\right), 57.3\left(\mathrm{OCH}_{3}\right), 35.2\left(\mathrm{C}_{3}\right), 29.2\left(\mathrm{C}_{6}\right), 26.2 / 24.1\left(\mathrm{C}_{1}, \mathrm{C}_{2}\right), 20.7\left(\mathrm{C}_{7^{\prime}}\right)$; HRMS (CI+): calcd for $\mathrm{C}_{15} \mathrm{H}_{20} \mathrm{NO}_{5}: 294.1336[\mathrm{M}+\mathrm{H}]^{+}$; found 294.1334 .

$\left(2 R, 3 ' S, 8 ' R, 9 a^{\prime} S, 2\right.$ '' $\left.R\right)$ - and $\left(2 R, 3\right.$ ' $S, 8$ ' $\left.R, 9 a^{\prime} S\right)-8$ '-Methoxy-4-methylene-3'-[(2S)-4-methylene5-oxotetrahydrofuran-2-yl]hexahydro-3H-spiro\{furan-2,9'-pyrrolo[1,2-a]azepine\}-5,5'(4H, 6'H)-dione (21 and 22)

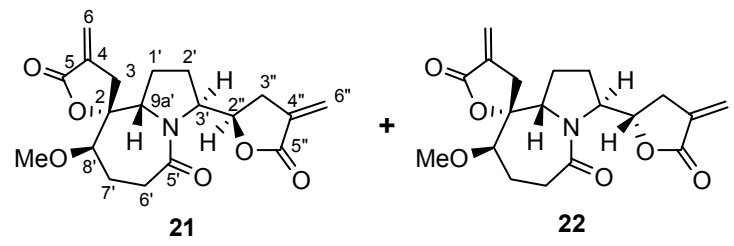

To a stirred solution of aldehyde 20 (460 mg, $1.57 \mathrm{mmol})$ in dry THF (16 mL) under $\mathrm{N}_{2}$ atmosphere, $\mathrm{Zn}(308 \mathrm{mg}, 4.71 \mathrm{mmol})$ was added. The solution was heated up and, when reflux started, a solution of ethyl 2-(bromomethyl)acrylate, 17, $(325 \mu \mathrm{L}, 2.35 \mathrm{mmol})$ in dry THF $(8 \mathrm{~mL})$ was added. After $1 \mathrm{~h}$ TLC analysis of the reaction mixture (EtOAc:MeOH 9:1) revealed the absence of starting aldehyde. The reaction mixture was filtered through Celite ${ }^{\circledR}$ and the organic solvent was evaporated under vacuum. The remaining colorless oil was purified by flash column chromatography on silica gel (EtOAc to EtOAc:MeOH 95:5) to provide the following fractions: i) bislactone erythro 21 (214 mg, $0.59 \mathrm{mmol}, 38 \%$ yield); a mixture of 21 and its threo isomer 22 (46 $\mathrm{mg}, 0.13 \mathrm{mmol}, 8 \%$ yield); iii) bislactone 21 (151 mg, $0.42 \mathrm{mmol}, 27 \%$ yield).

21 (erythro): ${ }^{1} \mathbf{H}$ NMR $\left(360 \mathrm{MHz}, \mathrm{CDCl}_{3}\right): \delta 6.25$ (t, $\left.J=3.0 \mathrm{~Hz}, 1 \mathrm{H}: \mathrm{H}_{6} / \mathrm{H}_{6}{ }^{\prime}\right), 6.24(\mathrm{t}, J=3.0 \mathrm{~Hz}$, $\left.1 \mathrm{H}: \mathrm{H}_{6} / \mathrm{H}_{6}{ }^{\prime}\right), 5.66$ (t, $\left.J=2.5,2 \mathrm{H}: \mathrm{H}_{6}, \mathrm{H}_{6}{ }^{\prime \prime}\right), 5.36$ (ddd, $\left.J=8.0,6.2,1.4 \mathrm{~Hz}, 1 \mathrm{H}: \mathrm{H}_{2},\right), 4.24$ (m, 2H: $\mathrm{H}_{3}$, 
$\mathrm{H}_{9 \mathrm{a}}$ ), $3.34\left(\mathrm{~s}, 3 \mathrm{H}: \mathrm{OCH}_{3}\right), 3.29\left(\mathrm{dd}, J=7.7,2.6 \mathrm{~Hz}, 1 \mathrm{H}: \mathrm{H}_{8}\right), 3.08$ (ddt, $J=17.8,8.5,2.7 \mathrm{~Hz}$, 1H: $\left.{ }_{3}{ }^{\prime}\right), 2.94$ (dt, $\left.J=18.2,2.9 \mathrm{~Hz}, 1 \mathrm{H}: \mathrm{H}_{3}\right), 2.81$ (ddd, $J=14.5,10.0,5.8 \mathrm{~Hz}, 1 \mathrm{H}: \mathrm{H}_{6}$ ), 2.70 (dt, $J=$ 18.2, $2.3 \mathrm{~Hz}, 1 \mathrm{H}: \mathrm{H}_{3}$ ), 2.51 (ddt, $\left.J=17.9,6.0,2.9 \mathrm{~Hz}, 1 \mathrm{H}: \mathrm{H}_{3}{ }^{\prime \prime}\right), 2.42$ (dt, $J=14.3,5.7 \mathrm{~Hz}, 1 \mathrm{H}: \mathrm{H}_{6}{ }^{\prime}$ ), 2.30 (tt, $\left.J=13.4,8.1 \mathrm{~Hz}, 1 \mathrm{H}: \mathrm{H}_{1}{ }^{\prime}\right), 2.04$ (dddd, $J=15.1,10.0,5.6,2.6 \mathrm{~Hz}, 1 \mathrm{H}: \mathrm{H}_{7}$ ), 1.91 (m, 2H: $\mathrm{H}_{2}$, $\mathrm{H}_{7^{\prime}}$ ), $1.74\left(\mathrm{dd}, J=13.3,7.7 \mathrm{~Hz}, 1 \mathrm{H}: \mathrm{H}_{1}{ }^{\prime}\right), 1.57$ (dd, $\left.J=13.3,8.1 \mathrm{~Hz}, 1 \mathrm{H}: \mathrm{H}_{2}{ }^{\prime}\right) ;{ }^{13} \mathbf{C}$ NMR $(125 \mathrm{MHz}$, $\left.\mathrm{CDCl}_{3}\right): \delta 172.5\left(\mathrm{C}_{5}{ }^{\prime}\right), 170.2\left(\mathrm{C}_{5} / \mathrm{C}_{5}{ }^{\prime}\right), 169.3\left(\mathrm{C}_{5} / \mathrm{C}_{5}{ }^{\prime}\right), 133.8\left(\mathrm{C}_{4} / \mathrm{C}_{4}{ }^{\prime}\right), 133.6\left(\mathrm{C}_{4} / \mathrm{C}_{4}{ }^{\prime}\right), 122.9$ $\left(\mathrm{C}_{6} / \mathrm{C}_{6^{\prime}}\right), 122.8\left(\mathrm{C}_{6} / \mathrm{C}_{6}{ }^{\prime}\right), 84.9\left(\mathrm{C}_{2}\right), 82.7\left(\mathrm{C}_{8^{\prime}}\right), 75.5\left(\mathrm{C}_{2}{ }^{\prime}\right), 62.0\left(\mathrm{C}_{3}\right.$, and $\left.\mathrm{C}_{9 \mathrm{a}^{\prime}}\right), 57.4\left(\mathrm{OCH}_{3}\right), 34.8$ $\left(\mathrm{C}_{3}\right), 30.7\left(\mathrm{C}_{6}\right), 30.2\left(\mathrm{C}_{3}{ }^{\prime}\right), 26.5\left(\mathrm{C}_{1}\right), 22.7\left(\mathrm{C}_{2}\right), 20.5\left(\mathrm{C}_{7}\right)$; ; HRMS $(\mathrm{CI}+)$ : calcd for $\mathrm{C}_{19} \mathrm{H}_{23} \mathrm{NNaO}_{6}$ : $384.1418[\mathrm{M}+\mathrm{Na}]^{+}$; found 384.1419 .

22 (threo): ${ }^{1} \mathbf{H}$ NMR (360 MHz, $\left.\mathrm{CDCl}_{3}\right): \delta 6.25$ (t, $\left.J=3.0 \mathrm{~Hz}, 1 \mathrm{H}: \mathrm{H}_{6}\right), 6.13$ (t, $\left.J=2.9 \mathrm{~Hz}, 1 \mathrm{H}: \mathrm{H}_{6}{ }^{\prime}\right)$, 5.67 (t, $\left.J=2.6 \mathrm{~Hz}, 1 \mathrm{H}: \mathrm{H}_{6}\right), 5.55$ (t, $\left.J=2.5 \mathrm{~Hz}, 1 \mathrm{H}: \mathrm{H}_{6}{ }^{\prime}\right), 4.98$ (ddd, $\left.J=8.5,5.1,3.5 \mathrm{~Hz}, 1 \mathrm{H}: \mathrm{H}_{2}{ }^{\prime}\right)$, $4.51\left(\mathrm{dt}, J=8.6,3.0 \mathrm{~Hz}, 1 \mathrm{H}: \mathrm{H}_{3}{ }^{\prime}\right), 4.18\left(\mathrm{dd}, J=8.9,1.9 \mathrm{~Hz}, 1 \mathrm{H}: \mathrm{H}_{9 \mathrm{a}}{ }^{\prime}\right), 3.32\left(\mathrm{~s}, 3 \mathrm{H}: \mathrm{OCH}_{3}\right), 3.25(\mathrm{dd}, J$ $\left.=7.1,2.6 \mathrm{~Hz}, 1 \mathrm{H}: \mathrm{H}_{8}{ }^{\prime}\right), 3.01$ (ddt, $\left.J=17.3,5.1,2.6 \mathrm{~Hz}, 1 \mathrm{H}: \mathrm{H}_{3}{ }^{\prime}\right), 2.91\left(\mathrm{dt}, J=18.3,3.0 \mathrm{~Hz}, 1 \mathrm{H}_{2} \mathrm{H}_{3}\right)$, 2.84 (ddt, $\left.J=17.3,8.4,2.8 \mathrm{~Hz}, 1 \mathrm{H}: \mathrm{H}_{3}{ }^{\prime}\right), 2.70$ (dt, $\left.J=18.3,2,6 \mathrm{~Hz}, 1 \mathrm{H}: \mathrm{H}_{3}\right), 2.68$ (m, 1H: $\left.\mathrm{H}_{6}{ }^{\prime}\right), 2.29$

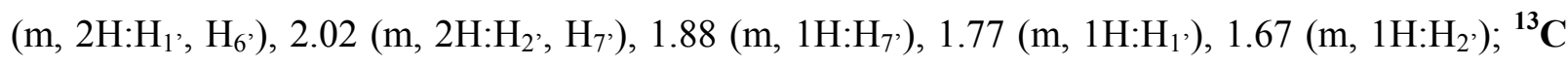
NMR (125 MHz, $\left.\mathrm{CDCl}_{3}\right)$ : $\delta 173.2\left(\mathrm{C}_{5}{ }^{\prime}\right), 170.2\left(\mathrm{C}_{5},\right), 169.1\left(\mathrm{C}_{5}\right), 134.2\left(\mathrm{C}_{4},\right), 133.7\left(\mathrm{C}_{4}\right), 123.0$ $\left(\mathrm{C}_{6}\right), 121.5\left(\mathrm{C}_{6}{ }^{\prime}\right), 84.6\left(\mathrm{C}_{2}\right), 82.6\left(\mathrm{C}_{8^{\prime}}\right), 78.4\left(\mathrm{C}_{2}{ }^{\prime}\right), 61.0\left(\mathrm{C}_{9 \mathrm{a}^{\prime}}\right), 60.4\left(\mathrm{C}_{3^{\prime}}\right), 57.3\left(\mathrm{OCH}_{3}\right), 35.4\left(\mathrm{C}_{3}\right)$, $\left.30.6\left(\mathrm{C}_{3^{\prime}}{ }^{\prime}\right), 30.2\left(\mathrm{C}_{6^{\prime}}\right), 26.5\left(\mathrm{C}_{2^{\prime}}\right), 26.4\left(\mathrm{C}_{1^{\prime}}\right), 20.3\left(\mathrm{C}_{7^{\prime}}\right)\right)$; HRMS (CI+): calcd for $\mathrm{C}_{19} \mathrm{H}_{23} \mathrm{NNaO}_{6}$ : $384.1418[\mathrm{M}+\mathrm{Na}]^{+}$; found 384.1408 .

$(2 R, 4 S, 3 ' S, 8$ ' $R, 9 \mathrm{a} ' S, 2$ '' $R, 4$ '' $R)-$

(23) and $\left(2 R, 4 R, 3 ' S, 8\right.$ ' $\left.R, 9 a^{\prime} S\right)-8$ '-Methoxy-4-methyl-3'[(2R,4R)-4-methyl-5-oxotetrahydrofuran-2-yl]hexahydro-3H-spiro\{furan-2,9'-pyrrolo[1,2a] azepine $\}-5\left(4 H, 6^{\prime} H\right)$-one (24)
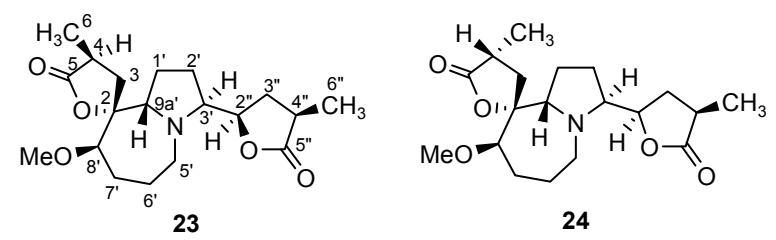

24

A solution of lactam $21(21 \mathrm{mg}, 58.1 \mu \mathrm{mol})$ in dry THF $(2 \mathrm{~mL})$ was added to a flask containing Lawesson's reagent $(26 \mathrm{mg}, 63.9 \mu \mathrm{mol})$ and the mixture was heated at reflux for $4.5 \mathrm{~h}$. At this time, TLC analysis (EtOAc) of the reaction mixture showed no starting material. The solvent was 
removed under vacuum and the resulting oil was filtered through $\mathrm{Al}_{2} \mathrm{O}_{3}$ Brockman I using EtOAc as eluent. Evaporation under reduced pressure of the organic solvent afforded a whitish solid (16 mg, $R_{\mathrm{f}}$ : 0.58). Raney-Ni (400 mg) was added to a solution of this solid in EtOH (2 mL) and the mixture was heated at reflux for $2 \mathrm{~h}$. Then, the mixture was filtered through Celite ${ }^{\circledR}$ and the solvent was evaporated in vacuo. The residue was purified by flash column chromatography on silica gel (EtOAc:hexanes 1:4 to 1:1) to give a mixture of the amines 23 and 24 (11 mg, $31.3 \mu \mathrm{mol}, 54 \%$ yield). By repeated chromatographies an enriched sample of $\mathbf{2 4}$ was isolated.

24: ${ }^{1} \mathbf{H}$ NMR (500 MHz, $\mathrm{CDCl}_{3}$ ): $\delta 4.71$ (ddd, $\left.J=10.7,5.9,2.1 \mathrm{~Hz}, 1 \mathrm{H}: \mathrm{H}_{2}{ }^{\prime}\right), 3.54$ (d, $J=9.9 \mathrm{~Hz}$, 1H: $\left.\mathrm{H}_{8^{\prime}}\right), 3.30$ (s, 3H:OCH${ }_{3}$ ), 3.27 (bd, $J=7.5 \mathrm{~Hz}, 1 \mathrm{H}: \mathrm{H}_{3}{ }^{\prime}$ ), 3.19 (ddd, $J=12.2,5.6,2.2 \mathrm{~Hz}, 1 \mathrm{H}^{\circ} \mathrm{H}_{5}{ }^{\prime}$ ),

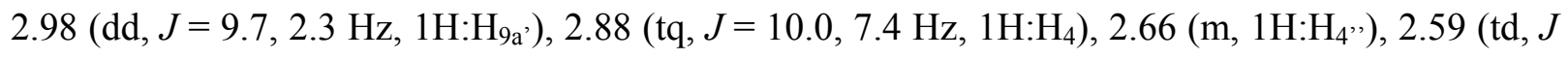
$\left.=12.2,3.8 \mathrm{~Hz}, 1 \mathrm{H}: \mathrm{H}_{5}{ }^{\prime}\right), 2.48$ (dd, $\left.J=13.0,10.1,1 \mathrm{H}: \mathrm{H}_{3}\right), 2.35$ (ddd, $\left.J=12.7,9.0,5.9 \mathrm{~Hz}, 1 \mathrm{H}: \mathrm{H}_{3}{ }^{\prime}\right)$,

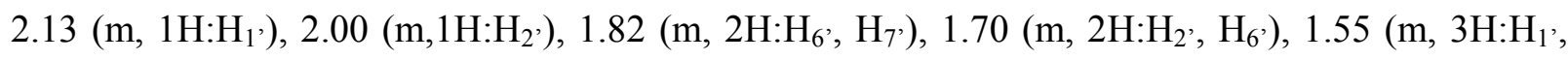
$\left.\mathrm{H}_{7^{\prime}}, \mathrm{H}_{3^{\prime}}\right), 1.28\left(\mathrm{~d}, J=7.1 \mathrm{~Hz}, 3 \mathrm{H}: 3 \mathrm{H}_{6}{ }^{\prime}\right), 1.26\left(\mathrm{~m}, 1 \mathrm{H}: \mathrm{H}_{3}\right), 1.20\left(\mathrm{~d}, J=7.4 \mathrm{~Hz}, 3 \mathrm{H}: 3 \mathrm{H}_{6}\right) ;{ }^{13} \mathbf{C} \mathbf{N M R}$ $\left(125 \mathrm{MHz}, \mathrm{CDCl}_{3}\right) \delta 181.0\left(\mathrm{C}_{5}\right), 179.2\left(\mathrm{C}_{5}{ }^{\prime}\right), 91.0\left(\mathrm{C}_{2}\right), 87.6\left(\mathrm{C}_{8^{\prime}}\right), 76.9\left(\mathrm{C}_{2},\right), 70.0\left(\mathrm{C}_{9 \mathrm{a}^{\prime}}\right), 66.5$ $\left(\mathrm{C}_{3}{ }^{\prime}\right), 57.5\left(\mathrm{OCH}_{3}\right), 51.1\left(\mathrm{C}_{5}{ }^{\prime}\right), 35.8\left(\mathrm{C}_{4}\right), 35.5\left(\mathrm{C}_{4}{ }^{\prime}\right), 35.3\left(\mathrm{C}_{3}\right), 34.2\left(\mathrm{C}_{3^{\prime}}{ }^{\prime}\right), 28.7\left(\mathrm{C}_{6}{ }^{\prime}\right), 26.8\left(\mathrm{C}_{7^{\prime}}\right)$, $25.6\left(\mathrm{C}_{1}\right), 25.3\left(\mathrm{C}_{2}{ }^{\prime}\right), 16.8\left(\mathrm{C}_{6}\right), 15.2\left(\mathrm{C}_{6}{ }^{\prime}\right)$.

23: ${ }^{1} \mathbf{H}-\mathbf{N M R}\left(500 \mathrm{MHz}, \mathrm{CDCl}_{3}\right.$, mixture of 23 and 24): see Figure below; ${ }^{13} \mathbf{C}$ NMR (125 MHz, $\mathrm{CDCl}_{3}$, data extracted from a mixture of $\mathbf{2 3}$ and 24) $\delta 180.8,179.3,91.4,86.2,69.9,66.2,57.3$, $50.9,35.4,35.0,34.1,33.5,27.8,26.7,25.6,25.4,16.4,15.2$.

$\left(2 R, 4 S, 3 ' S, 8\right.$ ' $R, 9 a^{\prime} S, 2$ '' $S, 4$ '' $\left.R\right)-\quad(25) \quad$ and $\quad(2 R, 4 R, 3 ' S, 8$ ' $R, 9$ a' $S)-8$ '-Methoxy-4-methyl-3'[(2S,4R)-4-methyl-5-oxotetrahydrofuran-2-yl]hexahydro-3H-spiro\{furan-2,9'-pyrrolo[1,2-a]azepine\}-5,5'(4H,6' $H)$-dione (26)
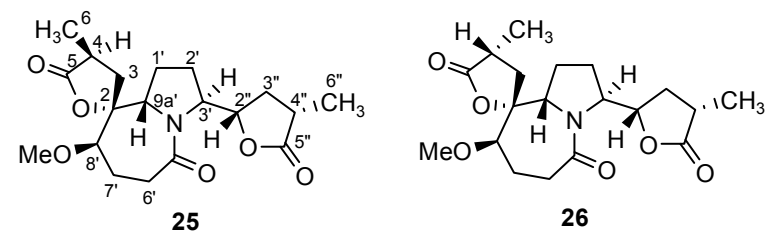

To a solution of bislactone $22(39 \mathrm{mg}, 0.108 \mathrm{mmol})$ in a 9:1 mixture of $\mathrm{EtOH}$ and $2 \mathrm{M} \mathrm{HCl}(3 \mathrm{~mL})$, $\mathrm{Pd} / \mathrm{C}(40 \mathrm{mg})$ was added. The reaction was stirred in a 6 bar atmosphere of $\mathrm{H}_{2}$ for $23 \mathrm{~h}$. Then, it was filtered through Celite ${ }^{\circledR}$ and the filtrate was washed with saturated solution of $\mathrm{NaHCO}_{3}$. The 
aqueous layer was extracted with $\mathrm{CH}_{2} \mathrm{Cl}_{2}(3 \times 5 \mathrm{~mL})$. The combined organic extracts were dried over anhydrous $\mathrm{Na}_{2} \mathrm{SO}_{4}$ and concentrated under reduced pressure. The residue was purified by flash column chromatography (EtOAc:hexanes 1:1 to 5:1) to give a mixture of lactones $\mathbf{2 5}$ and 26 (27 mg, $73.9 \mu \mathrm{mol}, 68 \%$ yield): ${ }^{1} \mathbf{H}$ NMR (500 MHz, $\mathrm{CDCl}_{3}$ ): see Figure below.

\section{$\left(2 R, 4 R, 3 ' S, 8\right.$ ' $\left.R, 9 a^{\prime} S\right)-8$ '-Methoxy-4-methyl-3'-[(2S,4S)-4-methyl-5-oxotetrahydrofuran-2-yl] hexahydro-3H-spiro\{furan-2,9'-pyrrolo[1,2-a] azepine\}-5(4H,6' $H)$-one (2) and its $(4 S)$ epimer (epi-2)}

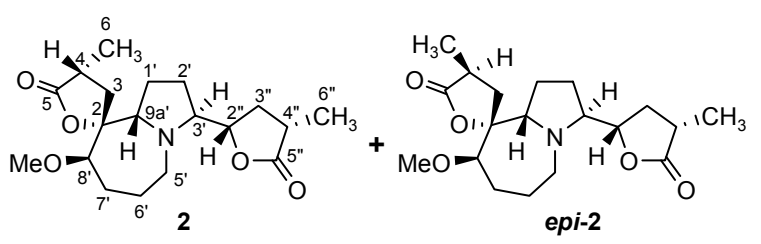

Lawesson's reagent (30 mg, $73.9 \mu \mathrm{mol}$ ) was added to a solution of a mixture of $\mathbf{2 5}$ and $\mathbf{2 6}(27 \mathrm{mg}$, $73.9 \mu \mathrm{mol})$ in dry THF $(3 \mathrm{~mL})$ and the mixture was heated at the reflux temperature for $4.5 \mathrm{~h}$. At this time, TLC analysis (EtOAc) of the reaction mixture showed no starting lactam. The solvent was removed under vacuum and the residue was filtered through $\mathrm{Al}_{2} \mathrm{O}_{3}$ Brockman I using EtOAc as eluent. Evaporation of the solvent under vacuum afforded a white solid (20 mg), which was dissolved in EtOH (3 mL). Then Raney-Ni $(400 \mathrm{mg})$ was added and the reaction mixture was heated at reflux for $2 \mathrm{~h}$. Then, it was filtered through Celite ${ }^{\circledR}$ and the solvent was evaporated in vacuo. Purification of the residue by flash column chromatography on silica gel (EtOAc:hexanes 2:1) furnished a mixture of 2 and $\boldsymbol{e p i - 2}(11 \mathrm{mg}, 31.3 \mu \mathrm{mol}, 45 \%$ yield for the two steps). Repeated column chromatography provided analytical samples of each diastereomer.

2: first eluted; $[\alpha]_{\mathrm{D}}{ }^{20}=-16\left(c 0.25\right.$, acetone), $[\alpha]_{\mathrm{D}}{ }^{20}=-30\left(c 0.25, \mathrm{CH}_{2} \mathrm{Cl}_{2}\right)$; IR (ATR): 2960, 2958, 2924, 2886, 2855, 2825, 1750, 1453, 1441, 1256, 1230, 1222, 1172, 1111, 1088, 1008; ${ }^{1} \mathbf{H}$ NMR (500 MHz, $\mathrm{CDCl}_{3}$ ): $\delta 4.31$ (ddd, $\left.J=10.9,8.2,5.4 \mathrm{~Hz}, 1 \mathrm{H}: \mathrm{H}_{2}{ }^{\prime}\right), 3.57$ (m, 1H: $\mathrm{H}_{5}$ ), 3.55 (d, $J=9.8$

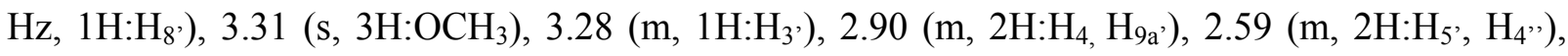
$2.52\left(\mathrm{dd}, J=12.9,10.1 \mathrm{~Hz}, 1 \mathrm{H}: \mathrm{H}_{3}\right), 2.38$ (ddd, $\left.J=12.8,8.3,5.4 \mathrm{~Hz}, 1 \mathrm{H}: \mathrm{H}_{3}{ }^{\prime}\right), 2.05$ (tt, $J=12.4,7.2$

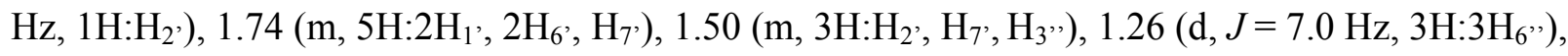
$1.26\left(\mathrm{~m}, 1 \mathrm{H}: \mathrm{H}_{3}\right), 1.22\left(\mathrm{~d}, J=7.4 \mathrm{~Hz}, 3 \mathrm{H}: 3 \mathrm{H}_{6}\right) ;{ }^{13} \mathbf{C} \mathbf{~ N M R}\left(125 \mathrm{MHz}, \mathrm{CDCl}_{3}\right) \delta 180.8\left(\mathrm{C}_{5}\right), 179.5$ $\left(\mathrm{C}_{5}{ }^{\prime}\right), 90.8\left(\mathrm{C}_{2}\right), 87.2\left(\mathrm{C}_{8^{\prime}}\right), 79.1\left(\mathrm{C}_{2}{ }^{\prime \prime}\right), 69.3\left(\mathrm{C}_{4}\right), 68.5\left(\mathrm{C}_{3}{ }^{\prime}\right), 57.5\left(\mathrm{OCH}_{3}\right), 52.1\left(\mathrm{C}_{5}{ }^{\prime}\right), 35.69$ 
$\left(\mathrm{C}_{3} / \mathrm{C}_{9 \mathrm{a}^{\prime}} / \mathrm{C}_{3^{\prime}}{ }^{\prime}\right), 35.67\left(\mathrm{C}_{3} / \mathrm{C}_{9 \mathrm{a}^{\prime}} / \mathrm{C}_{3^{\prime}}{ }^{\prime}\right), 35.60\left(\mathrm{C}_{3} / \mathrm{C}_{9 \mathrm{a}^{\prime}} / \mathrm{C}_{3^{\prime}}{ }^{\prime}\right), 34.8\left(\mathrm{C}_{4}{ }^{\prime}\right), 28.3\left(\mathrm{C}_{6}{ }^{\prime}\right), 26.9\left(\mathrm{C}_{2}{ }^{\prime}, \mathrm{C}_{7}{ }^{\prime}\right), 25.3$ $\left.\left(\mathrm{C}_{1}\right), 16.8\left(\mathrm{C}_{6}\right), 14.8\left(\mathrm{C}_{6}{ }^{\prime}\right)\right)$; HRMS $(\mathrm{CI}+)$ : calcd for $\mathrm{C}_{19} \mathrm{H}_{30} \mathrm{NO}_{5}: 352.2118[\mathrm{M}+\mathrm{H}]^{+}$; found 352.2124 .

Epi-2: second eluted; $[\alpha]_{\mathrm{D}}{ }^{20}=-60$ (c 0.09, acetone); IR (ATR): 2959, 2928, 2851, 2823, 1757, 1450, 1375, 1334, 1320, 1260, 1236, 1155, 1115, 1008; ${ }^{1} \mathbf{H}$ NMR (500 MHz, $\left.\mathrm{CDCl}_{3}\right): \delta 4.30$ (ddd, $\left.J=10.8,8.2,5.4 \mathrm{~Hz}, 1 \mathrm{H}: \mathrm{H}_{2}{ }^{\prime}\right), 3.52\left(\mathrm{~m}, 1 \mathrm{H}^{\prime} \mathrm{H}_{5}{ }^{\prime}\right), 3.48$ (dd, $\left.J=9.7,1.1 \mathrm{~Hz}, 1 \mathrm{H}: \mathrm{H}_{8}{ }^{\prime}\right), 3.34$ (s, $\left.3 \mathrm{H}: \mathrm{OCH}_{3}\right), 3.27\left(\mathrm{dt}, J=7.6,2.4 \mathrm{~Hz}, 1 \mathrm{H}: \mathrm{H}_{3}{ }^{3}\right), 3.04\left(\mathrm{dd}, J=9.0,2.7 \mathrm{~Hz}, 1 \mathrm{H}: \mathrm{H}_{9 \mathrm{a}}\right), 2.70\left(\mathrm{~m}, 2 \mathrm{H}: \mathrm{H}_{4}\right.$, $\left.\mathrm{H}_{5}{ }^{\prime}\right), 2.58$ (m, 1H: $\mathrm{H}_{4}$ ) $), 2.37$ (ddd, $\left.J=12.7,8.4,5.4 \mathrm{~Hz}, 1 \mathrm{H}: \mathrm{H}_{3}{ }^{\prime}\right), 2.05$ (dd, $J=13.2,6.3 \mathrm{~Hz}$, 1H: $\left.\mathrm{H}_{3}\right), 2.01$ (m, 1H: $\mathrm{H}_{2}$ ), 1.92 (dd, $\left.J=13.3,12.0 \mathrm{~Hz}, 1 \mathrm{H}: \mathrm{H}_{3}\right), 1.84$ (m, 2H: $\mathrm{H}_{1}, \mathrm{H}_{7}$ ) 1.73 (m,

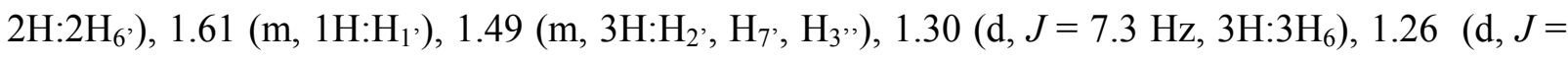
$\left.7.0 \mathrm{~Hz}, 3 \mathrm{H}: 3 \mathrm{H}_{6}{ }^{\prime}\right) ;{ }^{13} \mathbf{C}$ NMR (125 MHz, $\left.\mathrm{CDCl}_{3}\right) \delta 180.6\left(\mathrm{C}_{5} / \mathrm{C}_{5}{ }^{\prime \prime}\right), 179.5\left(\mathrm{C}_{5} / \mathrm{C}_{5}{ }^{\prime \prime}\right), 91.3\left(\mathrm{C}_{2}\right), 85.9$ $\left(\mathrm{C}_{8^{\prime}}\right), 79.6\left(\mathrm{C}_{2}{ }^{\prime}\right), 69.3\left(\mathrm{C}_{9 \mathrm{a}^{\prime}}\right), 68.1\left(\mathrm{C}_{3^{\prime}}\right), 57.4\left(\mathrm{OCH}_{3}\right), 51.8\left(\mathrm{C}_{5^{\prime}}\right), 35.5\left(\mathrm{C}_{3^{\prime}}{ }^{\prime}\right), 35.0\left(\mathrm{C}_{4} / \mathrm{C}_{4}{ }^{\prime}\right), 34.9$ $\left(\mathrm{C}_{4} / \mathrm{C}_{4}{ }^{\prime}\right), 33.9\left(\mathrm{C}_{3}\right), 27.4\left(\mathrm{C}_{6}\right), 26.83\left(\mathrm{C}_{2}, \mathrm{C}_{7}\right), 26.79\left(\mathrm{C}_{2}, \mathrm{C}_{7^{\prime}}\right), 25.4\left(\mathrm{C}_{1}\right), 16.4\left(\mathrm{C}_{6}\right), 14.8\left(\mathrm{C}_{6}{ }^{\prime}\right)$; HRMS (CI+): calcd for $\mathrm{C}_{19} \mathrm{H}_{30} \mathrm{NO}_{5}: 352.2118[\mathrm{M}+\mathrm{H}]^{+}$; found 352.2117 . 


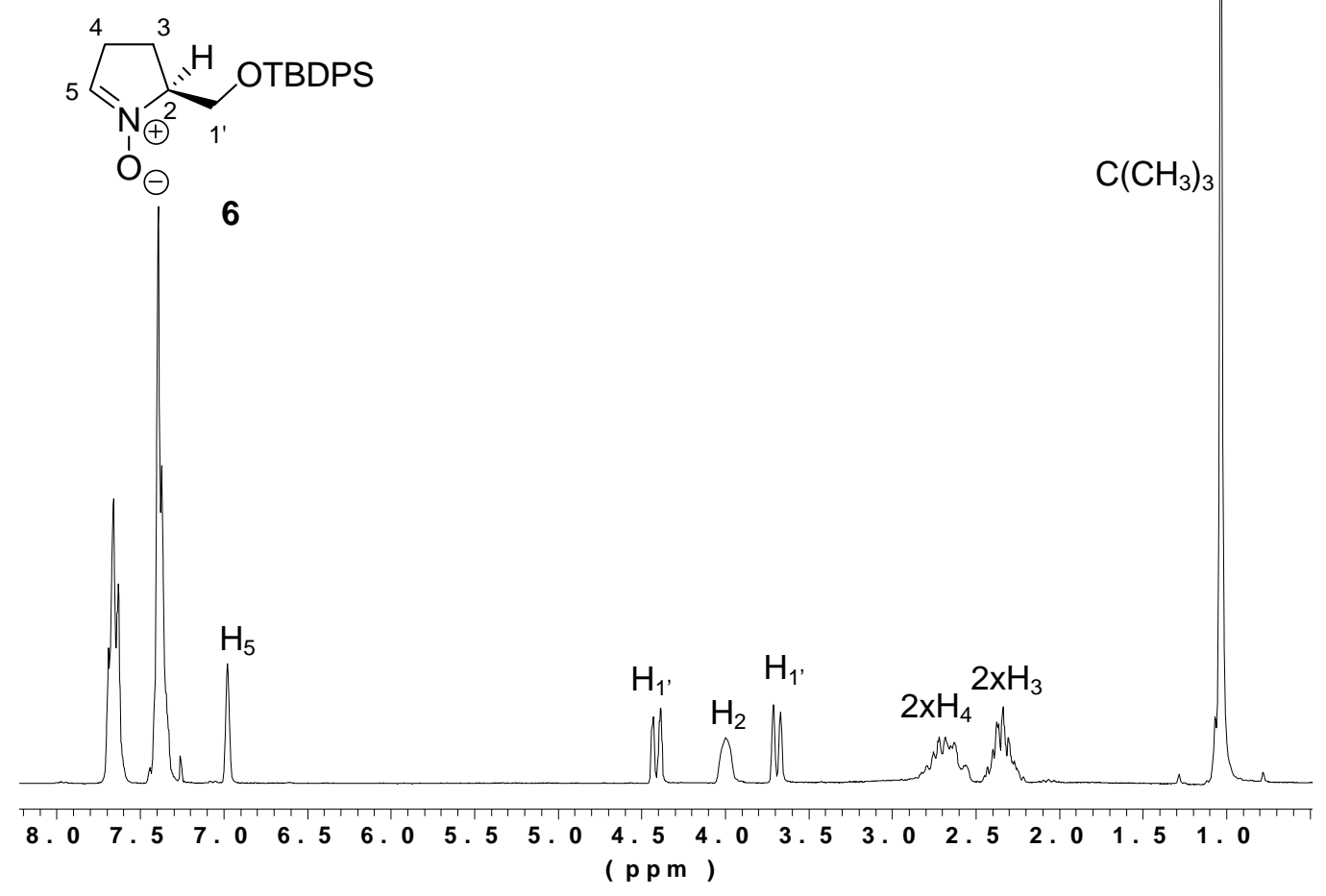

${ }^{1} \mathrm{H}-\mathrm{NMR}\left(\mathrm{CDCl}_{3}, 250 \mathrm{MHz}\right)$

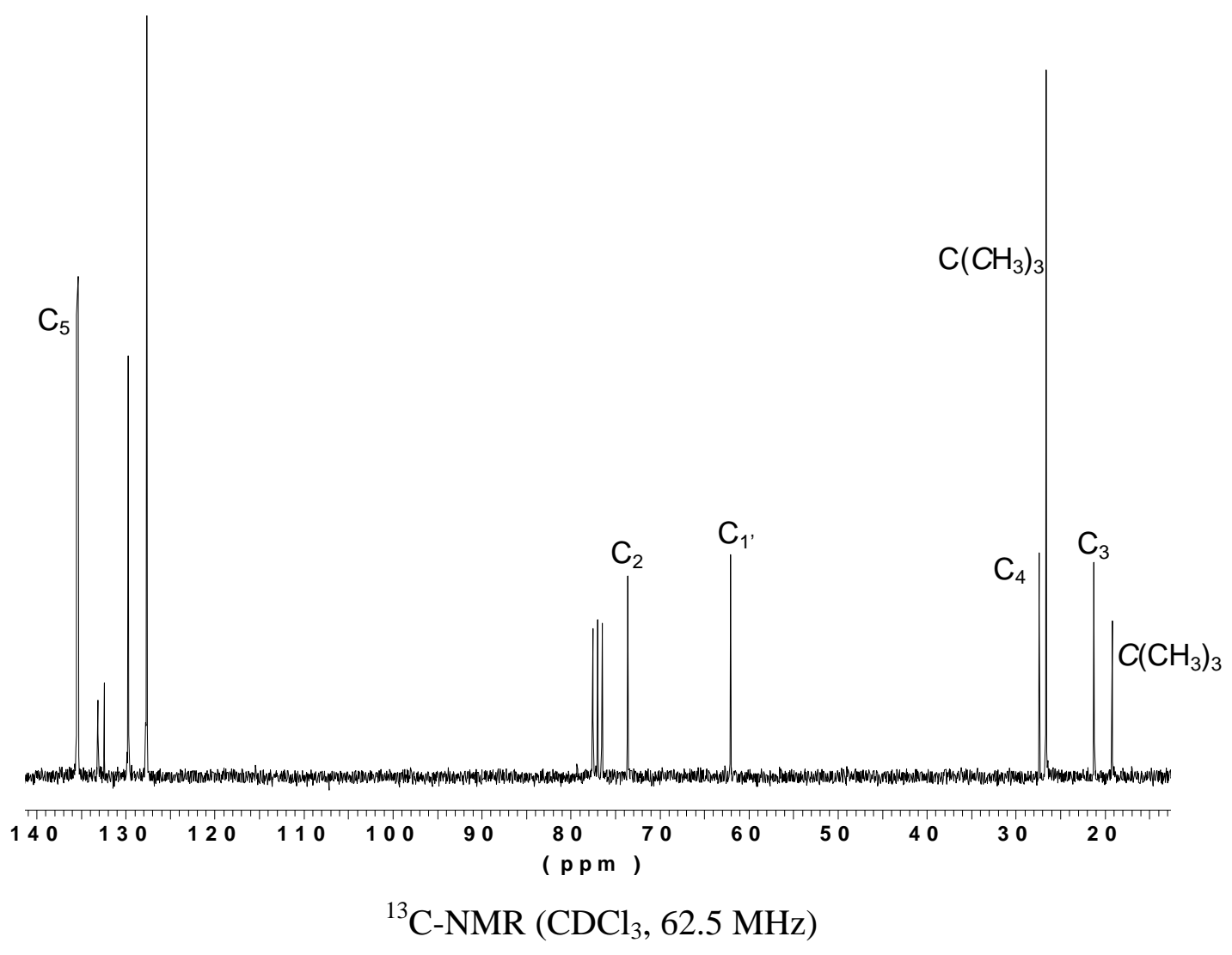




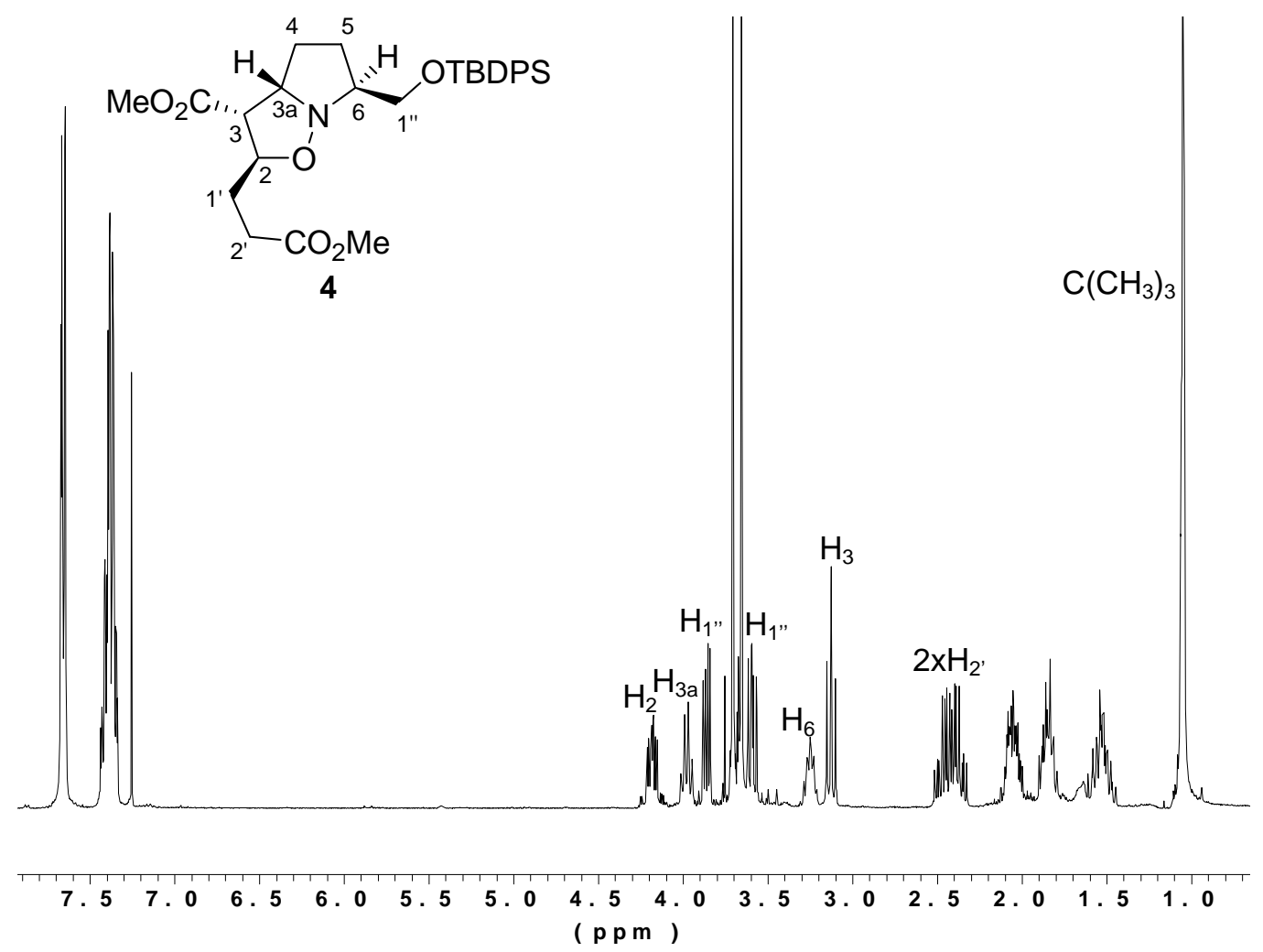

${ }^{1} \mathrm{H}-\mathrm{NMR}\left(\mathrm{CDCl}_{3}, 360 \mathrm{MHz}\right)$

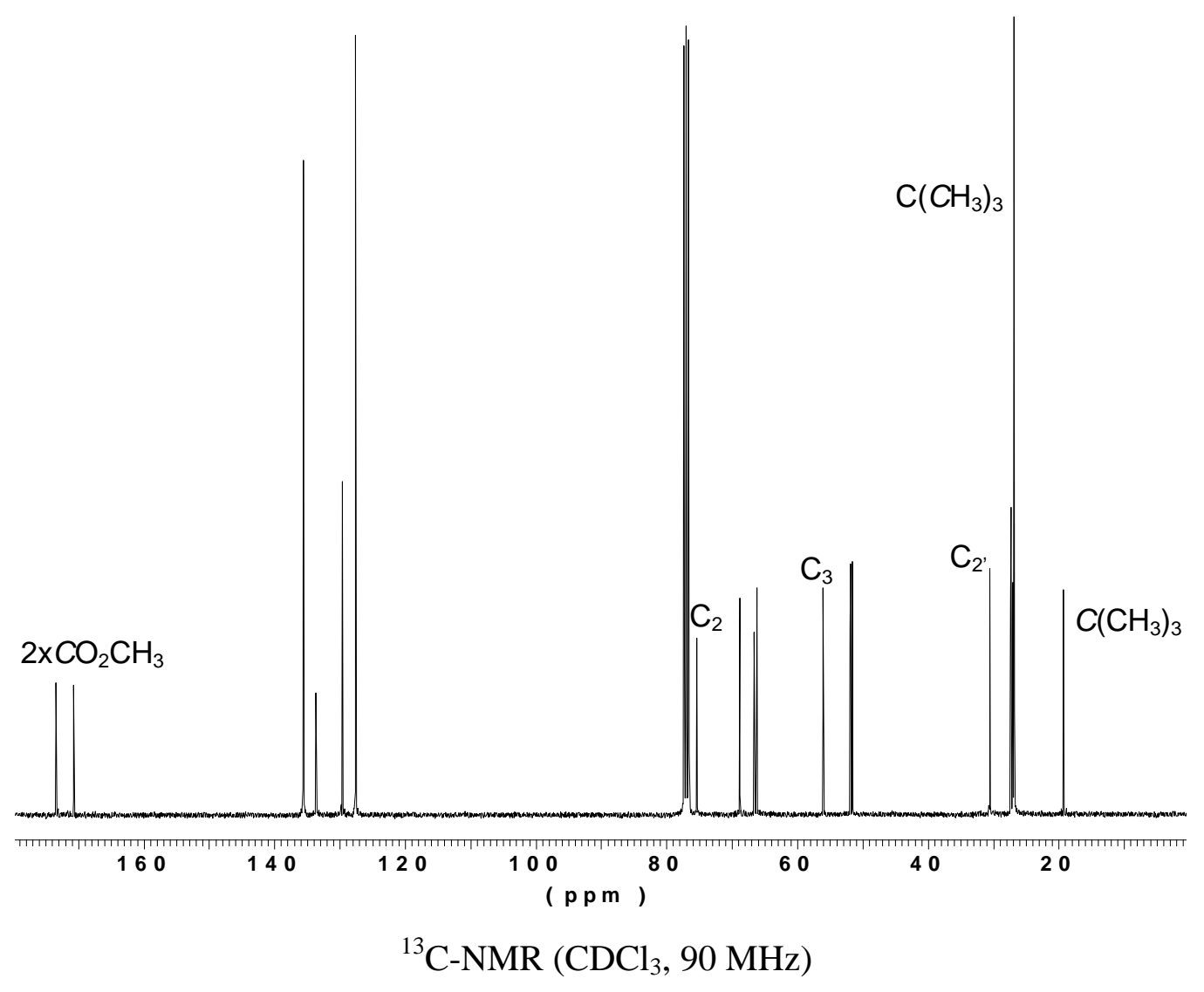



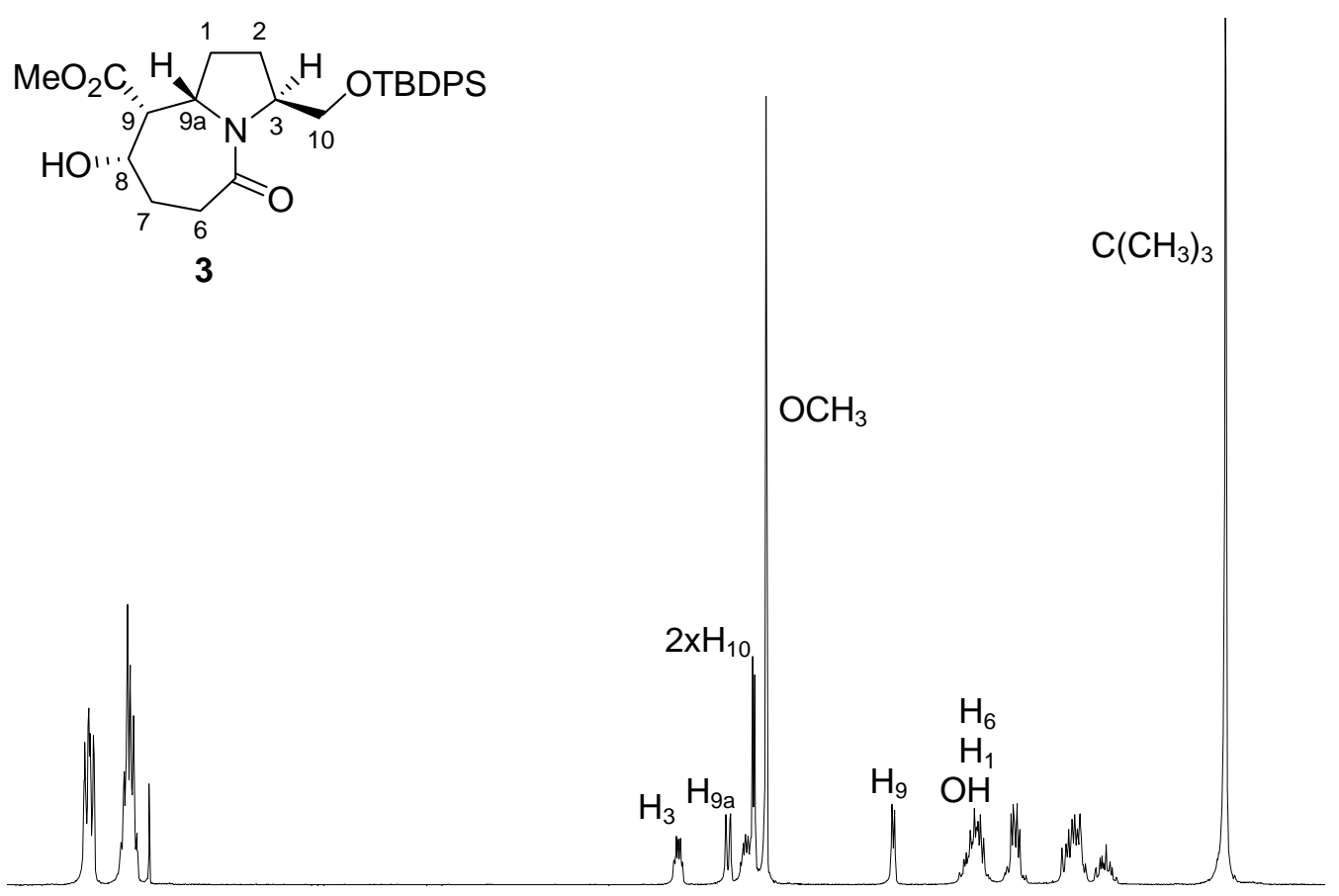

$\begin{array}{cccccccccccccccc}8.0 & 7.5 & 7.0 & 6.5 & 6.0 & 5.5 & 5.0 & 4.5 & 4.0 & 3.5 & 3.0 & 2.5 & 2.0 & 1.5 & 1.0 \\ (\mathrm{ppm}) & \end{array}$

${ }^{1} \mathrm{H}-\mathrm{NMR}\left(\mathrm{CDCl}_{3}, 360 \mathrm{MHz}\right)$

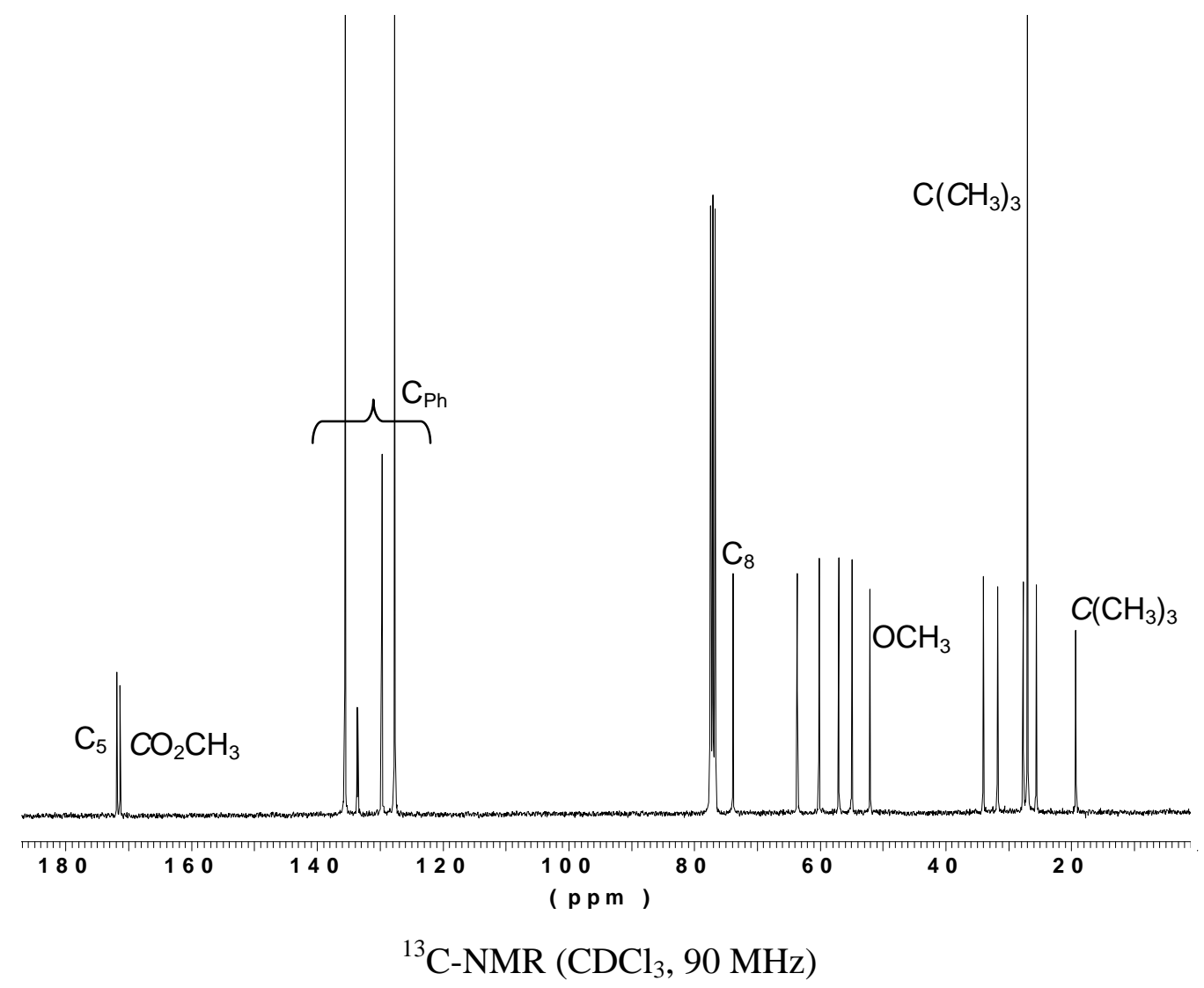



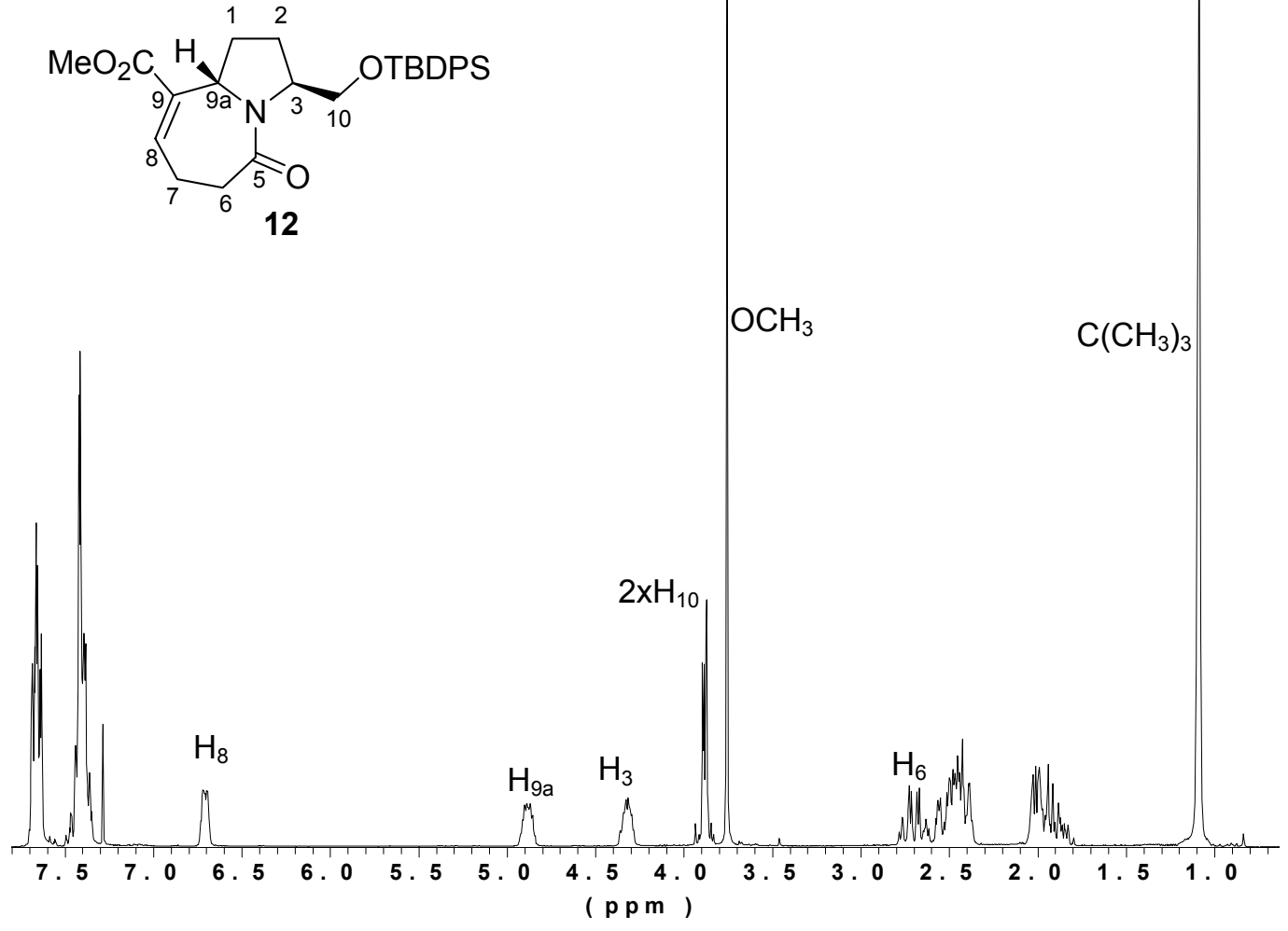

${ }^{1} \mathrm{H}-\mathrm{NMR}\left(\mathrm{CDCl}_{3}, 250 \mathrm{MHz}\right)$

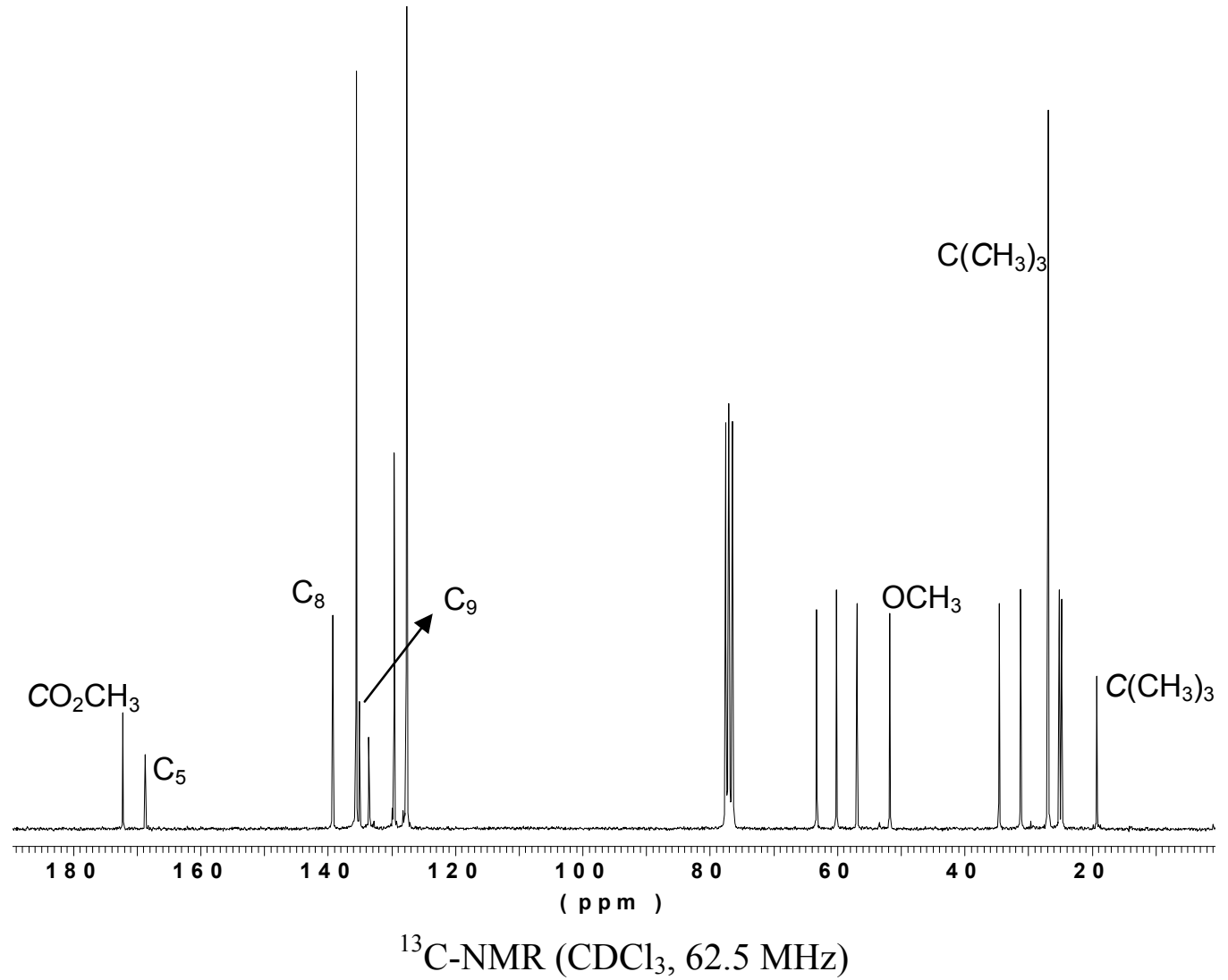



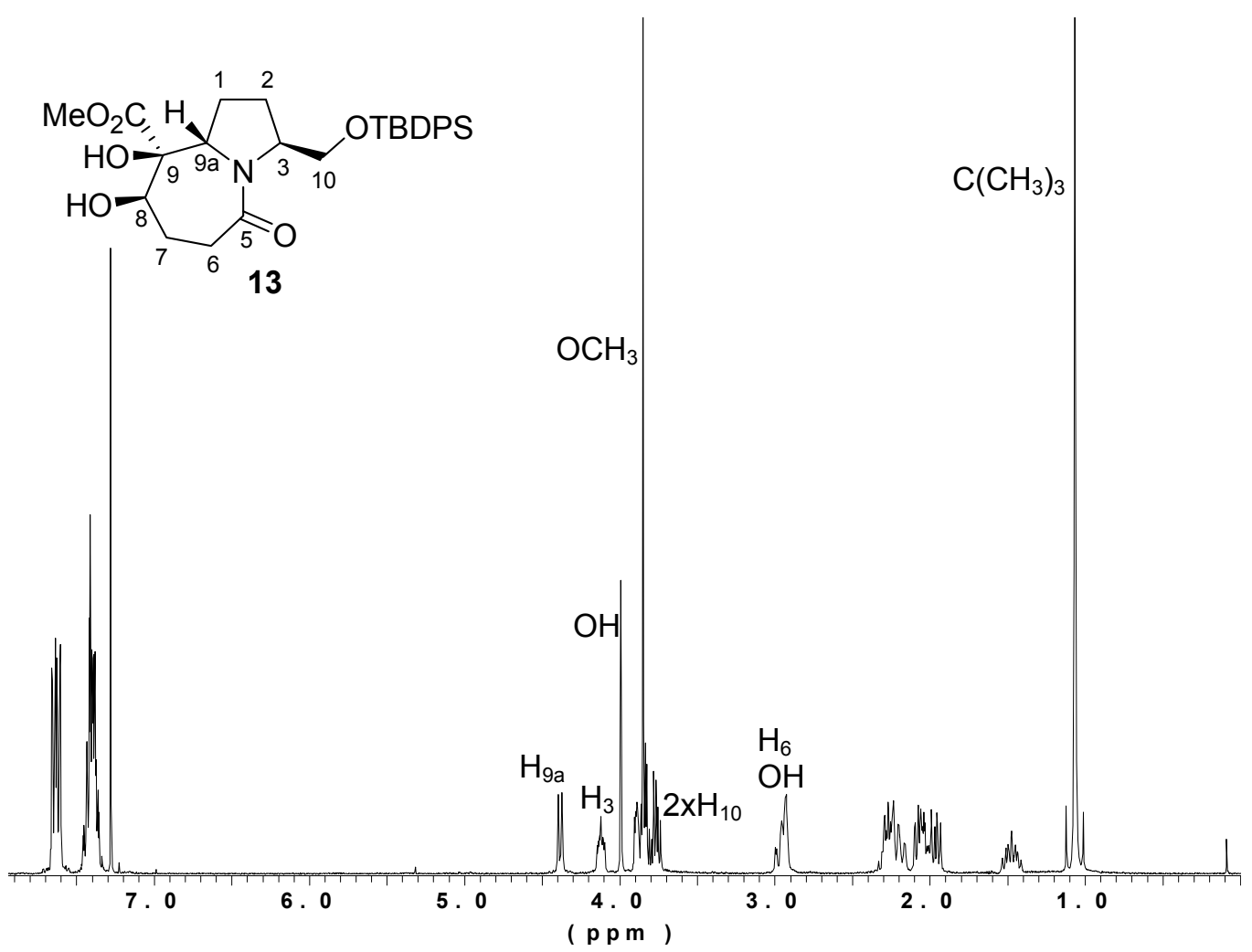

${ }^{1} \mathrm{H}-\mathrm{NMR}\left(\mathrm{CDCl}_{3}, 360 \mathrm{MHz}\right)$

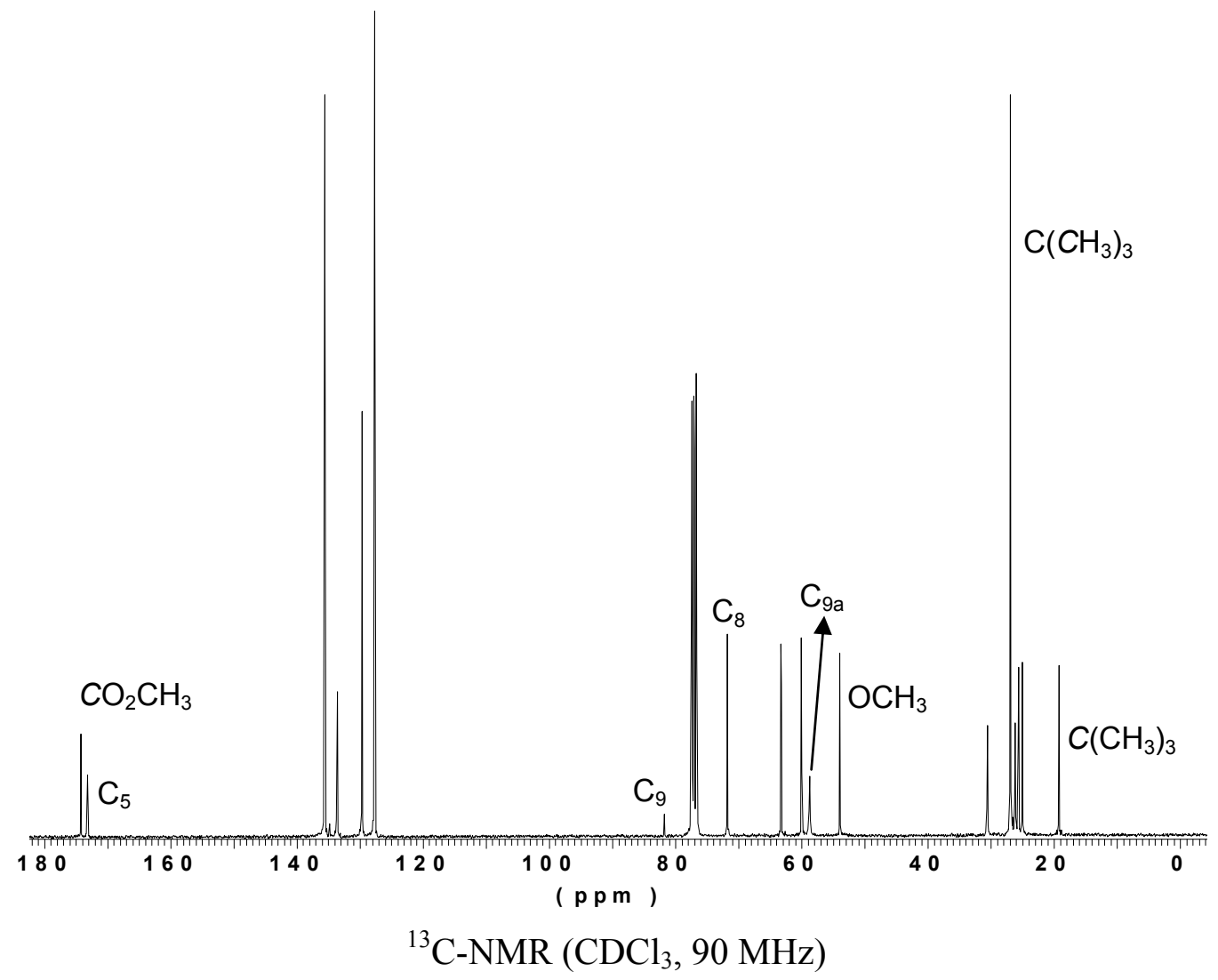




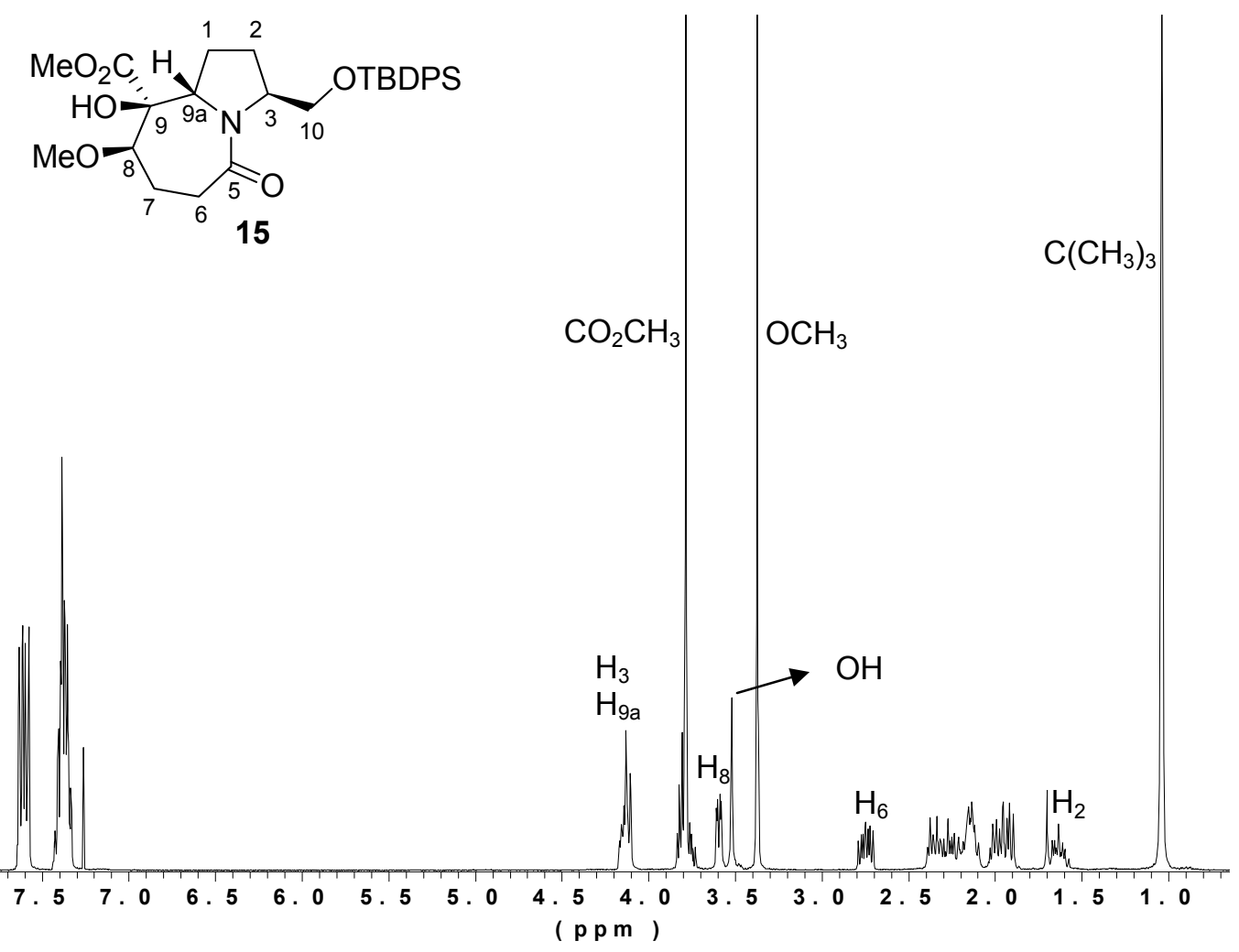

${ }^{1} \mathrm{H}-\mathrm{NMR}\left(\mathrm{CDCl}_{3}, 360 \mathrm{MHz}\right)$

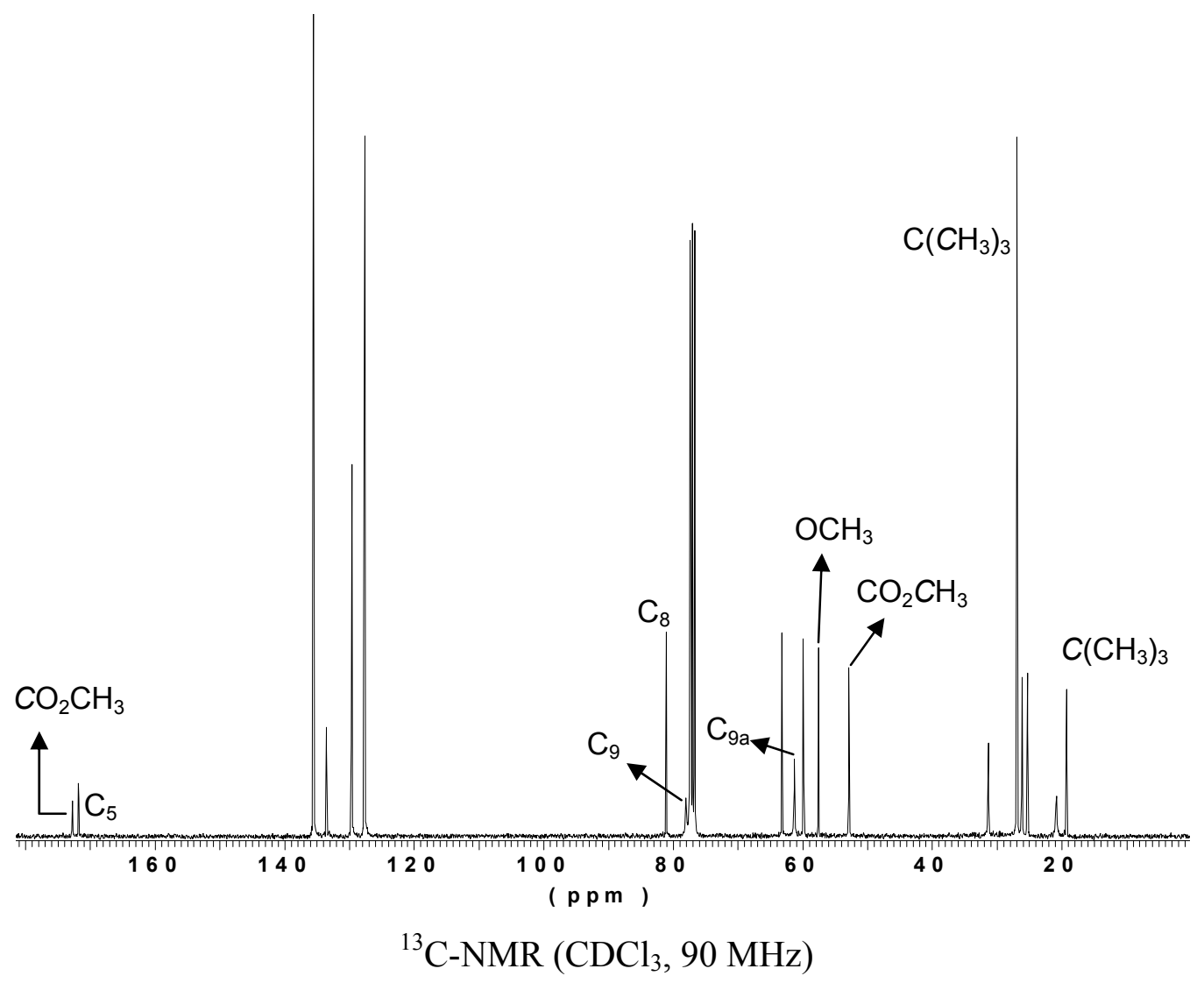



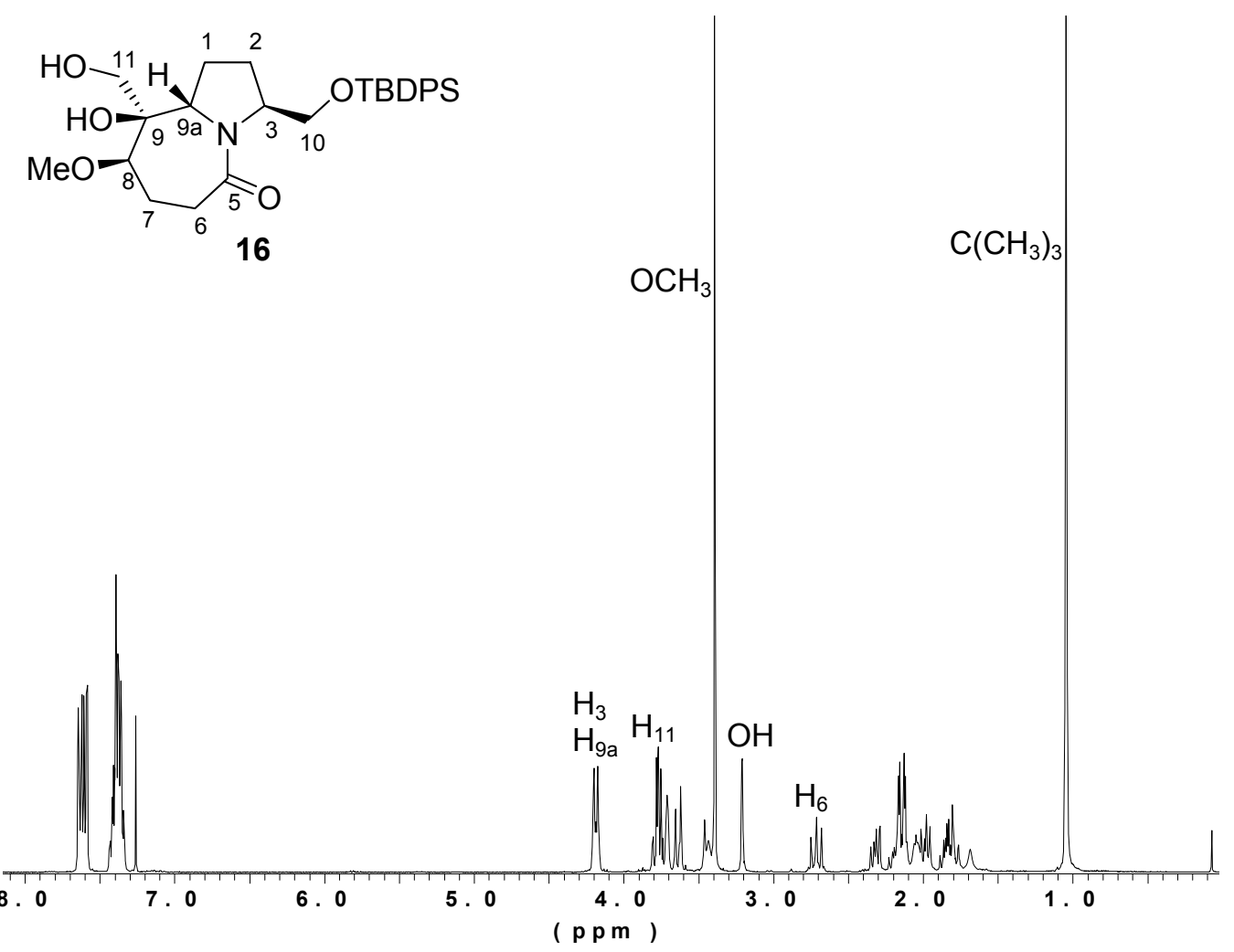

${ }^{1} \mathrm{H}-\mathrm{NMR}\left(\mathrm{CDCl}_{3}, 360 \mathrm{MHz}\right)$

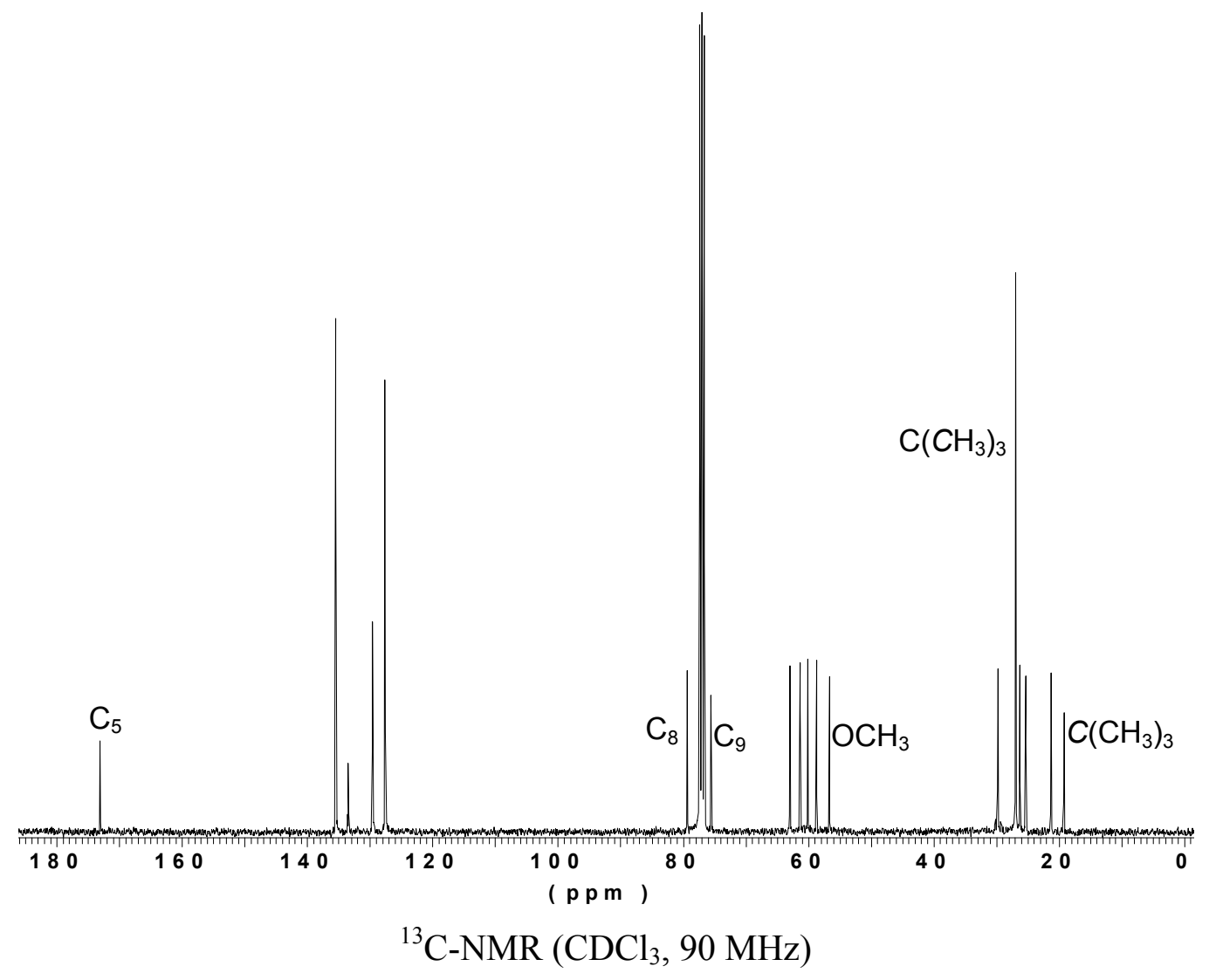



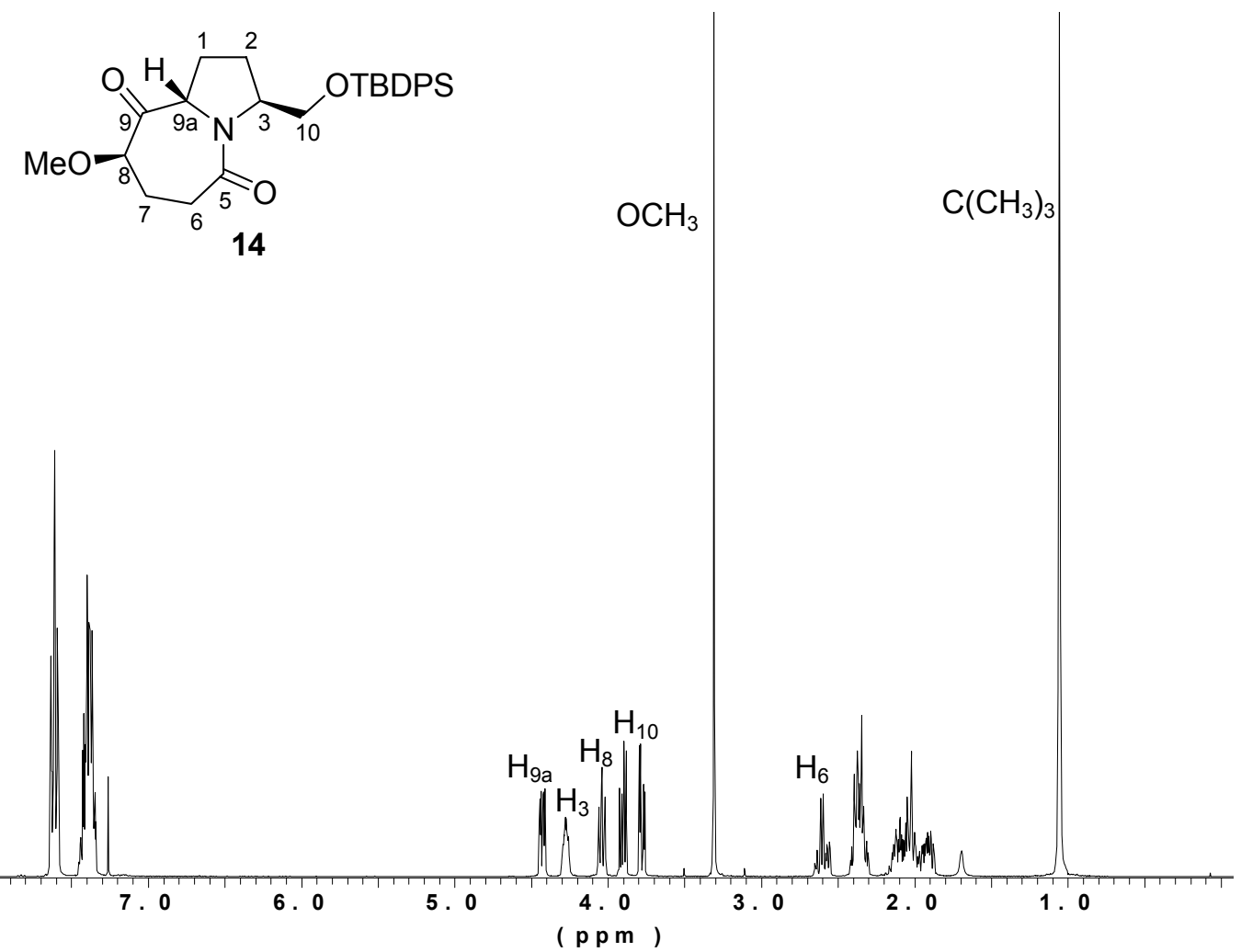

${ }^{1} \mathrm{H}-\mathrm{NMR}\left(\mathrm{CDCl}_{3}, 360 \mathrm{MHz}\right)$

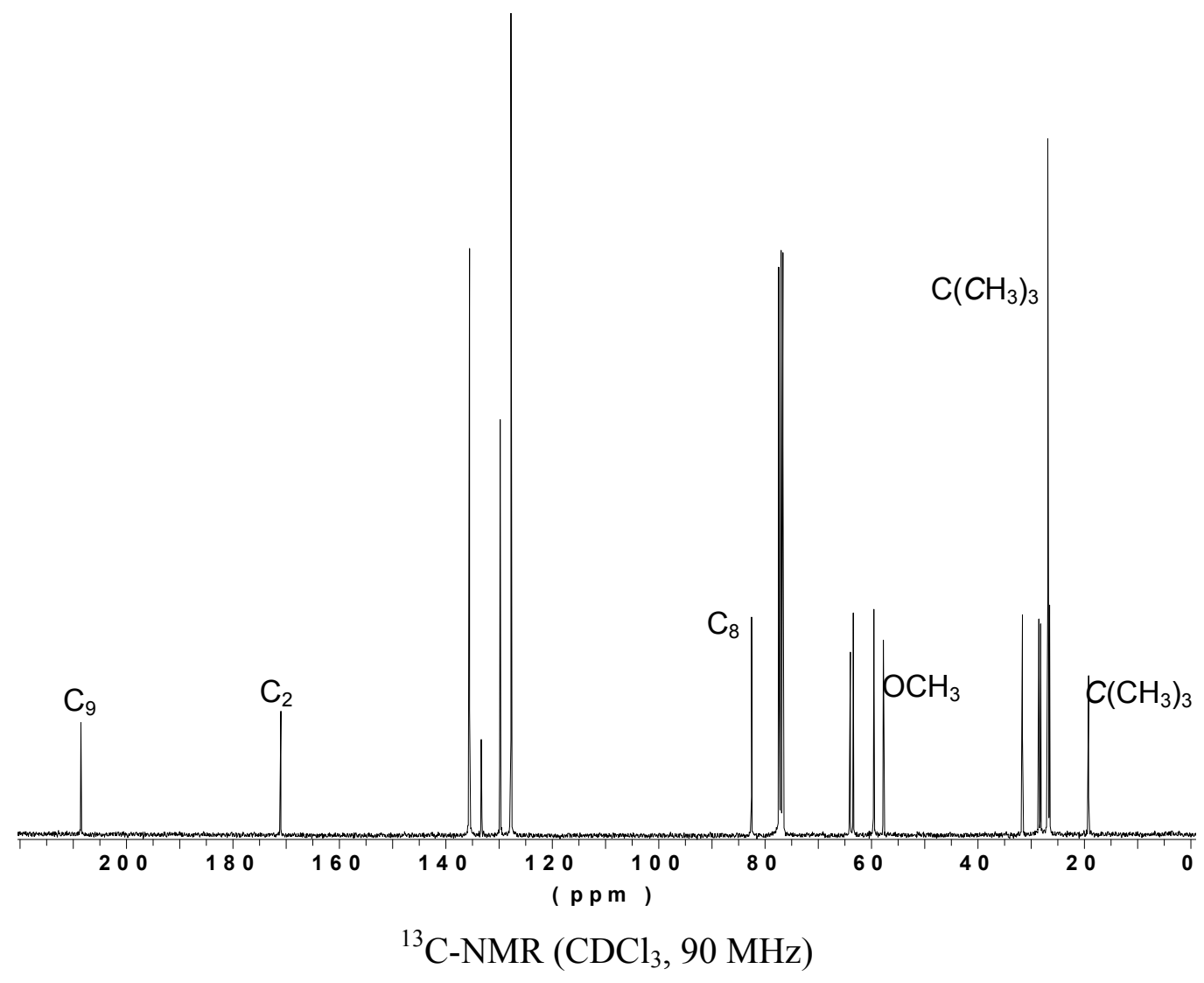




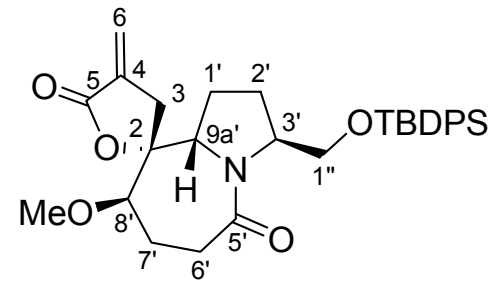

18

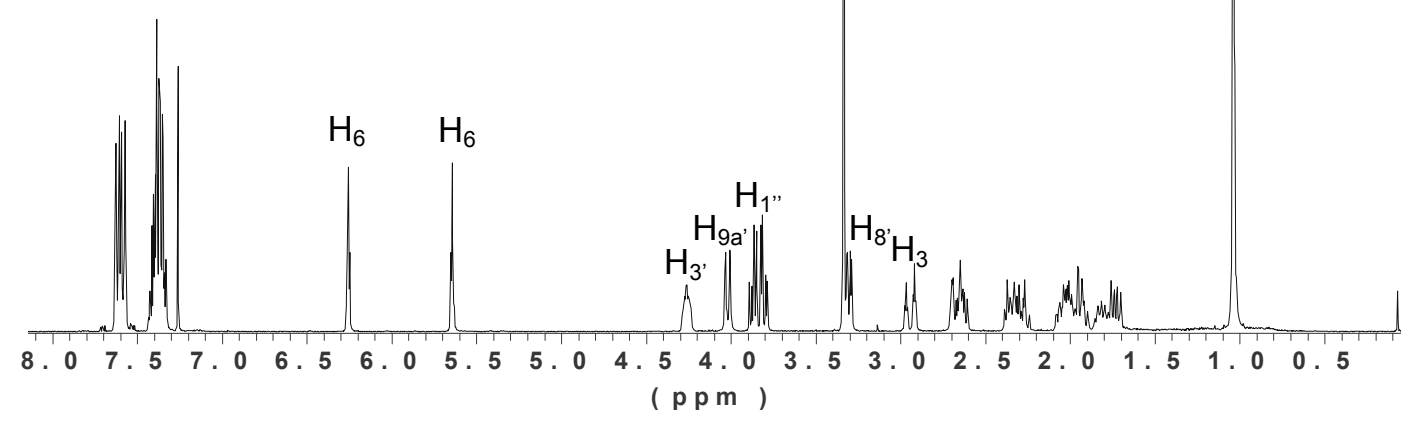

${ }^{1} \mathrm{H}-\mathrm{NMR}\left(\mathrm{CDCl}_{3}, 360 \mathrm{MHz}\right)$

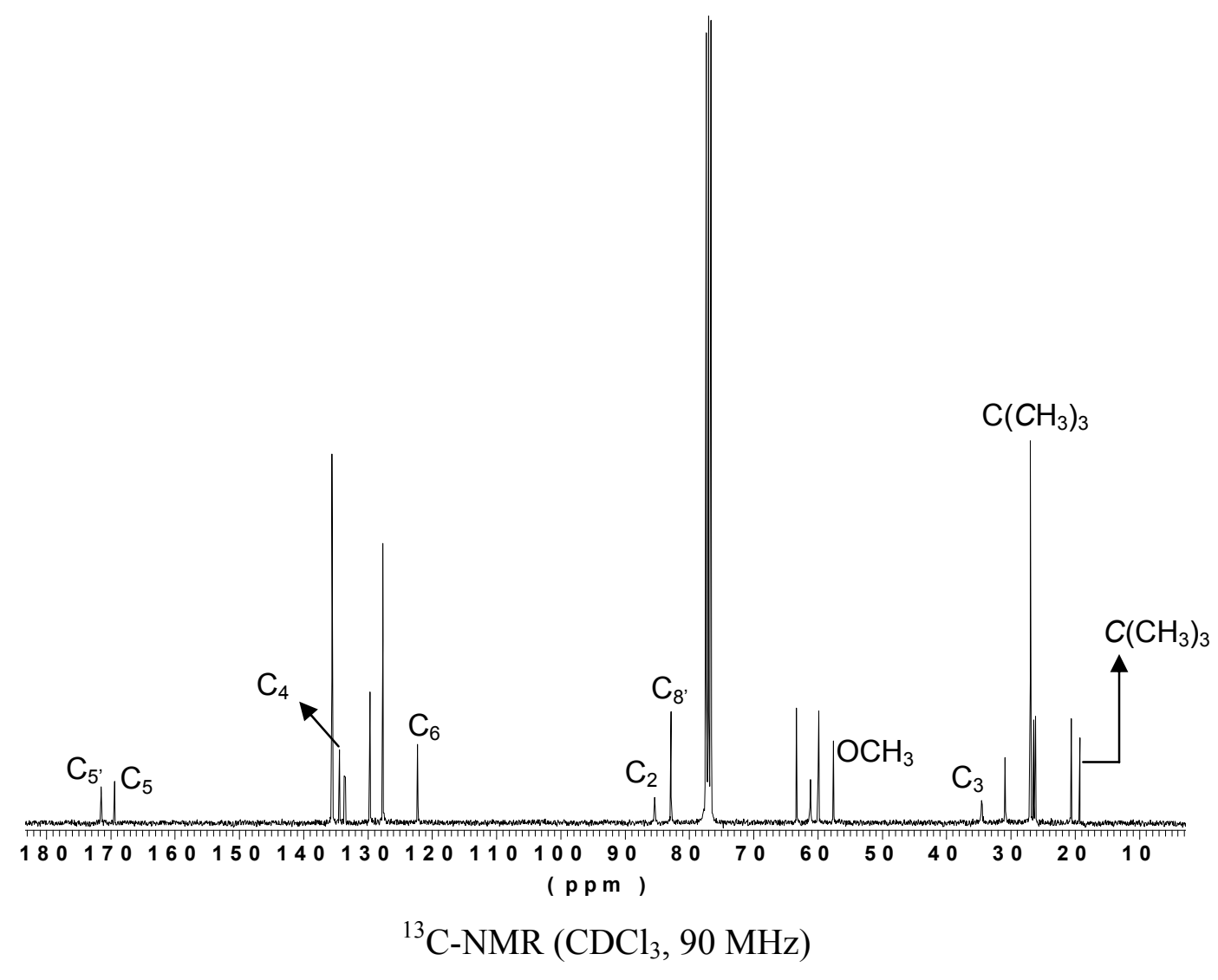




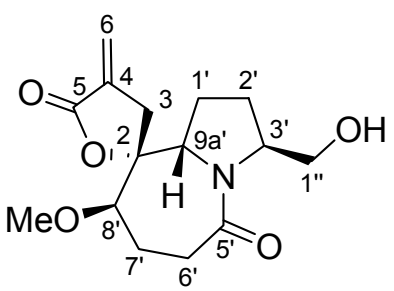

19
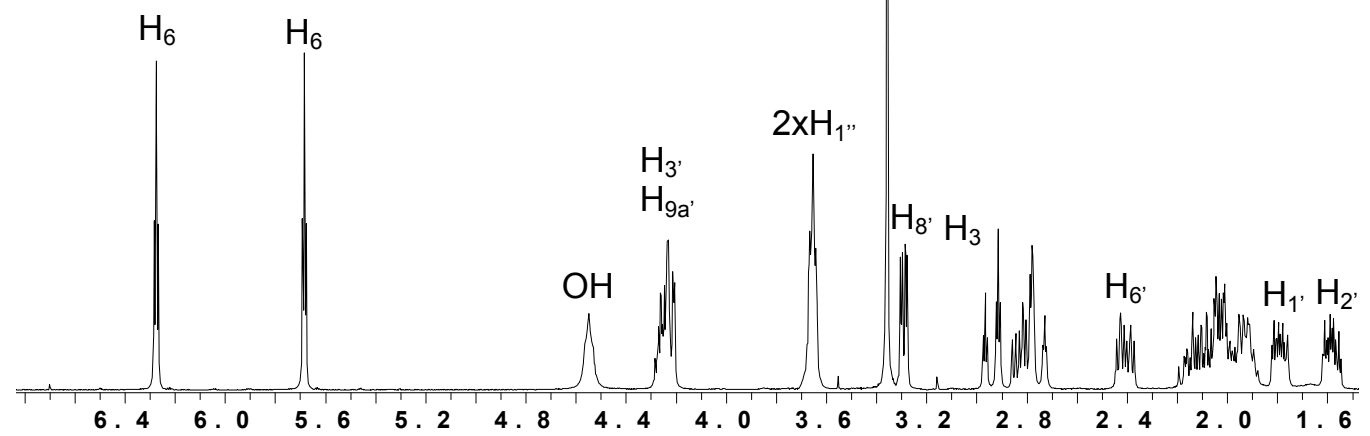

( ppm)

${ }^{1} \mathrm{H}-\mathrm{NMR}\left(\mathrm{CDCl}_{3}, 360 \mathrm{MHz}\right)$
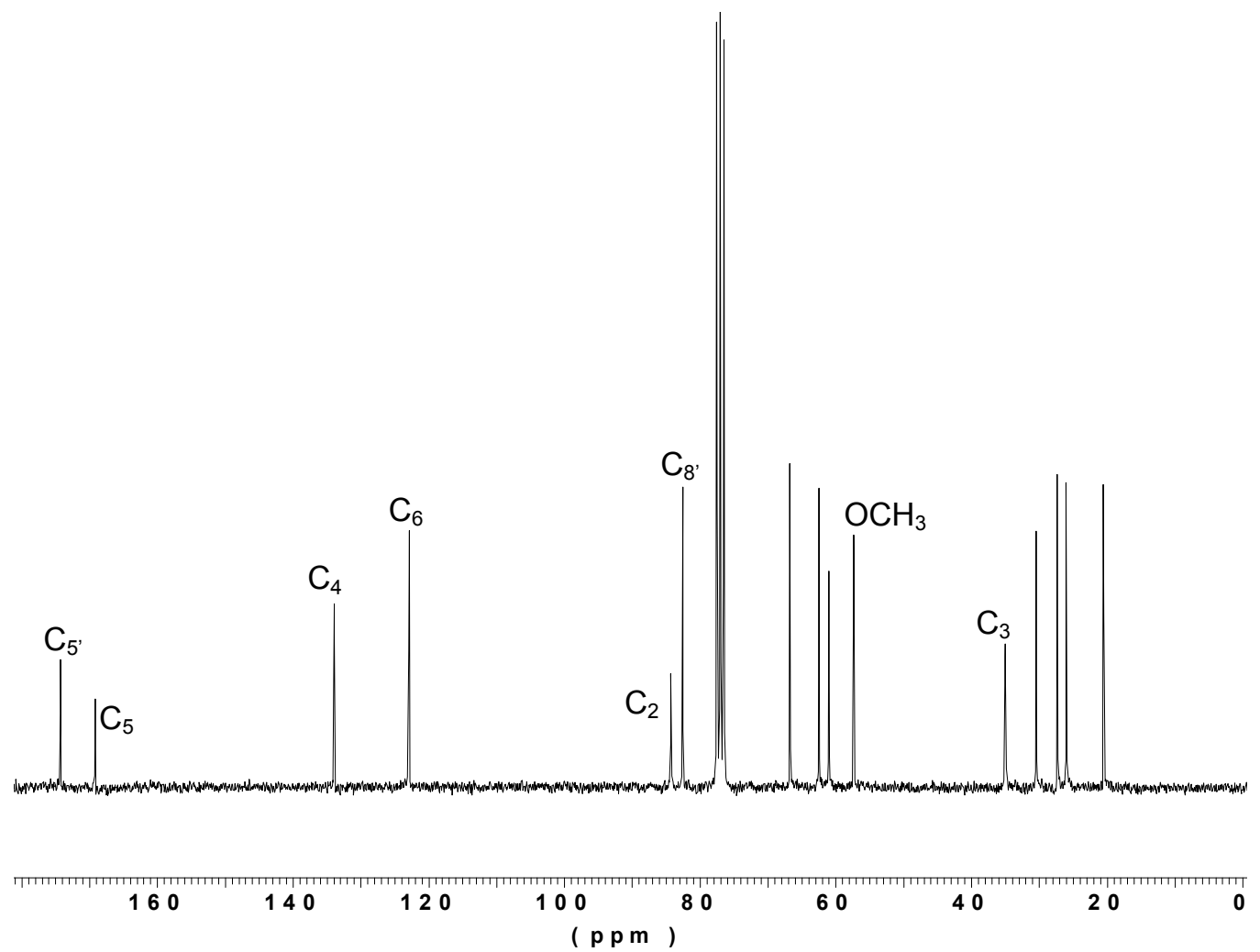

${ }^{13} \mathrm{C}-\mathrm{NMR}\left(\mathrm{CDCl}_{3}, 90 \mathrm{MHz}\right)$ 


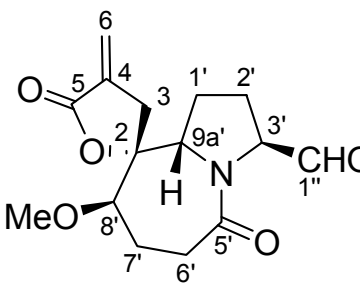

20

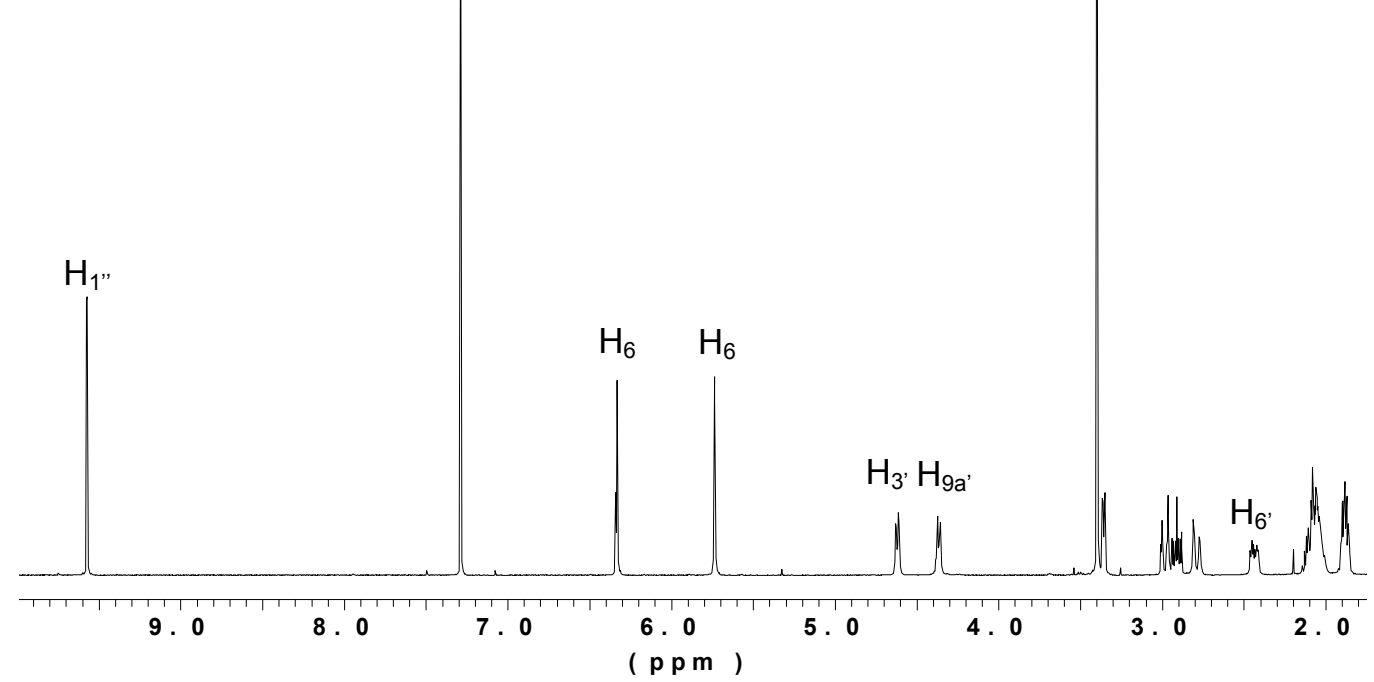

${ }^{1} \mathrm{H}-\mathrm{NMR}\left(\mathrm{CDCl}_{3}, 500 \mathrm{MHz}\right)$

$\mathrm{C}_{1}{ }^{\prime \prime}$

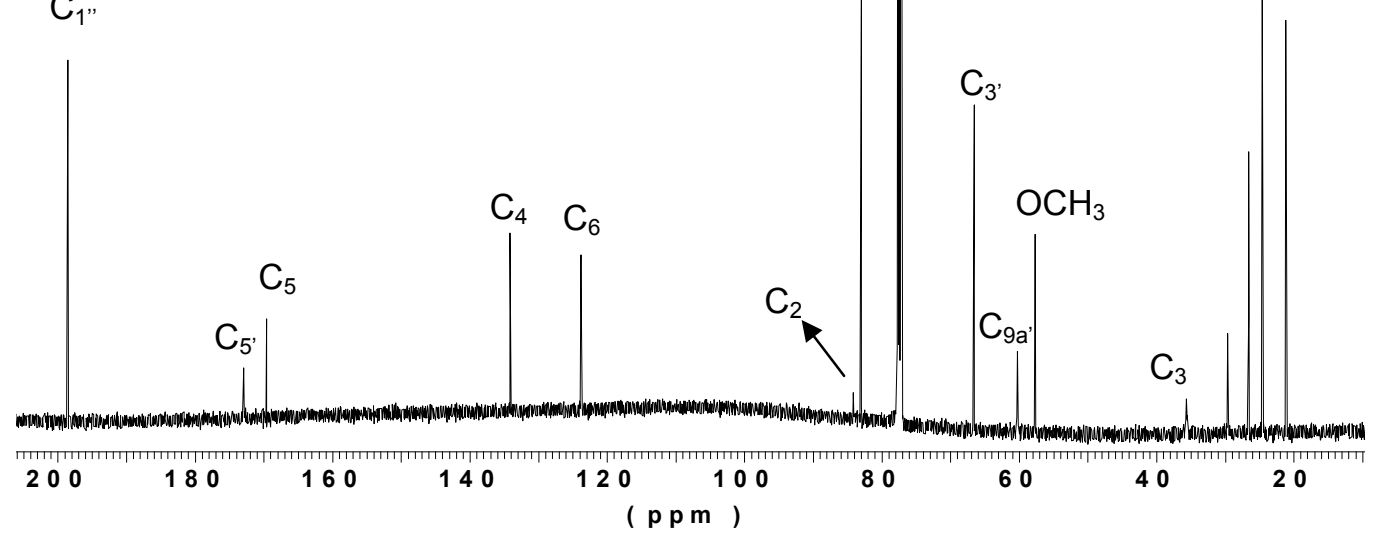

${ }^{13} \mathrm{C}-\mathrm{NMR}\left(\mathrm{CDCl}_{3}, 125 \mathrm{MHz}\right)$ 


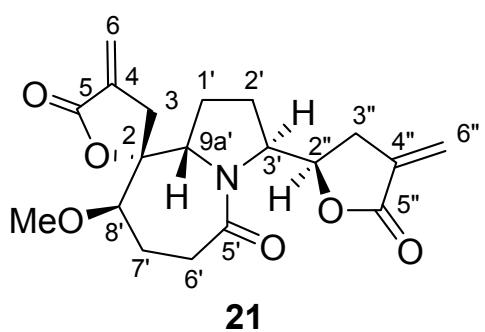

21

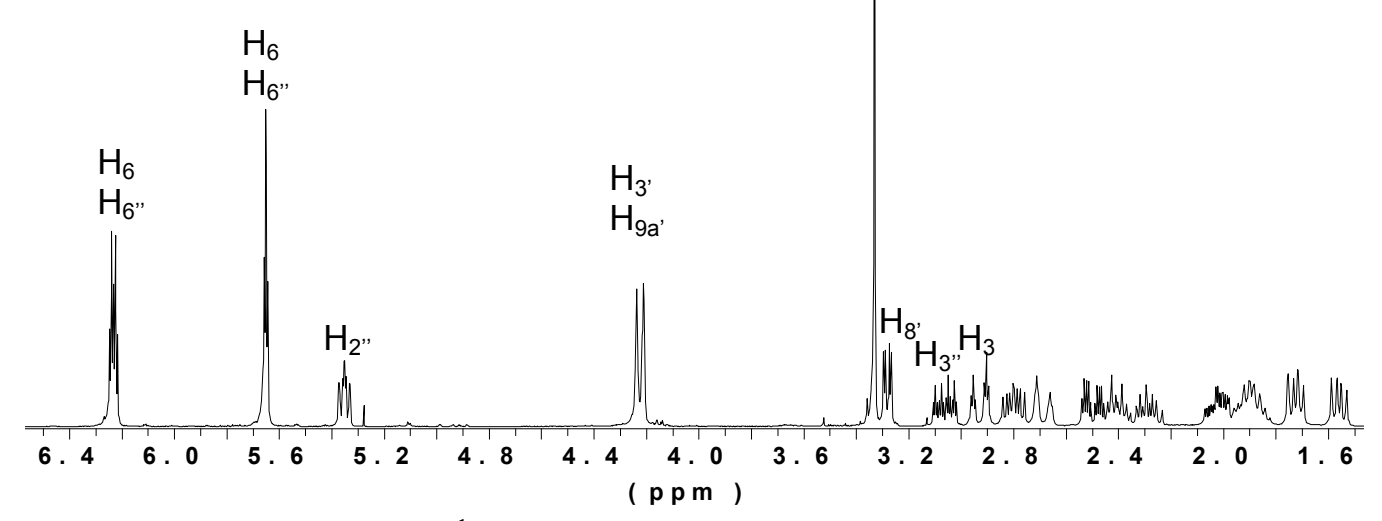

${ }^{1} \mathrm{H}-\mathrm{NMR}\left(\mathrm{CDCl}_{3}, 360 \mathrm{MHz}\right)$

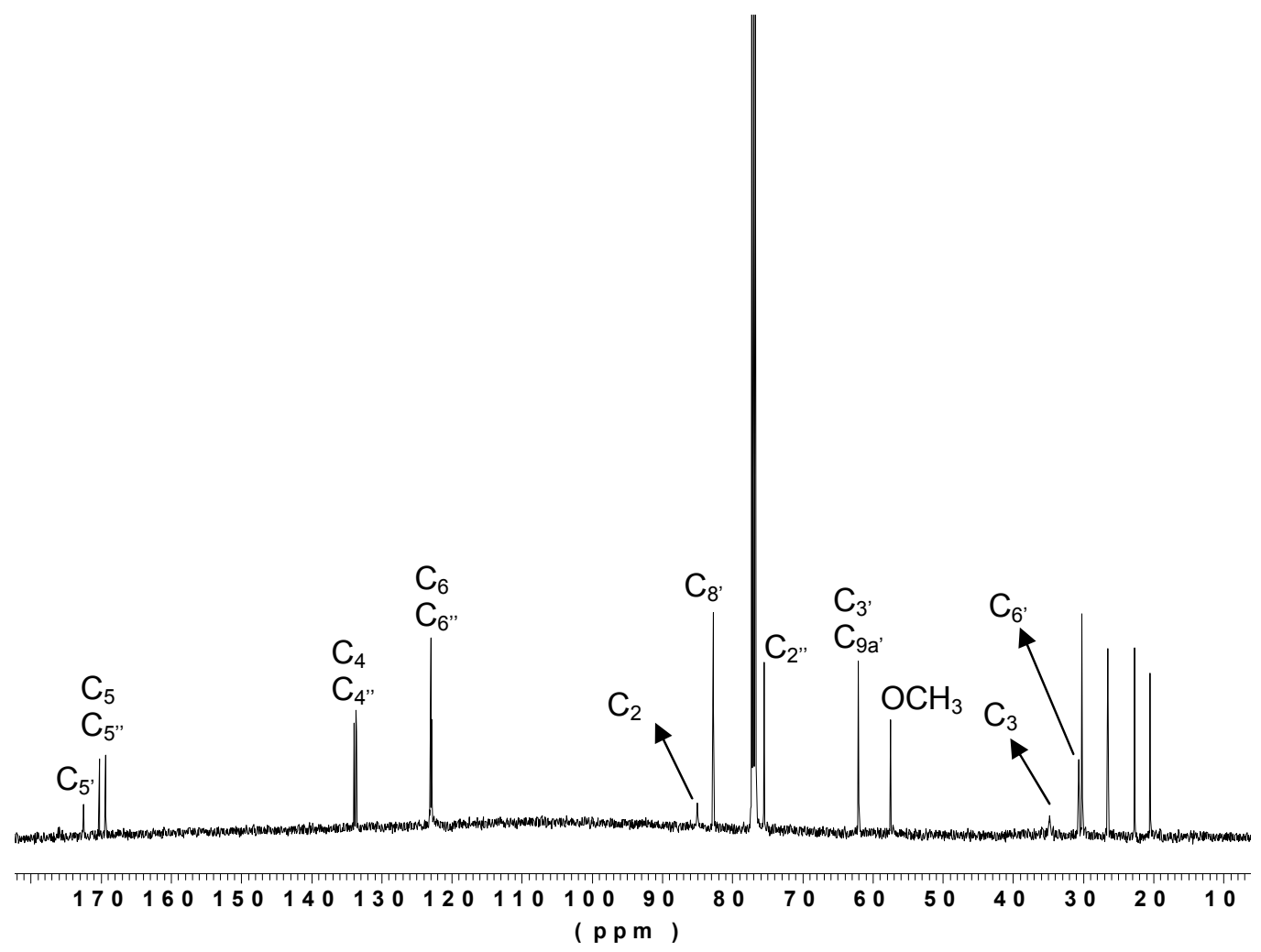

${ }^{13} \mathrm{C}-\mathrm{NMR}\left(\mathrm{CDCl}_{3}, 125 \mathrm{MHz}\right)$ 


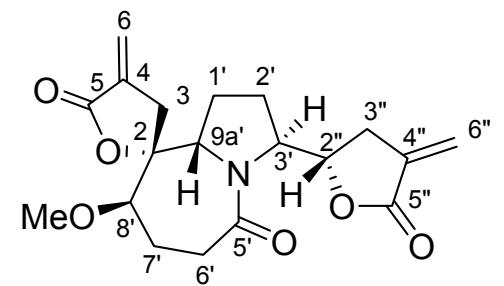

22

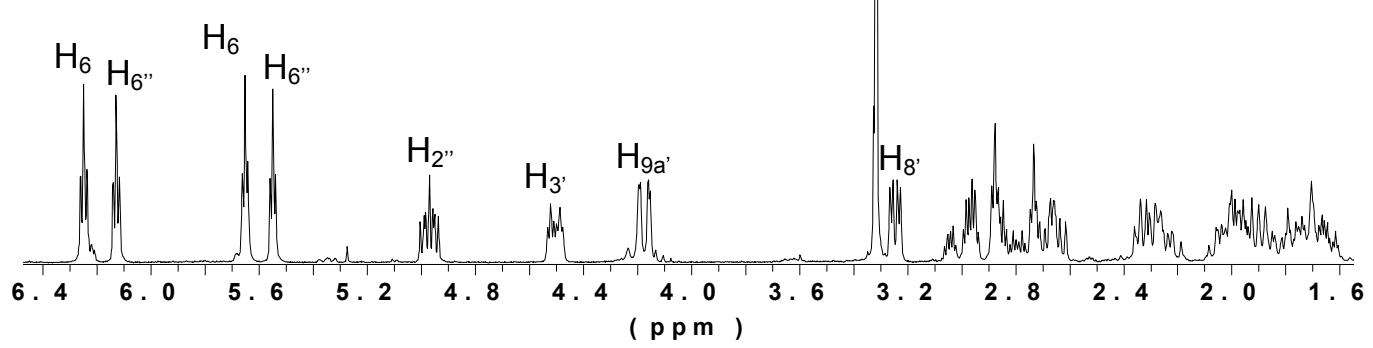

${ }^{1} \mathrm{H}-\mathrm{NMR}\left(\mathrm{CDCl}_{3}, 360 \mathrm{MHz}\right)$

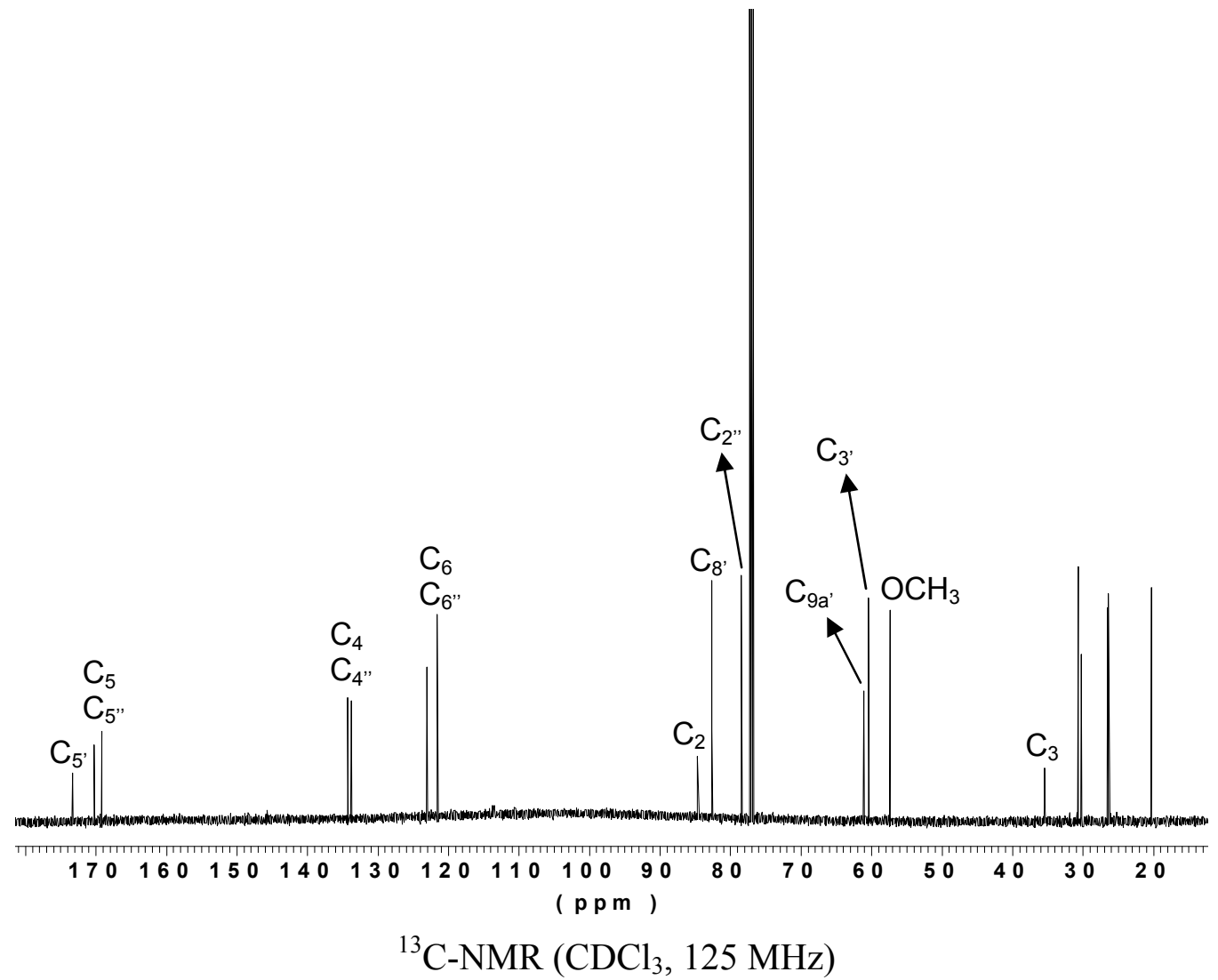




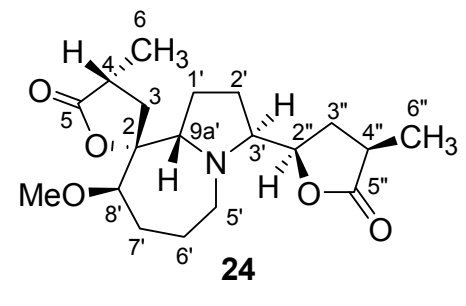

$\mid$

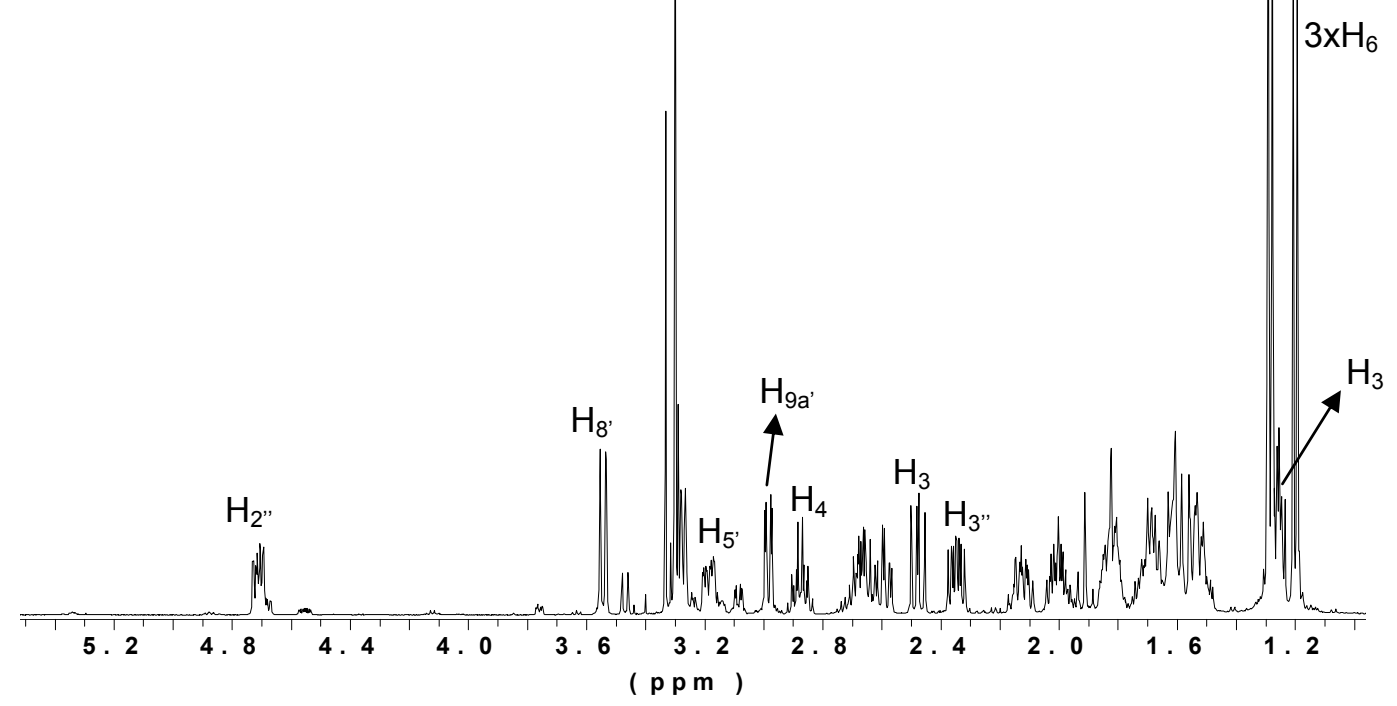

${ }^{1} \mathrm{H}-\mathrm{NMR}\left(\mathrm{CDCl}_{3}, 500 \mathrm{MHz}\right)$

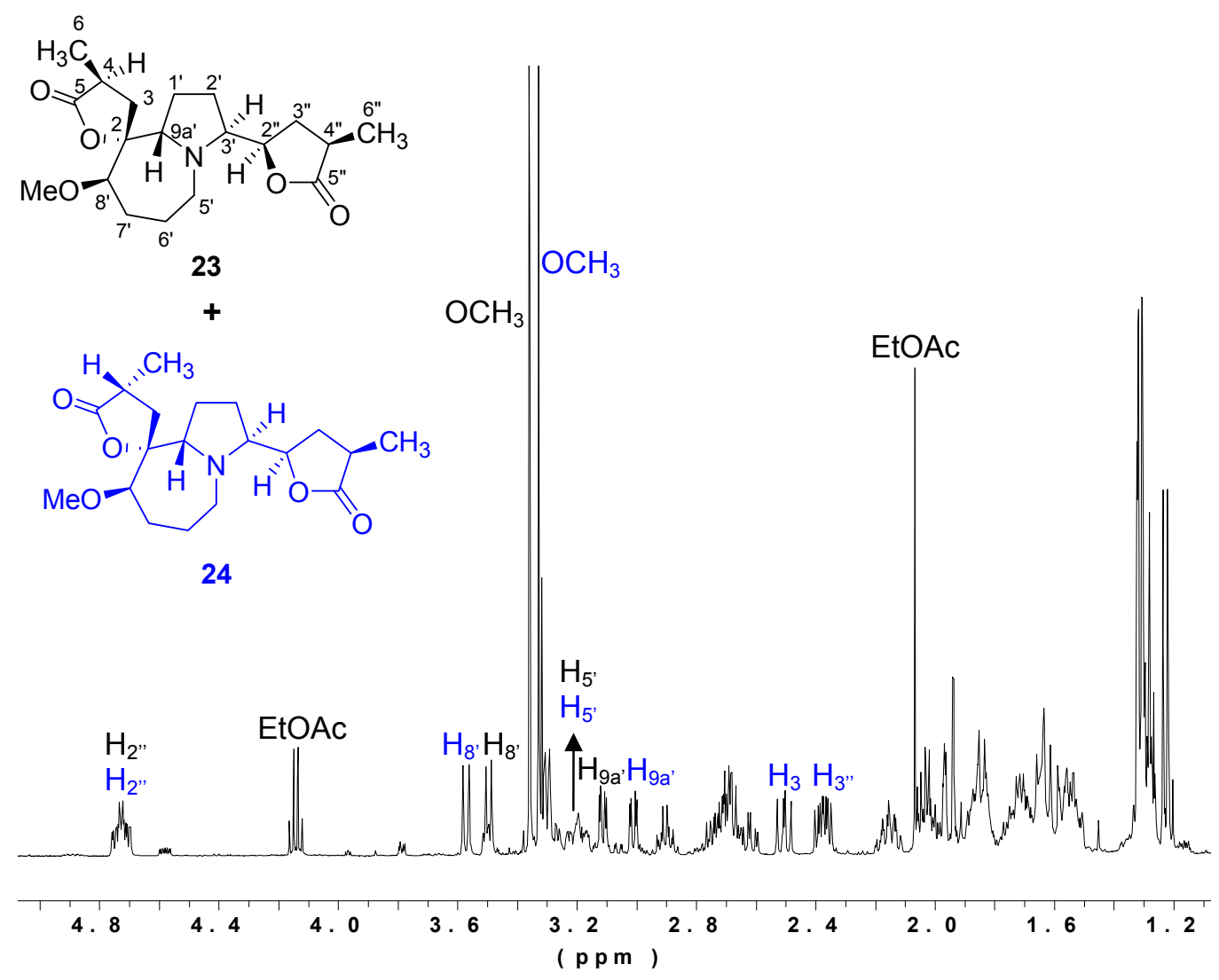

${ }^{1} \mathrm{H}-\mathrm{NMR}\left(\mathrm{CDCl}_{3}, 500 \mathrm{MHz}\right)$ 


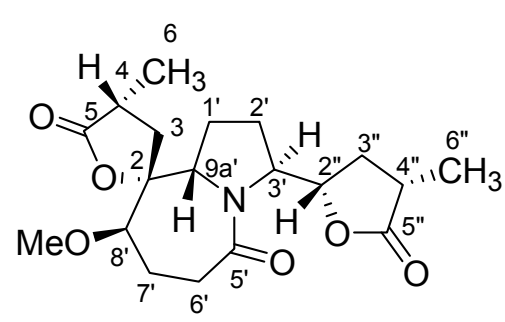

26

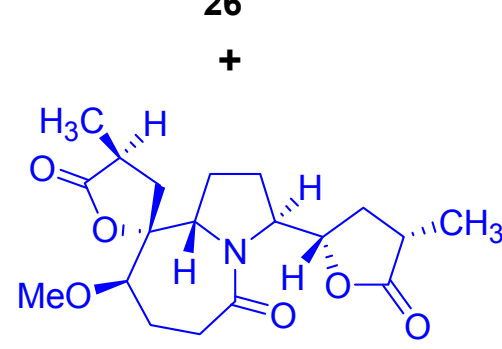

25

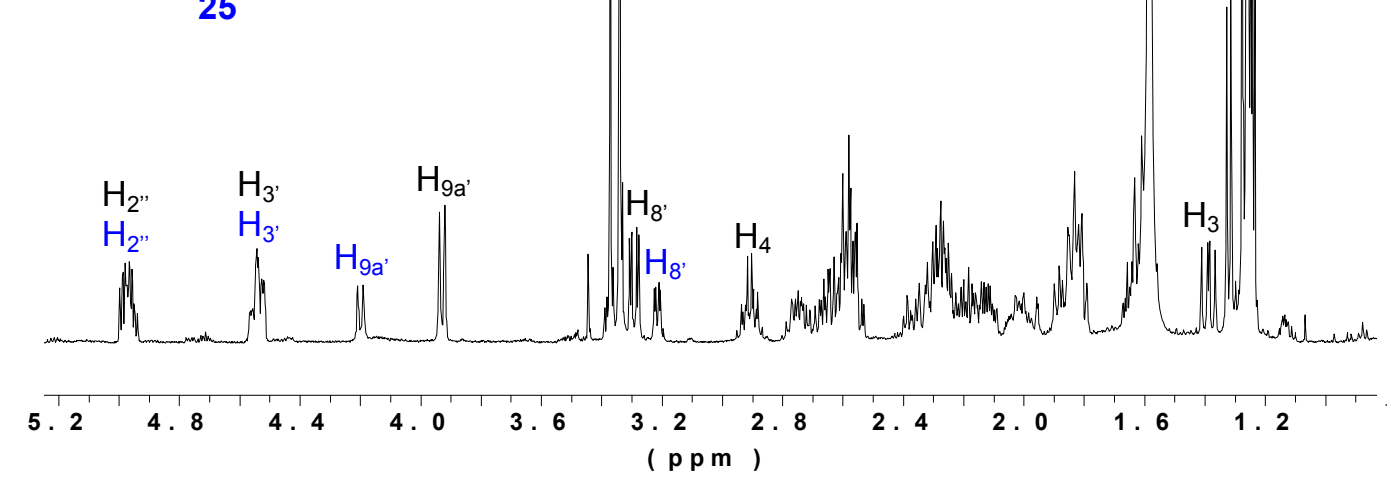

${ }^{1} \mathrm{H}-\mathrm{NMR}\left(500 \mathrm{MHz}, \mathrm{CDCl}_{3}\right)$ 

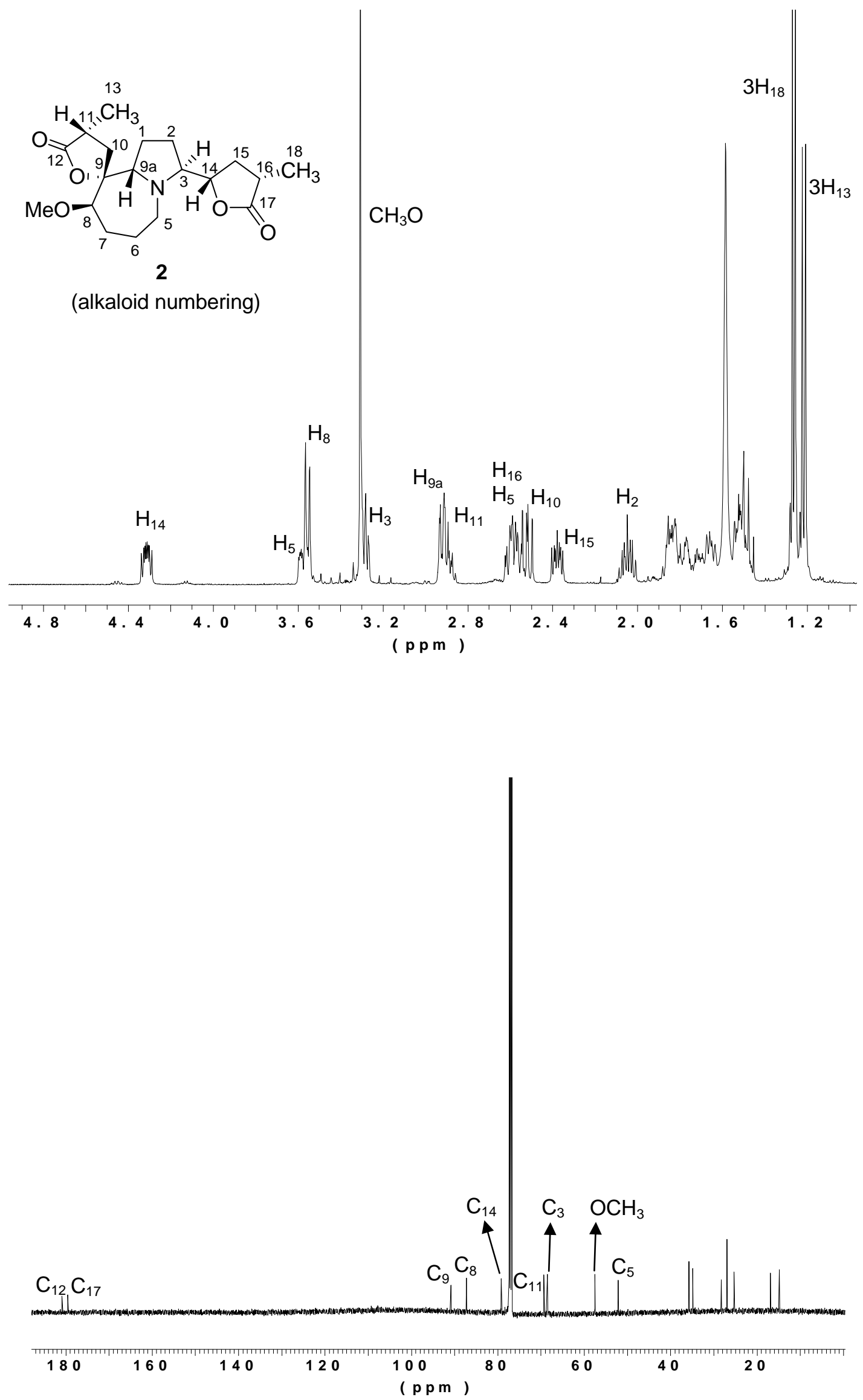

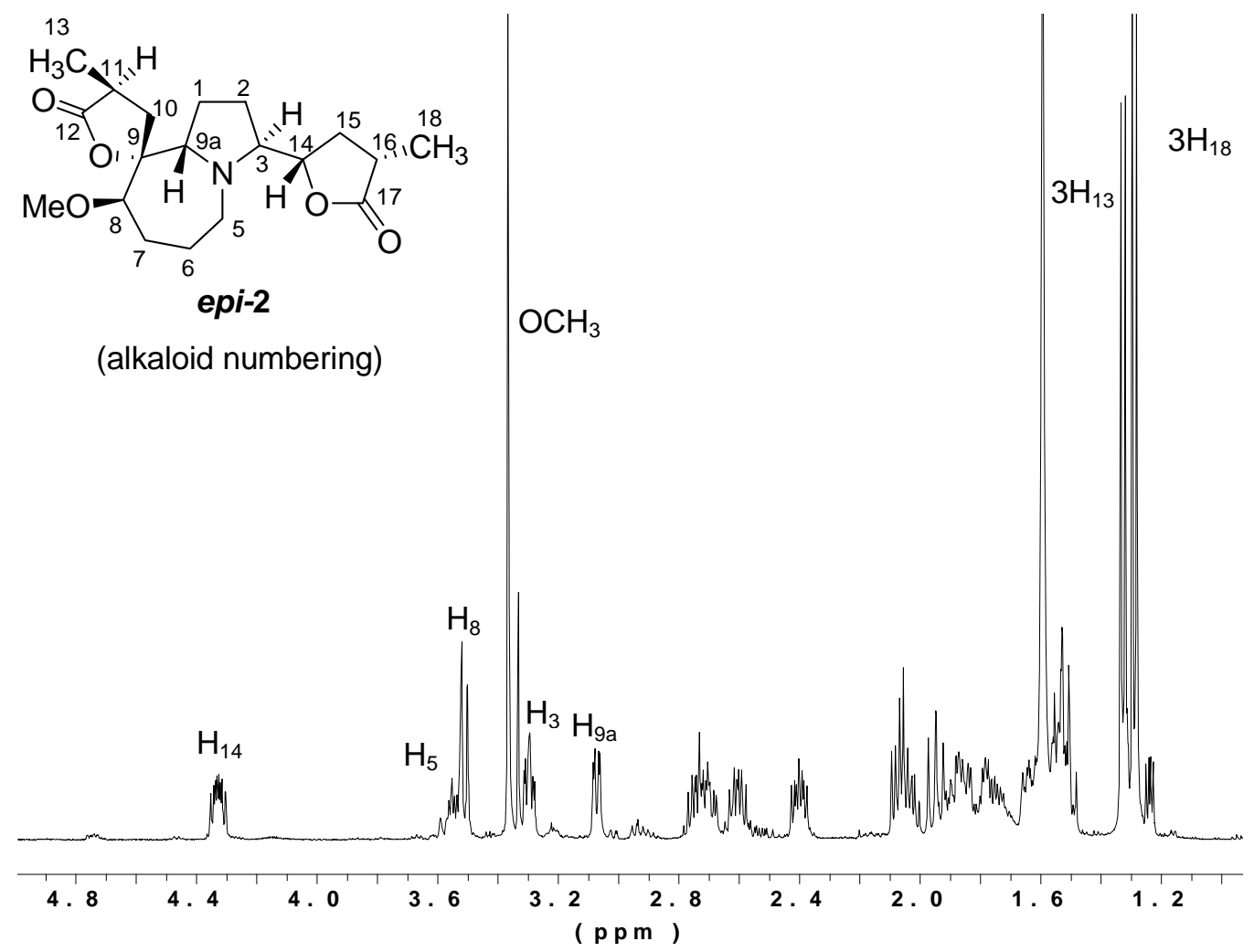

${ }^{1} \mathrm{H}-\mathrm{NMR}\left(\mathrm{CDCl}_{3}, 500 \mathrm{MHz}\right)$

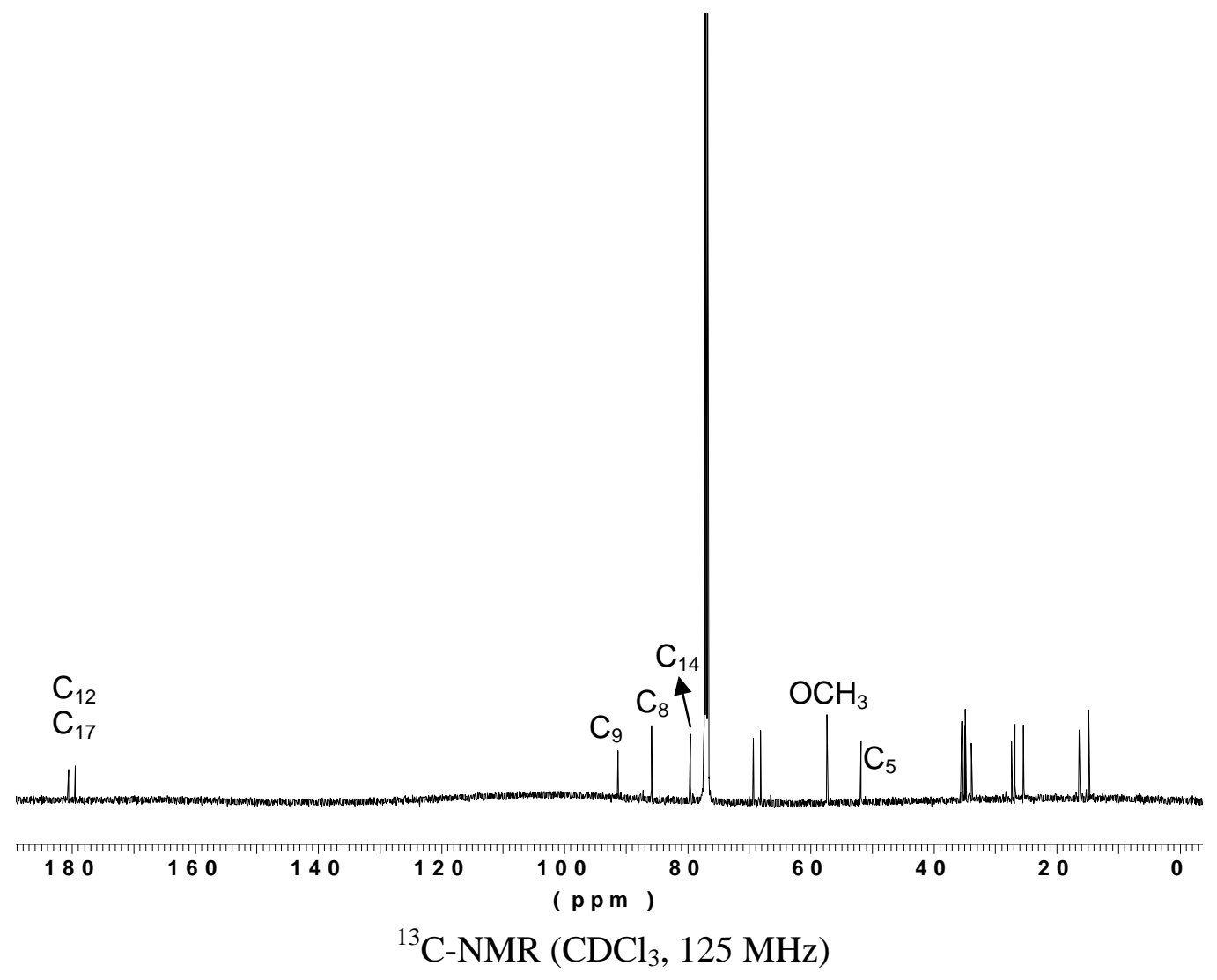

